\title{
The Average Condition Number of Most Tensor Rank Decomposition Problems is Infinite
}

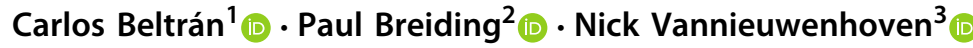

Received: 21 March 2019 / Revised: 27 April 2021 / Accepted: 23 September 2021 /

Published online: 8 February 2022

(c) The Author(s) 2022

\begin{abstract}
The tensor rank decomposition, or canonical polyadic decomposition, is the decomposition of a tensor into a sum of rank-1 tensors. The condition number of the tensor rank decomposition measures the sensitivity of the rank-1 summands with respect to structured perturbations. Those are perturbations preserving the rank of the tensor that is decomposed. On the other hand, the angular condition number measures the perturbations of the rank-1 summands up to scaling. We show for random rank- 2 tensors that the expected value of the condition number is infinite for a wide range of choices of the density. Under a mild additional assumption, we show that the same is true for most higher ranks $r \geq 3$ as well. In fact, as the dimensions of the tensor tend to infinity, asymptotically all ranks are covered by our analysis. On the contrary, we show that rank-2 tensors have finite expected angular condition number. Based on numerical experiments, we conjecture that this could also be true for higher ranks.
\end{abstract}

Communicated by Joseph M. Landsberg.

CB: Supported by Spanish "Ministerio de Economía y Competitividad" Under Project PID2020-113887GB-I00, as well as by the Banco Santander and Universidad de Cantabria Under Project 21.SI01.64658. PB: Funded by the Deutsche Forschungsgemeinschaft (DFG, German Research Foundation)_-Projektnummer 445466444. NV: Supported by the Postdoctoral Fellowship of the Research Foundation-Flanders (FWO) with Project Numbers 12E8116N and 12E8119N and partially supported by KU Leuven project STG/19/002.

$凶$ Carlos Beltrán

beltranc@unican.es

Paul Breiding

paul.breiding@mis.mpg.de

Nick Vannieuwenhoven

nick.vannieuwenhoven@kuleuven.be

1 Universidad de Cantabria, Santander, Spain

2 Max-Planck-Institute for Mathematics in the Sciences, Leipzig, Germany

3 KU Leuven, Department of Computer Science, Leuven, Belgium 
Our results underline the high computational complexity of computing tensor rank decompositions. We discuss consequences of our results for algorithm design and for testing algorithms computing tensor rank decompositions.

Keywords Tensor decomposition · Condition number · Average analysis

Mathematics Subject Classification 15A69 $\cdot$ 53B20 $\cdot 65 \mathrm{~F} 35 \cdot 14 \mathrm{Q} 20$

\section{Introduction}

\subsection{The Condition Number of Tensor Rank Decomposition}

In this article, a tensor is a multidimensional array filled with numbers:

$$
\mathcal{A}:=\left(a_{i_{1}, \ldots, i_{d}}\right)_{1 \leq i_{1} \leq n_{1}, \ldots, 1 \leq i_{d} \leq n_{d}} \in \mathbb{R}^{n_{1} \times \cdots \times n_{d}} .
$$

The integer $d$ is called the order of $\mathcal{A}$. The tensor product of $d$ vectors $\mathbf{u}^{1} \in$ $\mathbb{R}^{n_{1}}, \ldots, \mathbf{u}^{d} \in \mathbb{R}^{n_{d}}$ is defined to be the tensor $\mathbf{u}^{1} \otimes \cdots \otimes \mathbf{u}^{d} \in \mathbb{R}^{n_{1} \times \cdots \times n_{d}}$ with entries

$$
\left(\mathbf{u}^{1} \otimes \cdots \otimes \mathbf{u}^{d}\right)_{i_{1}, \ldots, i_{d}}:=u_{i_{1}}^{(1)} \cdots u_{i_{d}}^{(d)}, \text { where } \mathbf{u}^{j}=\left[u_{i}^{(j)}\right]_{1 \leq i \leq n_{j}} .
$$

Any nonzero multidimensional array obeying this relation is called a rank-1 tensor. Not every multidimensional array represents a rank-1 tensor, but every tensor $\mathcal{A}$ is a finite linear combination of rank-1 tensors:

$$
\mathcal{A}=\sum_{i=1}^{r} \mathcal{A}_{i}, \text { where } \mathcal{A}_{i}=\mathbf{u}_{i}^{1} \otimes \cdots \otimes \mathbf{u}_{i}^{d} \text { has rank one for each } 1 \leq i \leq d .
$$

Hitchcock [50] coined the name polyadic decomposition for the decomposition Eq. (1). The smallest number $r$ for which $\mathcal{A}$ admits an expression as in Eq. (1) is called the (real) rank of $\mathcal{A}$. A corresponding minimal decomposition is called a canonical polyadic decomposition (CPD).

For instance, in algebraic statistics [1,59], chemical sciences [67], machine learning [4], psychometrics [54], signal processing [35,36,65], or theoretical computer science [29], the input data have the structure of a tensor and the CPD of this tensor reveals the information of interest. Usually, this data is subject to measurement errors, which will cause the CPD computed from the measured data to differ from the CPD of the true data. In numerical analysis, the sensitivity of the model parameters, such as the rank-1 summands in the CPD, to perturbations of the data is often quantified by the condition number [61].

When there are multiple CPDs of a tensor $\mathcal{A}$, the condition number must be defined at a decomposition $\left\{\mathcal{A}_{1}, \ldots, \mathcal{A}_{r}\right\}$. However, in this article, we will restrict our analysis to tensors $\mathcal{A}$ having a unique decomposition. Such tensors are called identifiable. In

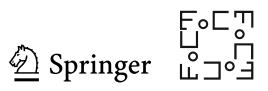


this case, the condition number of the tensor rank decomposition of a tensor $\mathcal{A}$ is welldefined, and we denote it by $\kappa(\mathcal{A})$. We will explain in Section 1.3 in greater detail the notion of identifiability of tensors. At this point, the reader should mainly bear in mind that the assumption of being identifiable is comparably weak as most tensors of low rank satisfy it. However, note that matrices $(d=2)$ are never identifiable, so we assume that the order of the tensor is $d \geq 3$.

The condition number of tensor rank decomposition was characterized in [20], and it is the condition number of the following computational problem: On input $\mathcal{A} \in$ $\mathbb{R}^{n_{1} \times \cdots \times n_{d}}$ of rank $r$, compute the set of rank-1 terms $\left\{\mathcal{A}_{1}, \ldots, \mathcal{A}_{r}\right\}$ in the decomposition Eq. (1). This condition number measures the sensitivity of the rank-1 terms with respect to perturbations of the tensor $\mathcal{A}$. In other words, when the condition number $\kappa(\mathcal{A})$ of the rank-r identifiable tensor $\mathcal{A}=\sum_{i=1}^{r} \mathcal{A}_{i}$ in Eq. (1) is finite, it is the smallest value $\kappa(\mathcal{A})$ such that

$$
\min _{\pi \in \mathfrak{S}_{r}} \sqrt{\sum_{i=1}^{r}\left\|\mathcal{A}_{i}-\mathcal{A}_{\pi_{i}}^{\prime}\right\|^{2}} \leq \kappa(\mathcal{A})\left\|\mathcal{A}-\mathcal{A}^{\prime}\right\|+o\left(\left\|\mathcal{A}-\mathcal{A}^{\prime}\right\|\right)
$$

holds for all rank-r tensors $\mathcal{A}^{\prime}=\sum_{i=1}^{r} \mathcal{A}_{i}^{\prime}$ (with $\mathcal{A}_{i}^{\prime}$ of rank 1) sufficiently close to $\mathcal{A}$. Herein, the norm on $\mathbb{R}^{n_{1} \times \cdots \times n_{d}}$ is the usual Euclidean norm, and $\mathfrak{S}_{r}$ is the permutation group on $\{1, \ldots, r\}$. It was shown in [24, Corollary 5.5] that the same expression holds if $\mathcal{A}^{\prime}$ is any tensor close to $\mathcal{A}$ and $\sum_{i=1}^{r} \mathcal{A}_{i}^{\prime}$ is the best rank-r approximation of $\mathcal{A}^{\prime}$ in the Euclidean norm.

As a general principle in numerical analysis, the condition number is an intrinsic property of the computational problem that governs the forward error and attainable precision of any method for solving the problem. Its study is also useful for other purposes. For example, in [21,22] the local rate of convergence of Riemannian GaussNewton optimization methods for computing the CPD was related to the condition number $\kappa(\mathcal{A})$.

A conventional wisdom in numerical analysis is that it is harder to compute the condition number of a given problem instance than solving the problem itself [38, 39]. This viewpoint led Smale to initiate the study of the probability distribution of condition numbers: If the condition number is small with high probability, then for many practical purposes one can assume that any given input is well-conditioned; at least the probability of failure necessarily will be small. Smale started studying the probability that a polynomial is ill-conditioned [66]. This strategy was extended to linear algebra condition numbers [26,31,41], to systems of polynomial equations in diverse settings [42,62], to linear systems of inequalities [49], to linear and convex programming $[2,68]$, eigenvalue and eigenvectors in the classic and other settings [7], to polynomial eigenvalue problems [6,8], and to other computational models [30], among others. As there is a substantive bibliography on this setting, we refer the reader to [28] for further details.

Tensor rank decomposition seems to be no exception to this wisdom: The characterization of $\kappa(\mathcal{A})$ for a given $\mathcal{A}$ in [20] requires the CPD of $\mathcal{A}$ itself. This forces us to rely on probabilistic studies to establish reasonable a priori values of the condition number. Settling this is the main purpose of this paper.

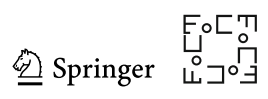




\subsection{Informal Version of Our Main Results and Discussion}

The first probabilistic analyses of the condition number of CPD were given in [11,23]. In those references, the expected value was computed for random rank-1 tensors; that is, for random output of the computational problem of computing CPDs. This amounts to choosing random $\mathbf{u}_{i}^{k}$ in the notation above, constructing the corresponding tensor $\mathcal{A}$ and studying $\kappa(\mathcal{A})$. The probabilistic study is feasible, in principle, because one can obtain a closed expression for $\kappa(\mathcal{A})$ which is polynomial in terms of the $\mathbf{u}_{i}^{k}$, so that the question boils down to an explicit but nontrivial integration problem.

This article is the first to investigate the condition number for random input. That is, we assume that $\mathcal{A}$ is chosen at random within the set of rank- $r$ tensors (see the definition of random tensors in Definition 1 and the extension in Theorem 4) and we wonder about the expected value of $\kappa(\mathcal{A})$. The difficulty now is that even if we assume that a decomposition (1) exists, we do not have it and hence we lack a closed expression for $\kappa(\mathcal{A})$.

One may wonder if these two different random procedures should give similar distributions in this or other numerical problems. The answer is no. For example, say that our problem is to compute the kernel of a given matrix $A \in \mathbb{R}^{n \times(n+1)}$ and we want to study the expected value of the associated condition number $\|A\|\left\|A^{\dagger}\right\|$. Choosing $A$ at random produces $\mathbb{E}\left(\|A\|\left\|A^{\dagger}\right\|\right)<\infty$ but choosing the kernel at random and then $A$ at random within the matrices with that kernel is the same as computing the expected value of the usual Turing's condition number of a square real Gaussian matrix, which is infinity; see [31] for precise estimations of these quantities. The situation is similar in the study of systems of homogeneous polynomial equations: random inputs have better condition number than inputs produced from random outputs; see for example [9]. In both these examples, the condition number of input constructed from random output is, on average, larger than the condition number of random input. This is a stroke of luck since in general one expects instances from practical, real-life problems, to be somehow random within the input space, not to have a random output!

In this paper, we show that computing the CPD is a rara avis: we prove in Theorems 1 and 2 that (under suitable hypotheses) the condition number of random input tensors turns out to be infinity. On the contrary, by Beltrán et al. [11] and Breiding and Vannieuwenhoven [23] it is presumed that the average condition number is finite when choosing random output. This result reinforces the evidence that computing CPDs is a very challenging computational problem.

The literature often cites the result of Håstad [53] to underline the high computational complexity of computing CPDs. Håstad showed that the NP-complete 3-satisfiability problem (also called 3-SAT) can be reduced to computing the rank of a tensor; hence, solving the tensor rank decomposition problem is NP-complete in the Turing machine computational model. Our main result is different in two aspects: First, Håstad showed the difficulty of only one particular instance of a CPD, whereas we show that computing the CPD is difficult on average. Second, our evidence supporting the hardness of the problem is not based on Turing machine complexity, but given by analyzing the condition number, which is more appropriate for numerical computations [16]. Linking complexity analyses to condition numbers is common in

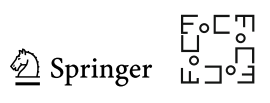


the literature; for instance, in the case of solving polynomial systems $[9,27,56,63]$. In general, the book [28] provides a good overview. In this interpretation, we show that computing CPDs numerically is hard on average.

On the other hand, in the literature, the main result of de Silva and Lim [37] is often cited as a key reason why approximating a tensor by a low-rank CPD is such a challenging problem: for some input tensors, a best low-rank approximation may not exist! This is because the set of tensors of bounded rank is not closed: There are tensors of rank strictly greater than $r$ that can be approximated arbitrarily well by rank$r$ tensors. It is shown in [37] that this ill-posedness of the approximation problem is not rare in the sense that for every tensor space $\mathbb{R}^{n_{1} \times n_{2} \times n_{3}}$ there exists an open set of input tensors which do not admit a best rank-2 approximation. This result is stronger than Håstad's in the sense that it proves that instances with no solution to the tensor rank approximation problem may occur on an open set, rather than in one particular set of measure zero. Notwithstanding this key result, it does not tell us about the complexity of solving the tensor rank decomposition problem, in which we are given a rank-r tensor whose CPD we seek. In this setting, there are no ill-posed inputs in the sense of de Silva and Lim [37]. It was already shown in [20] that the condition number diverges as one moves toward the open part of the boundary of tensors of bounded rank, entailing that there exist regions with arbitrarily high condition number. One of the main result of this paper, Theorem 2, shows that such regions cannot be ignored: They are sufficiently large to cause the integral of the condition number over the set of rank- $r$ tensors to diverge. In other words, one cannot neglect the regions where the condition number is so high that a CPD computed from a floating-point representation of a rank- $r$ tensor in $\mathbb{R}^{n_{1} \times \cdots \times n_{d}}$, subject only to roundoff errors, is meaningless-a result similar in spirit to de Silva and Lim [37].

One may conclude from the above that, at least from the point of view of average stability of the problem, tensor rank decomposition is doomed to fail. However, if one only cares about the directions of the rank-1 terms in the decomposition, then the situation changes dramatically. The condition number associated with the computational problem "Given a rank-r identifiable tensor $\mathcal{A}=\sum_{i=1}^{r} \mathcal{A}_{i}$ as in Eq. (1), output the set of normalized rank-1 tensors $\left\{\frac{\mathcal{A}_{i}}{\left\|\mathcal{A}_{1}\right\|}, \ldots, \frac{\mathcal{A}_{r}}{\left\|\mathcal{A}_{r}\right\|}\right\}$ " will be called the angular condition number $\kappa_{\text {ang }}(\mathcal{A})$. Analogously to the bound Eq. (2), one can show that when $\kappa_{\text {ang }}$ is finite, it is the smallest number such that

$$
\min _{\pi \in \mathfrak{S}_{r}} \sqrt{\sum_{i=1}^{r}\left\|\frac{\mathcal{A}_{i}}{\left\|\mathcal{A}_{i}\right\|}-\frac{\mathcal{A}_{\pi_{i}}^{\prime}}{\left\|\mathcal{A}_{\pi_{i}}^{\prime}\right\|}\right\|^{2}} \leq \kappa_{\mathrm{ang}}(\mathcal{A})\left\|\mathcal{A}-\mathcal{A}^{\prime}\right\|+o\left(\left\|\mathcal{A}-\mathcal{A}^{\prime}\right\|\right)
$$

for all rank-r tensors $\mathcal{A}^{\prime}=\sum_{i=1}^{r} \mathcal{A}_{i}^{\prime}$ (with $\mathcal{A}_{i}^{\prime}$ rank-1 tensors) in a sufficiently small open neighborhood of $\mathcal{A}$. By Breiding and Vannieuwenhoven [24, Corollary 5.5], the same expression holds for all tensors $\mathcal{A}^{\prime}$ in a small open neighborhood of $\mathcal{A}$ if $\sum_{i=1}^{r} \mathcal{A}_{i}^{\prime}$ is the best rank-r approximation of $\mathcal{A}^{\prime}$.

We will prove in Theorem 3 that at least in the case of rank- 2 tensors, the angular condition number $\kappa_{\text {ang }}$ for random inputs is finite, contrary to the classic condition number $\kappa$; in fact, the numerical experiments in Sect. 7 suggest that this finite average condition seems to extend to much higher ranks as well. In other words, on average 
we may expect to be able to recover the angular part of the CPD:

$$
\mathcal{U}_{i}=\frac{\mathcal{A}_{i}}{\left\|\mathcal{A}_{i}\right\|}, \quad \text { for } i=1, \ldots, r
$$

where $\mathcal{A}_{i}$ is as in Eq. (1). One could conclude from this that a tensor decomposition algorithm should aim to produce the normalized rank-1 terms $\mathcal{U}_{i}$ from the tensor rank decomposition

$$
\mathcal{A}=\sum_{i=1}^{r} \lambda_{i} \mathcal{U}_{i}
$$

accurately. Once these terms are obtained, one can recover the $\lambda_{i}$ 's by solving a linear system of equations. Since, as a general principle, the condition number of a composite smooth map $g \circ f$ between manifolds satisfies [16,28]

$$
\kappa[g \circ f](x):=\left\|\left(\mathrm{d}_{f(x)} g\right)\left(\mathrm{d}_{x} f\right)\right\| \leq\left\|\mathrm{d}_{f(x)} g\right\|\left\|\mathrm{d}_{x} f\right\|=\kappa[g](f(x)) \kappa[f](x),
$$

it follows that the condition number of tensor decomposition is bounded by the product of the condition numbers of the problem of finding the angular part of the CPD and the condition number of solving a linear least-squares problem. Our main results suggest that precisely the last problem will on average be ill-conditioned.

The foregoing observation can have major implications for algorithm design. Indeed, solving the tensor rank decomposition problem by first solving for the angular part and then the linear least-squares problem decomposes the problem into a nonlinear and a linear part. Crucially, the latter least-squares problem can be solved by direct methods, such as a QR-factorization combined with a linear system solver. Such methods have a uniform computational cost regardless of the condition number of the problem. By contrast, since no (provably) numerically stable direct algorithms for tensor rank decomposition are currently known Beltrán et al. [11], iterative methods are indispensable for this problem. We may expect their computational performance to depend on the condition number of the problem instance. Indeed, our main results combined with the main result of Breiding and Vannieuwenhoven [21] imply, for example, that Riemannian Gauss-Newton optimization methods for solving the angular part of the CPD should, on average, require less iterations to reach convergence than Riemannian Gauss-Newton methods for solving the tensor decomposition problem directly (such as the methods in [21,22]), because the angular condition number $\kappa$ ang appears to be finite on average, while the regular condition number $\kappa$ is proved to be $\infty$ on average in most cases, as we show in this article.

Our main results also have consequences for researchers testing numerical algorithms for computing the CPD. In the literature, a common way of generating input data for testing algorithms is to sample the rank-1 terms $\mathcal{A}_{i}=\lambda_{i} \mathbf{u}_{i}^{1} \otimes \mathbf{u}_{i}^{2} \otimes \cdots \otimes \mathbf{u}_{i}^{d}$ randomly, and then apply the algorithm to the associated tensor $\mathcal{A}=\sum_{i=1}^{r} \mathcal{A}_{i}$. However, our analysis in this paper and the analyses in $[11,23]$ show that this procedure generates tensors that are heavily biased toward being numerically well-conditioned. Hence, this way of testing algorithms probably does not correspond to a realistic distribution

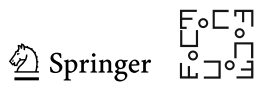


on the inputs. We acknowledge that it is currently not easy to sample rank- $r$ tensors uniformly even though some methods exist [18]. In part, this is because equations for the algebraic variety containing the tensors of rank bounded by $r$ are hard to obtain [57]. Nevertheless, in Sect. 7, using the observation from Remark 2, we present an acceptance-rejection method that can be applied to a few cases and yields uniformly distributed rank- $r$ tensors, relative to the Gaussian density in Definition 1 . In any case we strongly advocate that the (range of) condition numbers are reported when testing the performance of iterative methods for solving the tensor rank decomposition problem, so that one can assess the difficulty of the problem instances. We believe it is always recommended to include models that are known to lead to instances with high condition numbers, such as those used in [20,22].

The formal presentation of our main results requires some extra notation that we introduce in subsequent sections.

\subsection{Identifiable Tensors and a Formula for the Condition Number}

A particular feature of higher-order tensors that distinguishes them from matrices is identifiability. This means that in many cases the CPD of tensors of order $d \geq 3$ of small rank is unique. A tensor $\mathcal{A} \in \mathbb{R}^{n_{1} \times \cdots \times n_{d}}$ is called $r$-identifiable if there is a unique set $\left\{\mathcal{A}_{1}, \ldots, \mathcal{A}_{r}\right\}$ of cardinality $r$ such that $\mathcal{A}=\mathcal{A}_{1}+\cdots+\mathcal{A}_{r}$ and all $\mathcal{A}_{i}$ 's are rank-1 tensors. A celebrated criterion by Kruskal [55] gives a tool to decide if a given tensor of order 3 satisfies this property.

Lemma 1 (Kruskal's criterion [55,64]) Let $\mathbb{F}$ be $\mathbb{R}$ or $\mathbb{C}, \mathcal{A} \in \mathbb{F}^{n_{1} \times n_{2} \times n_{3}}$ a tensor of order 3 and assume that $\mathcal{A}=\sum_{i=1}^{r} \mathcal{A}_{i}$, where $\mathcal{A}_{i}=\lambda_{i} \mathbf{u}_{i}^{1} \otimes \mathbf{u}_{i}^{2} \otimes \mathbf{u}_{i}^{3} \in \mathbb{F}^{n_{1} \times n_{2} \times n_{3}}$. Define the factor matrices $U_{\ell}=\left[\mathbf{u}_{i}^{\ell}\right]_{1 \leq i \leq r} \in \mathbb{F}^{n_{\ell} \times r}$ for $\ell=1,2,3$, and let $k_{\ell}$ be the largest integer $k$ such that every subset of $k$ columns of $U_{\ell}$ has rank equal to $k$. If $r \leq \frac{1}{2}\left(k_{1}+k_{2}+k_{3}-2\right)$ and $k_{1}, k_{2}, k_{3}>1$, then the tensor $\mathcal{A}$ is $r$-identifiable over $\mathbb{F}$.

Since matrix rank does not change with a field extension from $\mathbb{R}$ to $\mathbb{C}$, a real rank- $r$ tensor $\mathcal{A} \in \mathbb{R}^{n_{1} \times n_{2} \times n_{3}}$ that satisfies the assumptions of Lemma 1 is $r$-identifiable over $\mathbb{R}$ and also automatically $r$-identifiable over $\mathbb{C}$. In other words, Kruskal's criterion is certifying complex $r$-identifiability of tensors, which is a strictly stronger notion than $r$-identifiability over $\mathbb{R}[5]$.

Most order 3 tensors of low-rank satisfy Kruskal's criterion [34]: There is an open dense subset of the set of rank- $r$ tensors in $\mathbb{R}^{n_{1} \times n_{2} \times n_{3}}, n_{1} \geq n_{2} \geq n_{3} \geq 2$, where complex $r$-identifiability holds, provided $r \leq n_{1}+\min \left\{\frac{1}{2} \delta, \delta\right\}$ with $\delta:=n_{2}+n_{3}-$ $n_{1}-2$. In fact, this phenomenon occurs much more generally than third-order tensors of very small rank. Let us denote the set of complex tensors of complex rank bounded by $r$ by

$$
\sigma_{r ; n_{1}, \ldots, n_{d}}^{\mathbb{C}}:=\left\{\mathcal{A} \in \mathbb{C}^{n_{1} \times \cdots \times n_{d}} \mid \operatorname{rank}_{\mathbb{C}}(\mathcal{A}) \leq r\right\} .
$$

This constructible ${ }^{1}$ set turns out to be an open dense subset (in the Euclidean topology) of its Zariski closure $\overline{\sigma_{r ; n_{1}, \ldots, n_{d}}^{\mathbb{C}}}$; see [57]. One says that $\sigma_{r ; n_{1}, \ldots, n_{d}}^{\mathbb{C}}$ is generically

\footnotetext{
$\overline{1}$ The elements of $\sigma_{r ; n_{1}, \ldots, n_{d}}^{\mathbb{C}}$ can be parameterized as in Eq. (1) changing $\mathbb{R}$ to $\mathbb{C}$.
} 
complex $r$-identifiable if the subset of points of $\sigma_{r ; n_{1}, \ldots, n_{d}}^{\mathbb{C}}$ that are not complex $r$ identifiable is contained in a proper closed subset in the Zariski topology on the algebraic variety $\overline{\sigma_{r ; n_{1}, \ldots, n_{d}}^{\mathbb{C}}}$; see [32]. It is known from dimensionality arguments [32] that there is a maximum value of $r$ for which generic $r$-identifiability of $\sigma_{r ; n_{1}, \ldots, n_{d}}$ can hold, namely

$$
r \leq r_{n_{1}, \ldots, n_{d}}^{\mathrm{crit}}, \quad \text { where } \quad r_{n_{1}, \ldots, n_{d}}^{\mathrm{crit}}:=\frac{n_{1} \cdots n_{d}}{1+\sum_{k=1}^{d}\left(n_{k}-1\right)}
$$

In fact, it is conjectured that the inequality is strict in general; see [47] for details. For all other values of $r$, generic $r$-identifiability does not hold. In $[17,32,33,40]$, it is proved that in the majority of choices for $n_{1}, \ldots, n_{d}$, generic complex $r$-identifiability holds for most ranks with $r<r_{\text {crit }}$; see [17, Theorem 7.2] for a result that is asymptotically optimal. For a summary of the conjecturally complete picture of complex $r$-identifiability results, see [34, Section 3].

Assumption 1 In the rest of this article, we will assume that $\sigma_{r ; n_{1}, \ldots, n_{r}}^{\mathbb{C}}$ is generically complex $r$-identifiable.

The reason why we make this assumption is because it greatly simplifies some of the arguments. At the same time, Assumption 1 is (conjectured to be) extremely weak and only limits the generality in the exceptional cases listed in [33, Theorem 1.1], and even then generic $r$-identifiability only fails very close to the upper bound $r_{\text {crit }}$ of the permitted ranks.

An immediate benefit of Assumption 1 is that it allows for a nice expression of the condition number of the tensor rank decomposition problem. Let us denote the set of rank-1 tensors in $\mathbb{R}^{n_{1} \times \cdots \times n_{d}}$ by

$$
\mathcal{S}_{n_{1}, \ldots, n_{d}}=\left\{\mathbf{a}^{1} \otimes \cdots \otimes \mathbf{a}^{d} \mid \mathbf{a}^{k} \in \mathbb{R}^{n_{k}} \backslash\{0\}\right\}
$$

It is a smooth manifold, called the Segre manifold [46,57]. The set of tensors of rank bounded by $r$ is the image of the addition map: $\sigma_{r ; n_{1}, \ldots, n_{d}}=\Phi\left(\mathcal{S}_{n_{1}, \ldots, n_{d}}^{\times r}\right)$, where

$$
\Phi: \mathcal{S}_{n_{1}, \ldots, n_{d}} \times \cdots \times \mathcal{S}_{n_{1}, \ldots, n_{d}} \rightarrow \mathbb{R}^{n_{1} \times \cdots \times n_{d}},\left(\mathcal{A}_{1}, \ldots, \mathcal{A}_{r}\right) \mapsto \mathcal{A}_{1}+\cdots+\mathcal{A}_{r}
$$

Then, under Assumption 1, there exists an open dense subset $\mathcal{N}_{r ; n_{1}, \ldots, n_{d}}$ of $\sigma_{r ; n_{1}, \ldots, n_{d}}$ such that for all $\mathcal{A} \in \mathcal{N}_{r ; n_{1}, \ldots, n_{d}}$ we have $\left|\Phi^{-1}(\mathcal{A})\right|=r$ ! by Beltrán et al. [11, Proposition 4.5-4.7]. ${ }^{2}$ In particular, the points in the fiber are isolated, so there is a local inverse map $\Phi_{a}^{-1}$ of $\Phi$ for each $a \in \Phi^{-1}(\mathcal{A})$. Recall from [20] that the condition number of the CPD at $\mathcal{A} \in \mathcal{N}_{r ; n_{1}, \ldots, n_{d}}$ is then the condition number (in the classic sense of Rice [61]; see also [28,69]) of any of these local inverses:

\footnotetext{
2 The preimage of an $r$-identifiable tensor under the map $\Phi$ consists of the $r$ ! permutations of the summands.

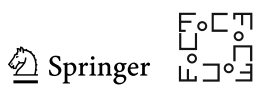




$$
\begin{aligned}
\kappa(\mathcal{A}) & :=\lim _{\epsilon \rightarrow 0} \sup _{\|\Delta \mathcal{A}\|_{<\epsilon \text { s.t. }} \mathcal{A}+\Delta \mathcal{A} \in \sigma_{r ; n_{1}, \ldots, n_{d}}} \frac{\left\|\Phi_{a}^{-1}(\mathcal{A})-\Phi_{a}^{-1}(\mathcal{A}+\Delta \mathcal{A})\right\|}{\|\Delta \mathcal{A}\|} \\
& =\left\|\mathrm{d}_{\mathcal{A}} \Phi_{a}^{-1}\right\|_{2},
\end{aligned}
$$

where $a \in \Phi^{-1}(\mathcal{A})$ is arbitrary; it is a corollary of Breiding and Vannieuwenhoven [20, Theorem 1.1] that the above definition does not depend on the choice of $a$. Herein, $\|\cdot\|$ in the denominator is the Euclidean norm induced by the ambient $\mathbb{R}^{n_{1} \times \cdots \times n_{d}}$, and the norm in the numerator is the product norm of the Euclidean norms inherited from the ambient $\mathbb{R}^{n_{1} \times \cdots \times n_{d}}$ 's. The right-hand side $\left\|\mathrm{d}_{\mathcal{A}} \Phi_{a}^{-1}\right\|_{2}$ is the spectral norm of the derivative of $\Phi_{a}^{-1}$ at $\mathcal{A}$. See Sect. 2 for more details. By Breiding and Vannieuwenhoven [20, Proposition 4.4], the condition number $\kappa(\mathcal{A})$ does not depend on the norm of $\mathcal{A}$ : $\kappa(t \mathcal{A})=\kappa(\mathcal{A})$ for $t \in \mathbb{R} \backslash\{0\}$.

Remark 1 We did not specify the value of the condition number for $\mathcal{A} \in \sigma_{r ; n_{1}, \ldots, n_{d}} \backslash \mathcal{N}_{r ; n_{1}, \ldots, n_{d}}$. The main reason is that our analysis is independent of the values that the condition number takes on this set of measure zero, so that for simplicity we decided against including the more complicated general case where there can be several distinct elements in the preimage.

\subsection{Main Results}

The goal of this paper is to study the average condition number relative to "reasonable" density functions. By this, we mean probability distributions $\hat{\rho}$ that are comparable to the standard Gaussian density $\rho$ : There exist positive constants $c_{1}, c_{2}$ such that $c_{1} \leq \frac{\hat{\rho}}{\rho} \leq c_{2}$. The main result, Theorem 4, applies, among others, for all distributions $\hat{\rho}$ comparable to the following Gaussian density defined on the set of bounded rank tensors $\sigma_{r ; n_{1}, \ldots, n_{d}}$.

Definition 1 (Gaussian identifiable tensors) We define a random variable $\mathcal{A}$ on $\sigma_{r ; n_{1}, \ldots, n_{d}}$ by specifying its density as

$$
\rho(\mathcal{A}):=\left(C_{r ; n_{1}, \ldots, n_{d}}\right)^{-1} e^{-\frac{\|\mathfrak{A}\|^{2}}{2}}, \quad \text { where } C_{r ; n_{1}, \ldots, n_{d}}=\int_{\sigma_{r ; n_{1}, \ldots, n_{d}}} e^{-\frac{\|\mathfrak{A}\|^{2}}{2}} \mathrm{~d} \mathcal{A}
$$

is the normalization constant. Under Assumption 1 , if $\mathcal{A} \in \sigma_{r ; n_{1}, \ldots, n_{d}}$ and $\mathcal{A} \sim \rho$, we say that $\mathcal{A}$ is a Gaussian Identifiable Tensor (GIT) of rank $r$.

Remark 2 Suppose that $r$ is a typical rank of tensors in $\mathbb{R}^{n_{1} \times \cdots \times n_{d}}$. This means that $\sigma_{r ; n_{1}, \ldots, n_{d}}$ contains a Euclidean open subset of $\mathbb{R}^{n_{1} \times \cdots \times n_{d}}$ and is of maximum dimension $n_{1} \cdots n_{d}$. Then, the distribution defined in Definition 1 is a conditional probability distribution: A GIT $\mathcal{A}$ of rank $r$ has the distribution $\mathcal{A} \sim(\mathcal{B} \mid \operatorname{rank}(\mathcal{B})=r)$, where $\mathcal{B}$ is a tensor with independent and identically distributed (i.i.d.) standard Gaussian entries. We exploit this fact in our numerical experiments to sample GITs using an acceptance-rejection method. 
We first state our results for the foregoing Gaussian density. At the end of this subsection, in Theorem 4, we generalize these results to other densities, including all densities comparable to the Gaussian density. Our first contribution is the following result. We prove it in Sect. 3.

Theorem 1 Let $\mathcal{A} \in \sigma_{2 ; n_{1}, \ldots, n_{d}}$ be a GIT of rank $r=2$. Then, $\mathbb{E} \kappa(\mathcal{A})=\infty$.

It should be mentioned that in our analysis we consider a small subset of $\sigma_{2 ; n_{1}, \ldots, n_{d}}$ and show that on this subset the condition number integrates to infinity. In particular, a weak average-case analysis as proposed in [3] would be of interest in this problem.

Under one additional assumption, we can extend the result from Theorem 1 to higher ranks. We prove the following theorem in Sect. 4.

Theorem 2 Let $n_{1}, \ldots, n_{d} \geq 3$. On top of Assumption 1 , we assume that $\sigma_{r-2, n_{1}-2, \ldots, n_{r}-2}$ is generically complex identifiable. Then, for a GIT $\mathcal{A} \in \sigma_{r ; n_{1}, \ldots, n_{d}}, r \geq 3$, we have $\mathbb{E} \kappa(\mathcal{A})=\infty$.

By Bocci et al. [17, Theorem 7.2], the assumptions of Theorem 2 are satisfied in a large number of cases. In fact, as the size of the tensor increases, the assumptions become weaker: When $n_{1} \geq n_{2} \geq \cdots \geq n_{d} \geq 2$, the conditions in Theorem 2 are satisfied for $r \leq \min \left(s_{1}, s_{2}\right)$ with

$$
\begin{aligned}
& s_{1}=\frac{n_{1} n_{2}-\left(n_{1}+n_{2}+n_{3}-2\right)}{n_{1} n_{2}} r_{n_{1}, \ldots, n_{d}}^{\mathrm{crit}} \\
& s_{2}=\frac{\left(n_{1}-2\right)\left(n_{2}-2\right)-\left(n_{1}+n_{2}+n_{3}-8\right)}{\left(n_{1}-2\right)\left(n_{2}-2\right)} r_{n_{1}-2, \ldots, n_{d}-2}^{\mathrm{crit}}+2 .
\end{aligned}
$$

Note that for large $n_{i}$, the second piece $s_{2}$ is the most restrictive. From Eq. (3), it is implied that $r_{n_{1}-2, \ldots, n_{d}-2}^{\text {crit }}=\left(1-\delta_{n_{1}, \ldots, n_{d}}\right) r_{n_{1}, \ldots, n_{d}}^{\text {crit }}$ with $\delta_{n_{1}, \ldots, n_{d}}=\mathcal{O}\left(\sum_{k=1}^{d} \frac{1}{n_{k}}\right)$. Therefore, we obtain the following asymptotically optimal result.

Corollary 1 Let $d \geq 3$ be fixed, and $n_{1} \geq n_{2} \geq \cdots \geq n_{d} \geq 2$. If $n_{1}, \ldots, n_{d} \rightarrow \infty$, then for a GIT $\mathcal{A} \in \sigma_{r ; n_{1}, \ldots, n_{d}}$ we have $\mathbb{E} \kappa(\mathcal{A})=\infty$ for all

$$
2 \leq r<\left(1-\epsilon_{n_{1}, \ldots, n_{d}}\right) r_{n_{1}, \ldots, n_{d}}^{c r i t},
$$

where $\lim _{n_{1}, \ldots, n_{d} \rightarrow \infty} \epsilon_{n_{1}, \ldots, n_{d}} \rightarrow 0$.

It follows from dimensionality arguments that if $r>r_{\text {crit }}$, then the addition map $\Phi$ does not have a local inverse. In fact, in this case all of the connected components in the fiber of $\Phi$ at $\mathcal{A} \in \sigma_{r ; n_{1}, \ldots, n_{d}}$ have positive dimension [46]. It follows from Breiding and Vannieuwenhoven [20] that the condition number of the tensor rank decomposition problem at each expression Eq. (1) of length $r$ of such a tensor $\mathcal{A}$ is $\infty$. In this case, $\kappa(\mathcal{A})=\infty$, regardless of how the tensor decomposition problem is defined ${ }^{3}$ when $\mathcal{A}$

3 This is exactly the concern of Remark 1: What computational problem are we interested in solving when
a tensor has several distinct CPDs? Are we interested in the CPD with the best sensitivity? Or the worst?
Or the expected condition number of one randomly chosen CPD in the fiber? This depends on the context.
The results of this paper are valid regardless of the particular variation of the problem one is interested in.

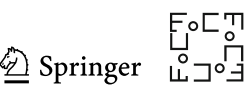


has multiple distinct decompositions; see also the discussion in [28, Remark 14.14]. In this case the average condition number is infinite, as well.

Our results lead us to the conjecture that the expected condition number is infinite, also without making the assumption from Theorem 2 and without any upper bound on the rank.

Conjecture 1 Let $\mathcal{A} \in \sigma_{r ; n_{1}, \ldots, n_{d}}$ be a GIT of rank $r \geq 2$. Then, $\mathbb{E} \kappa(\mathcal{A})=\infty$.

Corollary 1 proves this conjecture asymptotically, in practice leaving only a small range of ranks for which it might fail.

As mentioned above, it turns out that for GITs the expected angular condition number is not always infinite. Formally, the angular condition number is defined as follows: Let the canonical projection onto the sphere be $p: \mathbb{R}^{n_{1} \times \cdots \times n_{d}} \rightarrow \mathbb{S}\left(\mathbb{R}^{n_{1} \times \cdots \times n_{d}}\right)$. Then, the angular condition number of $\mathcal{A} \in \mathcal{N}_{r ; n_{1}, \ldots, n_{d}}$ is

$$
\kappa_{\text {ang }}(\mathcal{A}):=\lim _{\epsilon \rightarrow 0} \sup _{\substack{\|\Delta \mathcal{A}\|<\epsilon, \mathcal{A}+\Delta \mathcal{A} \in \sigma_{r ; n_{1}, \ldots, n_{d}}}} \frac{\left\|\left(p^{\times r} \circ \Phi_{a}^{-1}\right)(\mathcal{A})-\left(p^{\times r} \circ \Phi_{a}^{-1}\right)(\mathcal{A}+\Delta \mathcal{A})\right\|}{\|\Delta \mathcal{A}\|}
$$

where $\Phi_{a}^{-1}$ is an arbitrary local inverse of $\Phi$ with $\mathcal{A}=\Phi(a)$. As before we do not specify what happens on the measure-zero set $\sigma_{r ; n_{1}, \ldots, n_{d}} \backslash \mathcal{N}_{r ; n_{1}, \ldots, n_{d}}$, because it is not relevant for this paper. The angular condition number only accounts for the angular part of the CPD, i.e., the directions of the tensors, not for their magnitude, hence the name.

To distinguish the condition numbers Eqs. (5) and (6), we will refer to the condition number from Eq. 5 as the regular condition number. Oftentimes we even drop the clarification "regular."

Here is the result for $\kappa_{\text {ang }}(\mathcal{A})$ for tensors of rank two that we prove in Sect. 5.

Theorem 3 Let $\mathcal{A} \in \sigma_{2 ; n_{1}, \ldots, n_{d}}$ be a GIT of rank 2 . Then, $\mathbb{E} \kappa_{\mathrm{ang}}(\mathcal{A})<\infty$.

Unfortunately, we do not know if this theorem can be extended to higher rank tensors. However, based on our experiments in Sect. 7, we pose the following:

Conjecture 2 Let $\mathcal{A} \in \sigma_{r ; n_{1}, \ldots, n_{d}}$ be a GIT of rank $r$. Then, $\mathbb{E} \kappa_{\text {ang }}(\mathcal{A})<\infty$.

We finally observe that the foregoing main results are not limited to GITs. They are valid for a wide range of distributions of random tensors.

Theorem 4 Theorems 1, 2, Corollary 1 and Theorem 3 are still true if instead of GITs we take random tensors defined by a wide range of other probability distributions, including some of interest such as:

1. All probability distributions that are comparable to the standard Gaussian density $\rho$. This means that the random tensor $\mathcal{A}$ has a density $\hat{\rho}$ for which there exists positive constants $c_{1}, c_{2}$ such that $c_{1} \leq \frac{\hat{\rho}}{\rho} \leq c_{2}$.

2. Uniformly randomly chosen $\mathcal{A}$ in the unit sphere $\mathbb{S}\left(\sigma_{r}\right)$.

3. Uniformly randomly chosen $\mathcal{A}$ in the unit ball $\left\{\mathcal{A} \in \sigma_{r}:\|\mathcal{A}\| \leq 1\right\}$. 


\subsection{Organization of the Article}

The rest of the article is organized as follows. In the next section, we give some preliminary material. Thereafter, in Sects. 3-6, we successively prove Theorems 1-4. In Sect. 7, we present numerical experiments supporting our main results. Finally, in Appendices A-C we give proofs for several lemmata that we need in the other sections.

\section{Notation and Preliminaries}

\subsection{Notation}

We will use the following typographic conventions for convenience: Vectors are typeset in a bold face $(\mathbf{a}, \mathbf{b})$, matrices in upper case $(A, B)$, tensors in a calligraphic font $(\mathcal{A}$, $\mathcal{B})$, and manifolds and linear spaces in a different calligraphic font $(\mathcal{A}, \mathcal{B})$.

The positive integer $d \geq 2$ is reserved for the order of a tensor, $n_{1}, \ldots, n_{d} \geq 2$ are its dimensions, and $r \geq 1$ is its rank. The following integers are used throughout the paper:

$$
\Sigma:=1+\sum_{k=1}^{d}\left(n_{k}-1\right) \text { and } \Pi:=\prod_{k=1}^{d} n_{k}
$$

they correspond to the dimension of the Segre manifold $\mathcal{S}_{n_{1}, \ldots, n_{d}}$ and the dimension of the ambient space $\mathbb{R}^{n_{1} \times \cdots \times n_{d}}$, respectively. The symmetric group on $r$ elements is denoted by $\mathfrak{S}_{r}$.

We work exclusively with real vector spaces, for which $\langle\cdot, \cdot\rangle$ denotes the Euclidean inner product and $\|\cdot\|$ always denotes the associated norm. We will switch freely between the finite-dimensional vector spaces $\mathbb{R}^{n_{1} \cdots n_{d}}$ and $\mathbb{R}^{n_{1} \times \cdots \times n_{d}}$ for representing tensors in the abstract vector space $\mathbb{R}^{n_{1}} \otimes \cdots \otimes \mathbb{R}^{n_{d}}$. By the above choice of norms, all of these finite-dimensional Hilbert spaces are isometric; specifically, if $\mathcal{A} \in \mathbb{R}^{n_{1}} \otimes$ $\cdots \otimes \mathbb{R}^{n_{d}}$ and $\mathbf{a} \in \mathbb{R}^{n_{1} \cdots n_{d}}$ is its coordinate array with respect to an orthogonal basis, then $\|\mathcal{A}\|=\|\mathbf{a}\|$. Similarly, if the coordinates a are reshaped into a multidimensional array $A \in \mathbb{R}^{n_{1} \times \cdots \times n_{d}}$, then $\|A\|=\|\mathcal{A}\|=\|\mathbf{a}\|$. It is important to note that this notation can conflict with the usual meaning of $\|A\|$ when $d=2$; to distinguish the spectral norm from the standard norm in this paper, we write $\|A\|_{2}$ for the former; see Eq. (7).

For matrices $U_{1} \in \mathbb{R}^{m_{1} \times n_{1}}, \ldots, U_{d} \in \mathbb{R}^{m_{d} \times n_{d}}$, the tensor product $U_{1} \otimes \cdots \otimes U_{d}$ acts on rank-1 tensors as follows:

$$
\left(U_{1} \otimes \cdots \otimes U_{d}\right)\left(\mathbf{u}^{1} \otimes \cdots \otimes \mathbf{u}^{d}\right)=\left(U_{1} \mathbf{u}^{1}\right) \otimes \cdots \otimes\left(U_{d} \mathbf{u}^{d}\right)
$$

By the universal property [44], this extends to a linear map $\mathbb{R}^{n_{1}} \otimes \cdots \otimes \mathbb{R}^{n_{d}} \rightarrow$ $\mathbb{R}^{m_{1}} \otimes \cdots \otimes \mathbb{R}^{m_{d}}$. Note that we can view $U_{1} \otimes \cdots \otimes U_{d}$ as a matrix in $\mathbb{R}^{\left(m_{1} \cdots m_{d}\right) \times\left(n_{1} \cdots n_{d}\right)}$.

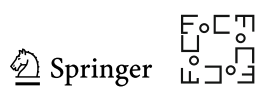


For any subset $U \subset V$ of a normed vector space $V$, we define the sphere over $U$ as

$$
\mathbb{S}(U):=\left\{\frac{\mathbf{u}}{\|\mathbf{u}\|} \mid \mathbf{u} \in U \backslash\{0\}\right\} \subset V .
$$

In particular, the unit sphere in $\mathbb{R}^{n}$ is denoted by $\mathbb{S}\left(\mathbb{R}^{n}\right)$.

Given an $m \times n$ matrix $R$ or a linear operator $R: \mathbb{R}^{n} \rightarrow \mathbb{R}^{m}$, we denote the pseudo-inverse by $R^{\dagger}$. The spectral norm and smallest singular value of $R$ are denoted respectively by

$$
\|R\|_{2}:=\max _{\mathbf{v} \in \mathbb{R}^{n}} \frac{\|R \mathbf{v}\|}{\|\mathbf{v}\|} \quad \text { and } \quad \zeta_{\min }(R):=\min _{\mathbf{v} \in \mathbb{R}^{n}} \frac{\|R \mathbf{v}\|}{\|\mathbf{v}\|} .
$$

A special role will be played in this paper by the product of all but the smallest singular values of $R$, which we denote by $q(R)$. In other words, if $R$ is injective, then

$$
q(R):=\varsigma_{1}(R) \cdots \varsigma_{n-1}(R)=\frac{\sqrt{\operatorname{det}\left(R^{T} R\right)}}{\varsigma_{\min }(R)},
$$

where $R^{T}$ is the transposed matrix (operator) and $\zeta_{i}(R)$ is the $i$ th largest singular value of $R$.

\subsection{Differential Geometry}

In this article, we only consider submanifolds of Euclidean spaces; see, e.g., [58] for the general definitions. A smooth $\left(C^{\infty}\right)$ manifold is a topological manifold with a smooth structure, in the sense of Lee [58]. The tangent space $\mathrm{T}_{x} \mathcal{M}$ at $x$ to an embedded $n$-dimensional smooth submanifold $\mathcal{M} \subset \mathbb{R}^{N}$ is the set

$$
\left\{\mathbf{v} \in \mathbb{R}^{N} \mid \exists \text { a smooth curve } \gamma(t) \subset \mathcal{M} \text { with } \gamma(0)=x: \mathbf{v}=\left.\frac{\mathrm{d}}{\mathrm{d} t}\right|_{t=0} \gamma(t)\right\} .
$$

At every point $x \in \mathcal{M}$, there exist open neighborhoods $\mathcal{V} \subset \mathcal{M}$ and $\mathcal{U} \subset \mathrm{T}_{x} \mathcal{M}$ of $x$, and a bijective smooth map $\phi: \mathcal{V} \rightarrow \mathcal{U}$ with smooth inverse. The tuple $(\mathcal{V}, \phi)$ is a coordinate chart of $\mathcal{M}$. A smooth map between manifolds $F: \mathcal{M} \rightarrow \mathcal{N}$ is a map such that for every $x \in \mathcal{M}$ and coordinate chart $(\mathcal{V}, \phi)$ containing $x$, and every coordinate chart $(\mathcal{W}, \psi)$ containing $F(x)$, we have that $\psi \circ F \circ \phi^{-1}: \phi(\mathcal{U}) \rightarrow \psi(F(\mathcal{U}))$ is a smooth map. The derivative of $F$ can be defined as the linear map $\mathrm{d}_{x} F: \mathrm{T}_{x} \mathcal{M} \rightarrow$ $\mathrm{T}_{F(x)} \mathcal{N}$ taking the tangent vector $\mathbf{v} \in \mathrm{T}_{x} \mathcal{M}$ to $\left.\frac{\mathrm{d}}{\mathrm{d} t}\right|_{t=0} F(\gamma(t)) \in \mathrm{T}_{F(x)} \mathcal{N}$ where $\gamma(t) \subset \mathcal{M}$ is a curve with $\gamma(0)=x$ and $\gamma^{\prime}(0)=\mathbf{v}$. If $\operatorname{dim} \mathcal{M}=\operatorname{dim} \mathcal{N}$ and if $\mathrm{d}_{x} F$ has full rank, there is a neighborhood $\mathcal{W} \subset \mathcal{M}$ on which $F$ is invertible and its inverse is also smooth; that is, $F$ is a diffeomorphism between $\mathcal{W}$ and $F(\mathcal{W})$. If this property holds for all $x \in \mathcal{M}$, then $F$ is called a local diffeomorphism.

A differentiable submanifold $\mathcal{M} \subset \mathbb{R}^{N}$ can be equipped with a Riemannian metric $g$, turning it into a Riemannian manifold, allowing for the computation of integrals. 
The manifolds in this paper are all embedded submanifolds of Euclidean space, so the Riemannian metric for us will always be the metric inherited from the ambient space.

\subsection{The Manifold of $r$-Nice Tensors}

As in the introduction, the Segre manifold is

$$
\mathcal{S}_{n_{1}, \ldots, n_{d}}=\left\{\mathbf{u}^{1} \otimes \cdots \otimes \mathbf{u}^{d} \mid \mathbf{u}^{k} \in \mathbb{R}^{n_{k}} \backslash\{0\}\right\}
$$

It is a smooth manifold of dimension $\Sigma$. Its tangent space is given by

$$
\mathrm{T}_{\mathbf{u}^{1} \otimes \cdots \otimes \mathbf{u}^{d}} \mathcal{S}_{n_{1}, \ldots, n_{d}}=\mathbb{R}^{n_{1}} \otimes \mathbf{u}^{2} \otimes \cdots \otimes \mathbf{u}^{d}+\cdots+\mathbf{u}^{1} \otimes \cdots \otimes \mathbf{u}^{d-1} \otimes \mathbb{R}^{n_{d}}
$$

note that this is not a direct sum.

The Euclidean inner product between rank-1 tensors is conveniently computed by the following formula (see, e.g., [45]):

$$
\left\langle\mathbf{u}^{1} \otimes \cdots \otimes \mathbf{u}^{d}, \mathbf{v}^{1} \otimes \cdots \otimes \mathbf{v}^{d}\right\rangle=\prod_{i=1}^{d}\left\langle\mathbf{u}^{i}, \mathbf{v}^{i}\right\rangle .
$$

The set of tensors of rank at most $r$ is denoted by

$$
\sigma_{r ; n_{1}, \ldots, n_{d}}=\left\{\mathcal{A} \in \mathbb{R}^{n_{1} \times \cdots \times n_{d}} \mid \operatorname{rank}(\mathcal{A}) \leq r\right\}
$$

it is a semialgebraic set of dimension at $\operatorname{most} \min \{r \Sigma, \Pi\}$; see, e.g., [60]. Under Assumption 1 , the dimension of $\sigma_{r ; n_{1}, \ldots, n_{d}}$ is exactly $r \Sigma$.

In [11, Section 4], we introduced an open dense subset of $\sigma_{r ; n_{1}, \ldots, n_{d}}$ with favorable differential-geometric properties. We called it the manifold of $r$-nice tensors in [11, Definition 4.2]. Below, we present a slightly modified definition that is suitable for our present purpose; it eliminates conditions (4) and (5) from Beltrán et al. [11, Definition 4.2].

In what follows, we denote the real closure in the Zariski topology of a subset $A \subset \mathbb{R}^{\Pi}$ by $\bar{A}$. This is the real algebraic variety $\bar{A}:=\bar{A}^{\mathbb{C}} \cap \mathbb{R}^{\Pi}$, where $\bar{A}^{\mathbb{C}}$ is the closure of $A$ in the Zariski topology in $\mathbb{C}^{\Pi}$. By Whitney [70, Lemma 8], the real dimension of $\bar{A}$ equals the complex dimension of $\bar{A}$.

Definition 2 Recall the addition map $\Phi$ defined in Eq. (4). Let $\mathcal{M}_{r ; n_{1}, \ldots, n_{d}} \subset$ $\left(\mathcal{S}_{n_{1}, \ldots, n_{d}}\right)^{\times r}$ be the subset of $r$-tuples $a:=\left(\mathcal{A}_{1}, \ldots, \mathcal{A}_{r}\right)$ of rank-1 tensors satisfying all of the following properties:

1. $\Phi(a)$ is a smooth point of the algebraic variety $\overline{\sigma_{r ; n_{1}, \ldots, n_{d}}}$;

2. $\Phi(a)$ is complex $r$-identifiable; and

3. $\kappa(\Phi(a))<\infty$.

The set of $r$-nice tensors is $\mathcal{N}_{r ; n_{1}, \ldots, n_{d}}:=\Phi\left(\mathcal{M}_{r ; n_{1}, \ldots, n_{d}}\right)$.

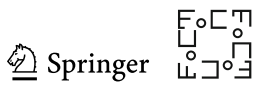


Remark that the third item in the definition is well defined because of the second item.

Proposition 1 If Assumption 1 holds, then the following statements are true:

1. $\mathcal{M}_{r ; n_{1}, \ldots, n_{d}}$ and $\mathcal{N}_{r ; n_{1}, \ldots, n_{d}}$ are smooth manifolds of dimension $r \Sigma$;

2. $\mathcal{M}_{r ; n_{1}, \ldots, n_{d}}$ is Zariski-open in $\left(\mathcal{S}_{n_{1}, \ldots, n_{d}}\right)^{\times r}$;

3. $\mathcal{N}_{r ; n_{1}, \ldots, n_{d}}$ is Zariski-open in $\sigma_{r ; n_{1}, \ldots, n_{d}}$;

4. the addition map $\left.\Phi\right|_{\mathcal{M}_{r ; n_{1}, \ldots, n_{d}}}$ is a global diffeomorphism onto its image;

5. $\mathcal{N}_{r ; n_{1}, \ldots, n_{d}}$ is closed under multiplication by nonzero scalars; and

6. $\mathcal{M}_{r ; n_{1}, \ldots, n_{d}} \subset \mathbb{R}^{n_{1} \times \cdots \times n_{d}} \times \cdots \times \mathbb{R}^{n_{1} \times \cdots \times n_{d}}$ and $\mathcal{N}_{r ; n_{1}, \ldots, n_{d}} \subset \mathbb{R}^{n_{1} \times \cdots \times n_{d}}$ are embedded submanifolds.

Proof Items 1, 2, 3, and 6 are proved as follows. Let $X_{1}$ and $X_{2}$ be, respectively, the set of tensors in $\sigma_{r ; n_{1}, \ldots, n_{d}}$ which are not complex $r$-identifiable and which are not smooth points of $\overline{\sigma_{r ; n_{1}, \ldots, n_{d}}}$. Both are Zariski-closed in $\overline{\sigma_{r ; n_{1}, \ldots, n_{d}}}$ under Assumption 1, and hence so are the preimages $\Phi^{-1}\left(X_{1}\right)$ and $\Phi^{-1}\left(X_{2}\right)$. Moreover, the third defining condition of $\mathcal{M}_{r ; n_{1}, \ldots, n_{d}}$ is also Zariski-closed in $\left(\mathcal{S}_{n_{1}, \ldots, n_{d}}\right)^{\times r}$ from the explicit formula for the condition number Eq. (12). Hence, $\mathcal{M}_{r ; n_{1}, \ldots, n_{d}}$ is Zariski-open. An open subset of an embedded submanifold is itself an embedded submanifold so the claim for $\mathcal{M}_{r ; n_{1}, \ldots, n_{d}}$ is proved. Moreover, the dimension of the complement of $\mathcal{M}_{r ; n_{1}, \ldots, n_{d}}$ is at most $r \Sigma-1$ and so its image by the rational map $\Phi$ is contained in an algebraic set of dimension at most $r \Sigma-1$, thus proving that $\mathcal{N}_{r ; n_{1}, \ldots, n_{d}}$ is also Zariski-open and indeed an embedded submanifold of the set of smooth points of $\overline{\sigma_{r ; n_{1}, \ldots, n_{d}}}$, which is itself an embedded submanifold of its affine ambient space, see [12, Proposition 3.2.9].

The fourth item is due to the definition of the condition number, the fact that it is finite on $\mathcal{N}_{r ; n_{1}, \ldots, n_{d}}$ by Definition 2, and the injectivity of $\left.\Phi\right|_{\mathcal{M}_{r ; n_{1}, \ldots, n_{d}}}$ by Definition $2(2)$.

The fifth item follows by noting that the three defining properties of $\mathcal{N}_{r ; n_{1}, \ldots, n_{d}}$ are all true independent of a nonzero scaling.

Remark 3 The definition of $r$-nice tensors in [11, Definition 4.2] involves two more requirements, but those are not needed here.

Since the tangent space of $\mathcal{N}_{r ; n_{1}, \ldots, n_{d}}$ at a point is the image of the derivative of the local diffeomorphism $\Phi$, we have the following characterization:

$$
\mathrm{T}_{\mathcal{A}} \mathcal{N}_{r, n_{1}, \ldots, n_{d}}=\mathrm{T}_{\mathcal{A}_{1}} \mathcal{S}_{n_{1}, \ldots, n_{d}}+\cdots+\mathrm{T}_{\mathcal{A}_{r}} \mathcal{S}_{n_{1}, \ldots, n_{d}}, \text { for } \mathcal{A}=\mathcal{A}_{1}+\cdots+\mathcal{A}_{r}
$$

\subsection{Sensitivity of CPDs}

The condition number of the problem of computing the rank-1 terms of a CPD of a tensor was studied in a general setting in [20]; the following characterization of the condition number is Theorem 1.1 of [20]. Let $\mathcal{A}=\mathcal{A}_{1}+\cdots+\mathcal{A}_{r} \in \mathcal{N}_{r, n_{1}, \ldots, n_{d}}$, where the $\mathcal{A}_{i} \in \mathcal{S}_{n_{1}, \ldots, n_{d}}$ are rank-1 tensors. For each $i$ let $U_{i}$ be a matrix whose columns form an orthonormal basis of $\mathrm{T}_{\mathscr{A}_{i}} \mathcal{S}_{n_{1}, \ldots, n_{d}}$. Then,

$$
\kappa(\mathcal{A})=\frac{1}{\varsigma_{\min }\left(\left[U_{1}, \ldots, U_{r}\right]\right)} .
$$


The matrix $U=\left[U_{1}, \ldots, U_{r}\right] \in \mathbb{R}^{\Pi \times r \Sigma}$ is also called a Terracini matrix. An explicit expression for the $U_{i}$ 's is given in [20, equation (5.1)].

Since $\mathcal{A}$ uniquely depends on $a:=\left(\mathcal{A}_{1}, \ldots, \mathcal{A}_{r}\right) \in \mathcal{S}_{n_{1}, \ldots, n_{d}}^{\times r}$, we can view the condition number of $\mathcal{A} \in \mathcal{N}_{r, n_{1}, \ldots, n_{d}}$ as a function of $a$ :

$$
\kappa(a):=\frac{1}{\varsigma_{\min }\left(\left[U_{1}, \ldots, U_{r}\right]\right)},
$$

where the matrices $U_{i}$ are as before. The benefit of Eq. (13) is that it is well-defined for any tuple $a \in \mathcal{S}_{n_{1}, \ldots, n_{d}}^{\times r}$ (and not just those mapping into $\mathcal{N}_{r, n_{1}, \ldots, n_{d}}$ ).

\subsection{Integrals}

For fixed $t \in(0,1]$ and a point $\mathbf{y} \in \mathbb{S}\left(\mathbb{R}^{n}\right)$, the spherical cap of radius $t$ around $\mathbf{y}$ is defined as $\operatorname{cap}(\mathbf{y}, t):=\left\{\mathbf{x} \in \mathbb{S}\left(\mathbb{R}^{n}\right):\langle\mathbf{x}, \mathbf{y}\rangle>\sqrt{1-t^{2}}\right\}$. Its volume satisfies

$$
c_{1}(n) t^{n-1} \leq \operatorname{vol}(\operatorname{cap}(\mathbf{y}, t)) \leq c_{2}(n) t^{n-1}
$$

for some positive constants $0<c_{1}(n)<c_{2}(n)$.

The following general lemma will be useful later.

Lemma 2 Let $u, v>0$ be fixed. Then, $0<\int_{0}^{\infty} t^{u} e^{-\frac{(t+v)^{2}}{2}} \mathrm{~d} t<\infty$.

Proof It is clear that the integral is not zero. Furthermore, since $(t+v)^{2}>t^{2}+v^{2}$ for $t, v>0$, we see that $\int_{0}^{\infty} t^{u} e^{-\frac{(t+v)^{2}}{2}} \mathrm{~d} t \leq \int_{0}^{\infty} t^{u} e^{-\frac{t^{2}+v^{2}}{2}} \mathrm{~d} t=e^{-\frac{v^{2}}{2}} \sqrt{2}{ }^{u-1} \Gamma\left(\frac{u+1}{2}\right)$, which is finite.

\subsection{The Coarea Formula}

Let $\mathcal{M}$ and $\mathcal{N}$ be submanifolds of $\mathbb{R}^{n}$ of equal dimension, and let $F: \mathcal{M} \rightarrow \mathcal{N}$ be a smooth surjective map. A point $y \in \mathcal{N}$ is called a regular value of $F$ if for all points $x \in F^{-1}(y)$ the differential $\mathrm{d}_{x} F$ is of full rank. The preimage $F^{-1}(y)$ of a regular value $y$ is a discrete set of points. Let $\left|F^{-1}(y)\right|$ be the number of elements in this preimage. Then, the coarea formula [52] states that for every integrable function $g$ we have

$$
\int_{\mathcal{N}}\left|F^{-1}(y)\right| g(y) \mathrm{d} y=\int_{\mathcal{M}} \operatorname{Jac}(F)(x) g(F(x)) \mathrm{d} x,
$$

where $\operatorname{Jac}(F)(x):=\left|\operatorname{det} \mathrm{d}_{x} F\right|$ is the Jacobian determinant of $F$ at $x$. Note that almost all $y \in \mathcal{N}$ are regular values of $F$ by Sard's theorem [58, Theorem 6.10]. Hence, integrating over $\mathcal{N}$ is the same as integrating over all regular values of $F$.

Remark 4 In [52], the coarea formula is given in the more general case when $\operatorname{dim} \mathcal{M} \geq$ $\operatorname{dim} \mathcal{N}$. In this article, we only need the case when the $\operatorname{dimension}$ of $\operatorname{dim} \mathcal{M}$ and $\operatorname{dim} \mathcal{N}$ coincide. Moreover, if $F$ is injective, then Eq. (15) reduces to the well-known changeof-variables formula.

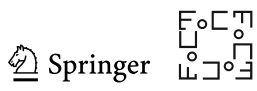




\section{The Average Condition Number of Gaussian Tensors of Rank Two}

The goal of this section is to prove Theorem 1. We will proceed in three steps. First, the 2-nice tensors are conveniently parameterized via elementary manifolds such as one-dimensional intervals and spheres in Sect. 3.1. Second, the Jacobian determinant of this map is computed in Sect. 3.2. Third, the integral can be bounded from below with the help of a few technical auxiliary lemmas in Sect. 3.3. In the next section, we will exploit Theorem 1 for generalizing the argument to most higher ranks. To simplify notation, in this section we let

$$
\mathcal{S}:=\mathcal{S}_{n_{1}, \ldots, n_{d}}, \sigma_{2}:=\sigma_{2, n_{1}, \ldots, n_{d}}, \mathcal{N}_{2}:=\mathcal{N}_{2, n_{1}, \ldots, n_{d}} \text { and } \mathcal{M}_{2}:=\mathcal{M}_{2, n_{1}, \ldots, n_{d}}
$$

\subsection{Parameterizing 2-Nice Tensors}

Let

$$
\mathcal{P}:=\mathbb{S}\left(\mathbb{R}^{n_{1}}\right) \times \cdots \times \mathbb{S}\left(\mathbb{R}^{n_{d}}\right)
$$

and consider the next parameterization of the Segre manifold:

$$
\psi:(0, \infty) \times \mathcal{P} \rightarrow \mathcal{S},\left(\lambda, \mathbf{u}^{1}, \ldots, \mathbf{u}^{d}\right) \mapsto \lambda \cdot \mathbf{u}^{1} \otimes \cdots \otimes \mathbf{u}^{d}
$$

The preimage of $\mathcal{A} \in \mathcal{S}$ has cardinality $\left|\psi^{-1}(\mathcal{A})\right|=2^{d-1}$. By composing $\Psi:=\psi \times \psi$ with the addition map from Eq. (4), we get the following alternative representation of tensors of rank bounded by 2 :

$(\Phi \circ \Psi)\left(\left(\lambda, \mathbf{u}^{1}, \ldots, \mathbf{u}^{d}\right),\left(\mu, \mathbf{v}^{1}, \ldots, \mathbf{v}^{d}\right)\right)=\lambda \cdot \mathbf{u}^{1} \otimes \cdots \otimes \mathbf{u}^{d}+\mu \cdot \mathbf{v}^{1} \otimes \cdots \otimes \mathbf{v}^{d}$

We would like to apply the coarea formula Eq. (15) to pull back the integral of $\kappa(\mathcal{A}) e^{-\frac{\|\mathfrak{A}\|}{2}}$ over $\sigma_{2}$ via the parameterization $\Phi \circ \Psi$. However, $\sigma_{2}$ in general is not a manifold, so the formula does not apply. Nevertheless, we can use the manifold $\mathcal{N}_{2}$ of 2-nice tensors instead. By Proposition $1(3), \mathcal{N}_{2}$ is Zariski open in $\sigma_{2}$, so that

$$
\mathbb{E} \kappa(\mathcal{A})=\frac{1}{C_{2}} \int_{\sigma_{2}} \kappa(\mathcal{A}) e^{-\frac{\|\mathfrak{H}\|^{2}}{2}} \mathrm{~d} \mathcal{A}=\frac{1}{C_{2}} \int_{\mathcal{N}_{2}} \kappa(\mathcal{A}) e^{-\frac{\|\mathfrak{A}\|^{2}}{2}} \mathrm{~d} \mathcal{A},
$$

where $C_{2}:=C_{2 ; n_{1}, \ldots, n_{d}}$ is as in Definition 1. By applying the coarea formula Eq. (15) to the smooth map $\left.\Phi\right|_{\mathcal{M}_{2}}$, we get

$$
\begin{aligned}
\int_{\mathcal{N}_{2}} \kappa(\mathcal{A}) e^{-\frac{\|\mathscr{A}\|^{2}}{2}} \mathrm{~d} \mathcal{A} & =\frac{1}{2} \int_{\mathcal{N}_{2}}\left|\Phi^{-1}(\mathcal{A})\right| \kappa(\mathcal{A}) e^{-\frac{\|\mathscr{A}\|^{2}}{2}} \mathrm{~d} \mathcal{A} \\
& =\frac{1}{2} \int_{\mathcal{M}_{2}} \operatorname{Jac}(\Phi)\left(\mathcal{A}_{1}, \mathcal{A}_{2}\right) \kappa\left(\mathcal{A}_{1}+\mathcal{A}_{2}\right) e^{-\frac{\left\|\mathcal{A}_{1}+\mathcal{A}_{2}\right\|^{2}}{2}} \mathrm{~d} \mathcal{A}_{1} \mathrm{~d} \mathcal{A}_{2}
\end{aligned}
$$


where $\operatorname{Jac}(\Phi)\left(\mathcal{A}_{1}, \mathcal{A}_{1}\right)$ is the Jacobian determinant of $\Phi$ at $\left(\mathcal{A}_{1}, \mathcal{A}_{1}\right)$. In the first equality, we used $\left|\Phi^{-1}(\mathcal{A})\right|=2$ for 2 -identifiable tensors; indeed, we have that $\Phi\left(\mathcal{A}_{1}, \mathcal{A}_{2}\right)=$ $\Phi\left(\mathcal{A}_{2}, \mathcal{A}_{1}\right)=\mathcal{A}$ and $\mathcal{A}_{1} \neq \mathcal{A}_{2}$ because $\mathcal{A} \in \mathcal{N}_{2}$ has rank equal to 2 .

In the following, we switch to the notation from Eq. (13): $\kappa\left(\mathcal{A}_{1}+\mathcal{A}_{2}\right)=\kappa\left(\mathcal{A}_{1}, \mathcal{A}_{2}\right)$. Since $\mathcal{M}_{2}$ is also Zariski open in $\mathcal{S} \times \mathcal{S}$ by Proposition 1(2), we may replace the integral over $\mathcal{M}_{2}$ by an integral over $\mathcal{S} \times \mathcal{S}$, thus obtaining

$$
\mathbb{E} \kappa(\mathcal{A})=\frac{1}{2 C_{2}} \int_{\mathcal{S} \times \mathcal{S}} \operatorname{Jac}(\Phi)\left(\mathcal{A}_{1}, \mathcal{A}_{1}\right) \kappa\left(\mathcal{A}_{1}, \mathcal{A}_{2}\right) e^{-\frac{\left\|\mathcal{A}_{1}+\mathcal{H}_{2}\right\|^{2}}{2}} \mathrm{~d} \mathcal{A}_{1} \mathrm{~d} \mathcal{A}_{2}
$$

We use the coarea formula again, but this time for $\Psi=\psi \times \psi$, where $\psi$ is the parameterization from Eq. (17). Note that for $\left(\mathcal{A}_{1}, \mathcal{A}_{2}\right) \in \mathcal{M}_{2}$ we have $\left|\Psi^{-1}\left(\mathcal{A}_{1}, \mathcal{A}_{2}\right)\right|=$ $2^{2 d-2}$. We get

$$
\begin{aligned}
\mathbb{E} \kappa(\mathcal{A}) & =\frac{1}{2 C_{2}} \int_{\mathcal{S} \times \mathcal{S}} \operatorname{Jac}(\Phi)\left(\mathcal{A}_{1}, \mathcal{A}_{2}\right) \kappa\left(\mathcal{A}_{1}, \mathcal{A}_{2}\right) e^{-\frac{\left\|\mathcal{A}_{1}+\mathcal{A}_{2}\right\|^{2}}{2}} \mathrm{~d} \mathcal{A}_{1} \mathrm{~d} \mathcal{A}_{2} \\
& =\frac{1}{2^{2 d-1} C_{2}} \int_{\mathcal{S} \times \mathcal{S}}\left|\Psi^{-1}\left(\mathcal{A}_{1}, \mathcal{A}_{2}\right)\right| \operatorname{Jac}(\Phi)\left(\mathcal{A}_{1}, \mathcal{A}_{2}\right) \kappa\left(\mathcal{A}_{1}, \mathcal{A}_{2}\right) e^{-\frac{\left\|\mathcal{A}_{1}+\mathcal{A}_{2}\right\|^{2}}{2}} \mathrm{~d} \mathcal{A}_{1} \mathrm{~d} \mathcal{A}_{2} \\
& =\frac{1}{2^{2 d-1} C_{2}} \int_{((0, \infty) \times \mathcal{P})^{\times 2}} \operatorname{Jac}(\Phi \circ \Psi)(a, b) \kappa(\psi(a), \psi(b)) e^{-\frac{\|\psi(a)+\psi(b)\|^{2}}{2}} \mathrm{~d} a \mathrm{~d} b
\end{aligned}
$$

where $a=\left(\lambda, \mathbf{u}^{1}, \ldots, \mathbf{u}^{d}\right)$ and $\sigma=\left(\mu, \mathbf{v}^{1}, \ldots, \mathbf{v}^{d}\right)$ are both tuples in $(0, \infty) \times \mathcal{P}$. Next, we compute the Jacobian determinant $\operatorname{Jac}(\Phi \circ \Psi)(a, b)$.

\subsection{Computing the Jacobian Determinant}

Note that the dimension of the domain of $\Phi \circ \Psi$ is equal to $2 \Sigma$. As above, let $a=$ $\left(\lambda, \mathbf{u}^{1}, \ldots, \mathbf{u}^{d}\right)$ and $\sigma=\left(\mu, \mathbf{v}^{1}, \ldots, \mathbf{v}^{d}\right)$ be tuples in $(0, \infty) \times \mathcal{P}$ with $\mathcal{P}$ as in Eq. (16). In the following, we write

$$
\mathcal{U}:=\mathbf{u}^{1} \otimes \cdots \otimes \mathbf{u}^{d} \quad \text { and } \quad \mathcal{V}:=\mathbf{v}^{1} \otimes \cdots \otimes \mathbf{v}^{d} .
$$

The Jacobian determinant of $\Phi \circ \Psi$ at $(a, b)$ is, by definition, the absolute value of the determinant of the linear map

$$
\mathrm{d}_{(a, b)}(\Phi \circ \Psi): \mathrm{T}_{\lambda}(0, \infty) \times \mathrm{T}_{\mu}(0, \infty) \times \mathrm{T}_{\left(\mathbf{u}^{1}, \ldots, \mathbf{u}^{d}\right)} \mathcal{P} \times \mathrm{T}_{\left(\mathbf{v}^{1}, \ldots, \mathbf{v}^{d}\right)} \mathcal{P} \rightarrow \mathrm{T}_{\lambda u+\mu \mathcal{V}} \mathcal{N}_{2}
$$

Consider the matrix of partial derivatives of $\Phi \circ \Psi$ with respect to the standard orthonormal basis of $\mathbb{R}^{n_{1} \times \cdots \times n_{d}}$ :

$$
Q:=[L M] \in \mathbb{R}^{\Pi \times 2 \Sigma}
$$

where

$$
L:=\left[\frac{\partial(\Phi \circ \Psi)}{\partial \mathbf{u}^{1}} \ldots \frac{\partial(\Phi \circ \Psi)}{\partial \mathbf{u}^{d}} \frac{\partial(\Phi \circ \Psi)}{\partial \mathbf{v}^{1}} \ldots \frac{\partial(\Phi \circ \Psi)}{\partial \mathbf{v}^{d}}\right] \text { and } \quad M:=\left[\frac{\partial(\Phi \circ \Psi)}{\partial \lambda} \frac{\partial(\Phi \circ \Psi)}{\partial \mu}\right]
$$


Then, the Jacobian determinant of $\Phi \circ \Psi$ at $(a, b)$ is

$$
\operatorname{Jac}(\Phi \circ \Psi)(a, b)=\sqrt{\operatorname{det}\left(Q^{T} Q\right)} .
$$

The latter is the volume of the parallelepiped spanned by the columns of $Q$. We fix notation in the next definition.

Definition 3 Let $N \geq n$ be positive integers and $U \in \mathbb{R}^{N \times n}$ be a matrix with columns $\mathbf{u}_{1}, \ldots, \mathbf{u}_{n} \in \mathbb{R}^{N}$. We denote by $\operatorname{vol}(U)$ the volume of the parallelepiped spanned by the $\mathbf{u}_{i}$ :

$$
\operatorname{vol}(U):=\sqrt{\operatorname{det}\left(U^{T} U\right)}
$$

We can now rewrite Eq. (21) as

$$
\operatorname{Jac}(\Phi \circ \Psi)(a, b)=\operatorname{vol}(Q)
$$

The reason why we write the partial derivatives of $\Phi \circ \Psi$ with respect to the standard basis of $\mathbb{R}^{n_{1} \times \cdots \times n_{d}}$ is that we get the following convenient description:

$$
M=\left[\begin{array}{ll}
\mathcal{U} & \mathcal{V}
\end{array}\right]
$$

For describing $L$, let for each $1 \leq k \leq d$,

$$
\dot{U}^{k}=\left[\dot{\mathbf{u}}_{2}^{k} \dot{\mathbf{u}}_{3}^{k} \cdots \dot{\mathbf{u}}_{n_{k}}^{k}\right] \in \mathbb{R}^{n_{k} \times\left(n_{k}-1\right)} \quad \text { and } \quad \dot{V}^{k}=\left[\dot{\mathbf{v}}_{2}^{k} \dot{\mathbf{v}}_{3}^{k} \cdots \dot{\mathbf{v}}_{n_{k}}^{k}\right] \in \mathbb{R}^{n_{k} \times\left(n_{k}-1\right)}
$$

be matrices containing as columns an ordered orthonormal basis of $\left(\mathbf{u}^{k}\right)^{\perp}=$ $\mathrm{T}_{\mathbf{u}^{k}} \mathbb{S}\left(\mathbb{R}^{n_{k}}\right)$ and $\left(\mathbf{v}^{k}\right)^{\perp}=\mathrm{T}_{\mathbf{v}^{k}} \mathbb{S}\left(\mathbb{R}^{n_{k}}\right)$, respectively. Then, by linearity and the product rule of differentiation, we have that $L=\left[\lambda L_{1} \mu L_{2}\right]$ is the block matrix consisting of 2 blocks of the form

$$
\begin{aligned}
& L_{1}=\left[L_{1}^{1} \cdots L_{1}^{d}\right] \text { with } L_{1}^{k}=\mathbf{u}^{1} \otimes \cdots \otimes \mathbf{u}^{k-1} \otimes \dot{U}^{k} \otimes \mathbf{u}^{k+1} \otimes \cdots \otimes \mathbf{u}^{d} \text { and } \\
& L_{2}=\left[L_{2}^{1} \cdots L_{2}^{d}\right] \text { with } L_{2}^{k}=\mathbf{v}^{1} \otimes \cdots \otimes \mathbf{v}^{k-1} \otimes \dot{V}^{k} \otimes \mathbf{v}^{k+1} \otimes \cdots \otimes \mathbf{v}^{d} .
\end{aligned}
$$

Both $L_{1}$ and $L_{2}$ have $\sum_{k=1}^{d}\left(n_{k}-1\right)=\Sigma-1$ columns. Note that $M$ depends only on the $\mathbf{u}^{k}$ 's and $\mathbf{v}^{k}$ 's, whereas $L$ also depends on the parameters $\lambda$ and $\mu$; we do not emphasize these dependencies in the notation.

Comparing with Breiding and Vannieuwenhoven [20, equation (5.1)], we see that the matrix $L_{1}$ has as columns an orthonormal basis for the orthogonal complement of $\mathcal{U}$ in $\mathrm{T}_{\mathcal{U}} \mathcal{S}$. Analogously, the columns of $L_{2}$ form an orthonormal basis for the orthogonal complement of $\mathcal{V}$ in $\mathrm{T}_{\mathcal{V}} \mathcal{S}$. Consequently, for $\Psi(a, b)$, Terracini's matrix from Eq. (12) can be chosen as

$$
U=\left[\begin{array}{llll}
\mathcal{U} & L_{1} & \mathcal{V} & L_{2}
\end{array}\right]
$$


This entails that

$$
\begin{aligned}
\operatorname{Jac}(\Phi \circ \Psi)(a, b) & =\sqrt{\operatorname{det}\left(\left[\lambda L_{1} \mu L_{2} \mathcal{U} \mathcal{V}\right]^{T}\left[\lambda L_{1} \mu L_{2} \mathcal{U} \mathcal{V}\right]\right)} \\
& =\lambda^{\Sigma-1} \mu^{\Sigma-1} \sqrt{\operatorname{det}\left(U^{T} U\right)} \\
& =\lambda^{\Sigma-1} \mu^{\Sigma-1} \operatorname{vol}(U)
\end{aligned}
$$

having used the notation from Definition 3 and the fact that singular values are invariant under orthogonal transformations such as permutations of columns.

\subsection{Bounding the Integral}

We are now ready to conclude the proof of Theorem 1, by showing that the expected value of the condition number of tensor rank decomposition is bounded from below by infinity.

By Eq. (12), the condition number at $\mathcal{A}=\mathcal{A}_{1}+\mathcal{A}_{2}=\Phi\left(\mathcal{A}_{1}, \mathcal{A}_{2}\right) \in \mathcal{N}_{2}$ is the inverse of the smallest singular value of the Terracini's matrix $U$ from Eq. (24). Therefore, if we plug Eqs. (24) and (25) into (18), then we get

$$
\begin{aligned}
\mathbb{E} \kappa(\mathcal{A}) & =\frac{1}{2^{2 d-1} C_{2}} \int_{((0, \infty) \times \mathcal{P})^{\times 2}} \frac{\lambda^{\Sigma-1} \mu^{\Sigma-1} \operatorname{vol}(U)}{S_{\min }(U)} e^{-\frac{\|\lambda u+\mu \mathcal{V}\|^{2}}{2}} \mathrm{~d} \lambda \mathrm{d} u \mathrm{~d} \mu \mathrm{d} v \\
& =\frac{1}{2^{2 d-1} C_{2}} \int_{((0, \infty) \times \mathcal{P})^{\times 2}} \lambda^{\Sigma-1} \mu^{\Sigma-1} q(U) e^{-\frac{\|\lambda u+\mu \mathcal{V}\|^{2}}{2}} \mathrm{~d} \lambda \mathrm{d} u \mathrm{~d} \mu \mathrm{d} v
\end{aligned}
$$

where $q(U)=\frac{\operatorname{vol}(U)}{\zeta_{\min }(U)}$ is as in Eq. (8), and

$$
u=\left(\mathbf{u}^{1}, \ldots, \mathbf{u}^{d}\right), v=\left(\mathbf{v}^{1}, \ldots, \mathbf{v}^{d}\right), u=\mathbf{u}^{1} \otimes \cdots \otimes \mathbf{u}^{d}, \text { and } \mathcal{V}=\mathbf{v}^{1} \otimes \cdots \otimes \mathbf{v}^{d}
$$

From Eq. (24), it is clear that $U$ is a function of $u$ and $v$ but is independent of $\lambda$ and $\mu$. Therefore, if we integrate first over $\lambda$ and $\mu$, then we can ignore the factor $q(U)$. In Appendix A.1, we compute this integral; the result is stated here as the next lemma.

Lemma 3 Let $\left(\mathbf{u}^{1}, \ldots, \mathbf{u}^{d}\right),\left(\mathbf{v}^{1}, \ldots, \mathbf{v}^{d}\right) \in \mathcal{P}$ be fixed. Then,

$$
\begin{aligned}
& \int_{(0, \infty)} \int_{(0, \infty)} \lambda^{\Sigma-1} \mu^{\Sigma-1} e^{-\frac{\|\lambda \mathcal{U}+\mu \mathcal{V}\|^{2}}{2}} \mathrm{~d} \lambda \mathrm{d} \mu \\
& \quad=2^{\Sigma-1} \Gamma(\Sigma) \int_{0}^{\frac{\pi}{2}} \frac{(\cos (\theta) \sin (\theta))^{\Sigma-1}}{\|\cos (\theta) \mathcal{U}+\sin (\theta) \mathcal{V}\|^{2 \Sigma}} \mathrm{d} \theta
\end{aligned}
$$

where $\mathcal{U}=\mathbf{u}^{1} \otimes \cdots \otimes \mathbf{u}^{d}$ and $\mathcal{V}=\mathbf{v}^{1} \otimes \cdots \otimes \mathbf{v}^{d}$.

The foregoing integral can be bounded from below by exploiting the next lemma, which is proved in Appendix A.2.

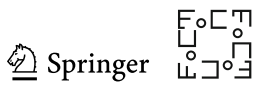


Lemma 4 Let $\mathbf{x}, \mathbf{y} \in \mathbb{S}\left(\mathbb{R}^{p}\right)$ be two unit-norm vectors and $s \geq 1$. Then, there exists a constant $k=k(p, s)$ independent of $\mathbf{x}, \mathbf{y}$ such that

$$
\int_{0}^{\frac{\pi}{2}} \frac{(\cos (\theta) \sin (\theta))^{s-1}}{\|\cos (\theta) \mathbf{x}+\sin (\theta) \mathbf{y}\|^{2 s}} \mathrm{~d} \theta \geq \frac{k}{\|\mathbf{x}+\mathbf{y}\|^{2 s-1}}
$$

Combining the foregoing lemmata and plugging the result into Eq. (26), we obtain

$$
\mathbb{E} \kappa(\mathcal{A}) \geq \frac{2^{\Sigma-1} \Gamma(\Sigma) k}{2^{2 d-1} C_{2}} \int_{\mathcal{P} \times \mathcal{P}} \frac{q(U)}{\|\mathcal{U}+\mathcal{V}\|^{2 \Sigma-1}} \mathrm{~d} u \mathrm{~d} v
$$

Next, we exploit the symmetry of the domain $\mathbb{S}\left(\mathbb{R}^{n_{1}}\right)$ by flipping the sign of $\mathbf{v}^{1}$ and, hence, of $\mathcal{V}=\mathbf{v}^{1} \otimes \cdots \otimes \mathbf{v}^{d}$. This substitution transforms $U$ into $U D$, where $D$ is a diagonal matrix with some pattern of \pm 1 on the diagonal. Since $D$ is orthogonal, $q(U)=q(U D)$, so that

$$
\mathbb{E} \kappa(\mathcal{A}) \geq \frac{2^{\Sigma-1} \Gamma(\Sigma) k}{2^{2 d-1} C_{2}} \int_{\mathcal{P} \times \mathcal{P}} \frac{q(U)}{\|\mathcal{U}-\mathcal{V}\|^{2 \Sigma-1}} \mathrm{~d} u \mathrm{~d} v .
$$

Denote this last integral by $J$, and then, it remains to show that $J=\infty$. Consider the open set

$$
D(\epsilon)=\left\{(u, v) \in \mathcal{P} \times \mathcal{P} \mid \frac{9}{10}\left\|\mathbf{u}^{1}-\mathbf{v}^{1}\right\|<\left\|\mathbf{u}^{k}-\mathbf{v}^{k}\right\|<\left\|\mathbf{u}^{1}-\mathbf{v}^{1}\right\|<\epsilon \text { for } 2 \leq k \leq d\right\} .
$$

Since $D(\epsilon)$ is open, we have

$$
J \geq \int_{D(\epsilon)} \frac{q(U)}{\|\mathcal{U}-\mathcal{V}\|^{2 \Sigma-1}} \mathrm{~d} u \mathrm{~d} v
$$

We now need two lemmata. The first one is straightforward.

Lemma 5 Let $\epsilon>0$ be sufficiently small. For all $(u, v) \in D(\epsilon)$ with $u=\left(\mathbf{u}^{1}, \ldots, \mathbf{u}^{d}\right)$ and $v=\left(\mathbf{v}^{1}, \ldots, \mathbf{v}^{d}\right)$, we have

$$
\left\|\mathbf{u}^{1}-\mathbf{v}^{1}\right\| \leq\|\mathcal{U}-\mathcal{V}\| \leq d\left\|\mathbf{u}^{1}-\mathbf{v}^{1}\right\|
$$

where $\mathcal{U}=\mathbf{u}^{1} \otimes \cdots \otimes \mathbf{u}^{d}$ and $\mathcal{V}=\mathbf{v}^{1} \otimes \cdots \otimes \mathbf{v}^{d}$.

Proof For proving the upper bound, apply the triangle inequality to the telescoping sum

$$
\sum_{i=1}^{d} \mathbf{v}^{1} \otimes \cdots \otimes \mathbf{v}^{i-1} \otimes\left(\mathbf{u}^{i}-\mathbf{v}^{i}\right) \otimes \mathbf{u}^{i+1} \otimes \cdots \otimes \mathbf{u}^{d}=\mathcal{U}-\mathcal{V}
$$


and exploit $\left\|\mathbf{u}^{k}-\mathbf{v}^{k}\right\| \leq\left\|\mathbf{u}^{1}-\mathbf{v}^{1}\right\|$ for all $k=1, \ldots, d$. The lower bound follows from

$$
\|\mathcal{U}-\mathcal{V}\|^{2}=2-2\langle\mathcal{U}, \mathcal{V}\rangle=2-2 \prod_{k=1}^{d}\left\langle\mathbf{u}^{k}, \mathbf{v}^{k}\right\rangle \geq 2-2\left\langle\mathbf{u}^{1}, \mathbf{v}^{1}\right\rangle=\left\|\mathbf{u}^{1}-\mathbf{v}^{1}\right\|^{2}
$$

having used $0<\left\langle\mathbf{u}^{k}, \mathbf{v}^{k}\right\rangle \leq 1$ for sufficiently small $\epsilon$.

The second one is the final piece of the puzzle. We prove it in Appendix A.3.

Lemma 6 For sufficiently small $\epsilon>0$, we have for all $(u, v) \in D(\epsilon)$ with $u=$ $\left(\mathbf{u}^{1}, \ldots, \mathbf{u}^{d}\right)$ and $v=\left(\mathbf{v}^{1}, \ldots, \mathbf{v}^{d}\right)$ that

$$
q(U) \geq 2^{-d / 2}\left(\frac{\left\|\mathbf{u}^{1}-\mathbf{v}^{1}\right\|}{2}\right)^{\Sigma-1}
$$

where $U$ is the matrix that depends on $u$ and $v$ as in Eq. (24) and $q$ is as in Eq. (8).

Combining Lemmas 5 and 6 with Eq. (28), we find

$$
J \geq c \int_{D(\epsilon)} \frac{1}{\left\|\mathbf{u}^{1}-\mathbf{v}^{1}\right\|^{\Sigma}} \mathrm{d} u \mathrm{~d} v
$$

where $c>0$ is some constant. Note that the integrand in this equation only depends on $\mathbf{u}^{1}$ and $\mathbf{v}^{1}$. By definition of $D(\epsilon)$, for each $2 \leq k \leq d$, and if we fix $\mathbf{u}^{k}$, the domain of integration of $\mathbf{v}^{k}$ contains the difference of two spherical caps of respective affine radii $\frac{9}{10}\left\|\mathbf{u}^{1}-\mathbf{v}^{1}\right\|$ and $\left\|\mathbf{u}^{1}-\mathbf{v}^{1}\right\|$. From Eq. (14), the volume of this difference of caps is greater than a constant times $\left\|\mathbf{u}^{1}-\mathbf{v}^{1}\right\|^{n_{j}-1}$. Therefore, if we keep $\mathbf{u}^{1}, \mathbf{v}^{1} \in \mathbb{S}\left(\mathbb{R}^{n_{1}}\right)$ constant and integrate over $\mathbf{u}^{k}, \mathbf{v}^{k} \in \mathbb{S}\left(\mathbb{R}^{n_{k}}\right), k=2, \ldots, d$, then we get

$$
J \geq c^{\prime} \int_{\substack{\mathbf{u}^{1}, \mathbf{v}^{1} \in \mathbb{S}\left(\mathbb{R}^{n}\right),\left\|\mathbf{u}^{1}-\mathbf{v}^{1}\right\| \leq \epsilon}} \frac{1}{\left\|\mathbf{u}^{1}-\mathbf{v}^{1}\right\|^{\Sigma-\left(\left(n_{2}-1\right)+\cdots+\left(n_{d}-1\right)\right)}} \mathrm{d} \mathbf{u}^{1} \mathrm{~d} \mathbf{v}^{1},
$$

where $c^{\prime}>0$ is a constant. Recall that $\Sigma=1+\sum_{k=1}^{d}\left(n_{k}-1\right)$, so that

$$
J \geq c^{\prime} \int_{\mathbf{u}^{1} \in \mathbb{S}\left(\mathbb{R}^{n_{1}}\right)} \int_{\substack{\mathbf{v}^{1} \in \mathbb{S}\left(\mathbb{R}^{n_{1}}\right),\left\|\mathbf{u}^{1}-\mathbf{v}^{1}\right\| \leq \epsilon}} \frac{1}{\left\|\mathbf{u}^{1}-\mathbf{v}^{1}\right\|^{n_{1}}} \mathrm{~d} \mathbf{v}^{1} \mathrm{~d} \mathbf{u}^{1}
$$

By rotational invariance, the inner integral does not depend on $\mathbf{u}^{1}$ and moreover for small $\epsilon$ projecting through the stereographic projection (which has a Jacobian bounded

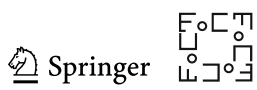


above and below by a positive constant close to its center) we conclude that, for some other constant $c^{\prime \prime}$,

$$
J \geq c^{\prime \prime} \int_{\substack{\mathbf{x} \in \mathbb{R}^{n_{1}-1},\|\mathbf{x}\| \leq \epsilon / 2}} \frac{1}{\|\mathbf{x}\|^{n_{1}}} d \mathbf{x}=c^{\prime \prime} \operatorname{vol}\left(\mathbb{S}\left(\mathbb{R}^{n_{1}-1}\right)\right) \int_{0}^{\epsilon / 2} \frac{r^{n_{1}-2}}{r^{n_{1}}} \mathrm{~d} r=\infty .
$$

This proves $J=\infty$, so that $\mathbb{E} \kappa(\mathcal{A})=\infty$ for tensors of rank bounded by 2 , constituting a proof of Theorem 1 .

\section{The Average Condition Number: From Rank 2 to Higher Ranks}

Having established that the average condition number of tensor rank decomposition of rank 2 tensors is infinite, we extend this result to higher ranks. That is, we will prove Theorem 2. As before, we abbreviate $\mathcal{S}:=\mathcal{S}_{n_{1}, \ldots, n_{d}}, \sigma_{r}:=\sigma_{r, n_{1}, \ldots, n_{d}}, \mathcal{N}_{r}:=$ $\mathcal{N}_{r, n_{1}, \ldots, n_{d}}$, and $\mathcal{M}_{r}:=\mathcal{M}_{r, n_{1}, \ldots, n_{d}}$.

We proceed with an observation that is of independent interest.

Lemma 7 Let $\mathcal{A}=\sum_{i=1}^{r} \mathcal{A}_{i}$ and $\mathcal{B}=\sum_{i=1}^{s} \mathcal{B}_{i}$ be $n_{1} \times \cdots \times n_{d}$ tensors, where the $\mathcal{A}_{i}$ and $\mathcal{B}_{i}$ are rank-1 tensors. If $\mathcal{A}+\mathcal{B} \in \sigma_{r+s ; n_{1}, \ldots, n_{d}}$ is $(r+s)$-identifiable, then we have

$$
\kappa(\mathcal{A}+\mathcal{B}) \geq \max \{\kappa(\mathcal{A}), \kappa(\mathcal{B})\}
$$

Proof First, we observe that $\mathcal{A}$ is $r$-identifiable, and $\mathcal{B}$ is $s$-identifiable. Indeed, if the tensor $\mathcal{C}=\mathcal{A}+\mathcal{B}$ is $(r+s)$-identifiable, then the unique set $C$ of cardinality $|C| \leq r$ consisting of rank-1 tensors summing to $C$ is $C=\left\{\mathcal{A}_{1}, \ldots, \mathcal{A}_{r}, \mathcal{B}_{1}, \ldots, \mathcal{B}_{s}\right\}$. If $\mathcal{A}$ had an alternative decomposition $\left\{\mathcal{A}_{1}^{\prime}, \ldots, \mathcal{A}_{r^{\prime}}^{\prime}\right\}$, potentially of a shorter length $r^{\prime} \leq r$, then $\left\{\mathcal{A}_{1}^{\prime}, \ldots, \mathcal{A}_{r^{\prime}}^{\prime}, \mathcal{B}_{1}, \ldots, \mathcal{B}_{s}\right\}$ would be an alternative decomposition of $\mathcal{C}$. Hence, $\left\{\mathcal{A}_{1}^{\prime}, \ldots, \mathcal{A}_{r^{\prime}}^{\prime}\right\}$ needs to equal $\left\{\mathcal{A}_{1}, \ldots, \mathcal{A}_{r}\right\}$, so that $\mathcal{A}$ is $r$-identifiable. By symmetry, the result for $\mathcal{B}$ follows. For all $i$, let $U_{i}$ be a matrix with orthonormal columns that span $\mathrm{T}_{\mathcal{A}_{i}} \mathcal{S}_{n_{1}, \ldots, n_{d}}$, and $V_{i}$ be a matrix with orthonormal columns that span $\mathrm{T}_{\mathcal{B}_{i}} \mathcal{S}_{n_{1}, \ldots, n_{d}}$. Consider the matrices $U=\left[U_{1}, \ldots, U_{r}\right]$ and $V=\left[V_{1}, \ldots, V_{s}\right]$. By Eq. (12), we have

$$
\kappa(\mathcal{A})=\frac{1}{\zeta_{\min }(U)}, \quad \kappa(\mathcal{B})=\frac{1}{\zeta_{\min }(V)}, \text { and } \kappa(\mathcal{A}+\mathcal{B})=\kappa(\mathcal{A}, \mathcal{B})=\frac{1}{\varsigma_{\min }\left(\left[\begin{array}{ll}
U & V
\end{array}\right)\right.} .
$$

The claim follows from standard interlacing properties of singular values; see [51, Chapter 3].

The next simple lemma is immediate.

Lemma 8 Consider the map $\phi: \sigma_{2} \times \mathcal{S}^{\times(r-2)} \rightarrow \sigma_{r},\left(\mathcal{B}, \mathcal{A}_{1}, \ldots, \mathcal{A}_{r-2}\right) \mapsto \mathcal{B}+$ $\sum_{i=1}^{r-2} \mathcal{A}_{i}$. The following holds.

1. For $r>2$, we have $\phi\left(\sigma_{2} \times \mathcal{S}^{\times(r-2)}\right)=\sigma_{r}$. 
2. Let $\mathcal{A} \in \sigma_{r}$ be r-identifiable. Then, $\left|\phi^{-1}(\mathcal{A})\right|=(r-2) ! \cdot\left(\begin{array}{l}r \\ 2\end{array}\right)$.

Finally, the next lemma is the key to Theorem 2, providing a lower bound for the Jacobian determinant of $\phi$ in a special open subset of $\sigma_{2} \times \mathcal{S}^{\times(r-2)}$. We postpone its proof to Appendix B.

Lemma 9 On top of Assumption 1 we assume that $\sigma_{r-2 ; n_{1}-2, \ldots, n_{d}-2}$ is generically complex identifiable. Then, there are constants $\mu, \epsilon, v_{1}, \ldots, v_{r-2}>0$ depending only on $r, n_{1}, \ldots, n_{d}$ with the following property: For all $\mathcal{B} \in \mathcal{N}_{2}$ there exists a tuple $\left(\mathcal{A}_{1}, \ldots, \mathcal{A}_{r-2}\right) \in \mathcal{S}^{\times(r-2)}$ with $\left\|\mathcal{A}_{i}\right\|=v_{i}$ and

$$
\inf _{\substack{\left(\mathcal{A}_{1}^{\prime}, \ldots, \mathcal{A}_{r-2}^{\prime}\right) \in \mathcal{S}^{\times(r-2)},\left\|\mathcal{A}_{i}-\mathcal{A}_{i}^{\prime}\right\|<\epsilon}} \operatorname{Jac}(\phi)\left(\mathcal{B}, \mathcal{A}_{1}^{\prime}, \ldots, \mathcal{A}_{r-2}^{\prime}\right)>\mu,
$$

where $\phi$ is as in Lemma 8.

Remark 5 Given any $\mathcal{B} \in \sigma_{2}$, by taking a sequence $\mathcal{B}^{(i)} \subseteq \mathcal{N}_{2}$ converging to $\mathcal{B}$ one can generate the corresponding sequences $\mathcal{A}_{1}^{(i)}, \ldots, \mathcal{A}_{r-2}^{(i)} \in \mathcal{S}$ from Lemma 9. Now, by compactness we can find an accumulation point $\mathcal{A}_{1}, \ldots, \mathcal{A}_{r-2} \in \mathcal{S}$. Since $\operatorname{Jac}(\phi)$ is continuous and hence uniformly continuous when restricted to a compact set, by choosing small enough $\epsilon$ we can assure that for all $\mathcal{B}^{\prime},\left\|\mathcal{B}-\mathcal{B}^{\prime}\right\| \leq \epsilon$ and for all $\mathcal{A}_{i}^{\prime}$, $\left\|\mathcal{A}_{i}-\mathcal{A}_{i}^{\prime}\right\| \leq \epsilon$, we have $\operatorname{Jac}(\phi)\left(\mathcal{B}^{\prime}, \mathcal{A}_{1}^{\prime}, \ldots, \mathcal{A}_{r-2}^{\prime}\right)>\frac{\mu}{2}$, where $\epsilon$ and $\mu$ do not depend on $\mathcal{B}$.

Now, we prove Theorem 2.

Proof of Theorem 2 Recall the surjective map $\phi: \sigma_{2} \times \mathcal{S}^{\times(r-2)} \rightarrow \sigma_{r}$ from Lemma 8 . From Theorem 1 and the fact that $\kappa(\mathcal{B})=\kappa(t \mathcal{B})$ for $t>0$, there exists a tensor $\mathcal{B} \in \sigma_{2}$ such that for every $\delta>0$ we have

$$
\int_{\left\|\mathcal{B}^{\prime}-\mathcal{B}\right\|<\delta, \mathcal{B}^{\prime} \in \mathcal{N}_{2}} \kappa\left(\mathcal{B}^{\prime}\right) \mathrm{d} \mathcal{B}^{\prime}=\infty
$$

From Lemma 9 and Remark 5, there exist tensors $\mathcal{A}_{1}, \ldots, \mathcal{A}_{r-2} \in \mathcal{S}$ such that

$$
\operatorname{Jac}(\phi)\left(\mathcal{B}^{\prime}, \mathcal{A}_{1}^{\prime}, \ldots, \mathcal{A}_{r-2}^{\prime}\right)>\frac{\mu}{2}
$$

for all $\mathcal{B}^{\prime}, \mathcal{A}_{1}^{\prime}, \ldots, \mathcal{A}_{r-2}^{\prime}$ such that $\left\|\mathcal{B}^{\prime}-\mathcal{B}\right\|<\epsilon,\left\|\mathcal{A}_{i}^{\prime}-\mathcal{A}_{i}\right\|<\epsilon$, and $\mathcal{B}^{\prime} \in \mathcal{N}_{2}$. Let $\mathcal{U} \subseteq \mathcal{N}_{2} \times \mathcal{S}^{\times r-2}$ be the set of all $\mathcal{B}^{\prime}, \mathcal{A}_{1}^{\prime}, \ldots, \mathcal{A}_{r-2}^{\prime}$ satisfying the foregoing conditions. From Lemma 7, we have

$$
\begin{aligned}
& \int_{\mathcal{U}} \operatorname{Jac}(\phi)\left(\mathcal{B}^{\prime}, \mathcal{A}_{1}^{\prime}, \ldots, \mathcal{A}_{r-2}^{\prime}\right) \kappa\left(\mathcal{B}^{\prime}+\mathcal{A}_{1}^{\prime}+\cdots+\mathcal{A}_{r-2}^{\prime}\right) \mathrm{d} \mathcal{B}^{\prime} \mathrm{d} \mathcal{A}_{1}^{\prime} \cdots \mathrm{d} \mathcal{A}_{r-2}^{\prime} \\
& \geq \int_{\mathcal{U}} \operatorname{Jac}(\phi)\left(\mathcal{B}^{\prime}, \mathcal{A}_{1}^{\prime}, \ldots, \mathcal{A}_{r-2}^{\prime}\right) \kappa\left(\mathcal{B}^{\prime}\right) \mathrm{d} \mathcal{B}^{\prime} \mathrm{d} \mathcal{A}_{1}^{\prime} \cdots \mathrm{d} \mathcal{A}_{r-2}^{\prime}=\infty
\end{aligned}
$$

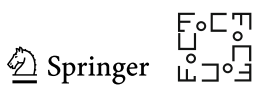


Moreover, by Lemma 9 and the inverse function theorem, by taking small enough $\epsilon$ and $\delta$ we can assume that $\phi \mid \mathcal{U}$ is a diffeomorphism onto its image $e^{4}$ and hence $\phi(\mathcal{U})$ is open. The coarea formula Eq. (15) thus applies yielding

$$
\begin{aligned}
& \int_{\mathcal{A} \in \phi(\mathcal{U})} \kappa(\mathcal{A}) \mathrm{d} \mathcal{A} \\
& =\int_{\mathcal{U}} \operatorname{Jac}(\phi)\left(\mathcal{B}^{\prime}, \mathcal{A}_{1}^{\prime}, \ldots, \mathcal{A}_{r-2}^{\prime}\right) \kappa\left(\mathcal{B}^{\prime}+\mathcal{A}_{1}^{\prime}, \ldots, \mathcal{A}_{r-2}^{\prime}\right) \mathrm{d} \mathcal{B}^{\prime} \mathrm{d} \mathcal{A}_{1}^{\prime} \cdots \mathrm{d} \mathcal{A}_{r-2}^{\prime}=\infty .
\end{aligned}
$$

The theorem follows since $\phi(\mathcal{U}) \subseteq \sigma_{r}$.

\section{The Angular Condition Number of Tensor Rank Decomposition}

In this section, we prove Theorem 3. As in the previous section, to ease notation, we abbreviate $\mathcal{M}_{2}:=\mathcal{M}_{2 ; n_{1}, \ldots, n_{d}}, \mathcal{N}_{2}:=\mathcal{N}_{2 ; n_{1}, \ldots, n_{d}}, \mathcal{S}_{2}:=\mathcal{S}_{2 ; n_{1}, \ldots, n_{d}}$, and $\sigma_{2}:=$ $\sigma_{2 ; n_{1}, \ldots, n_{d}}$.

\subsection{A Characterization of the Angular Condition Number as a Singular Value}

We first derive a formula for the angular condition number in terms of singular values, similar to the one from Eq. (12). Recall from Eq. (6) that the angular condition number for rank $r=2$ is

$$
\kappa_{\text {ang }}(\mathcal{A}):=\lim _{\epsilon \rightarrow 0} \sup _{\substack{\|\Delta \mathcal{A}\|<\epsilon, \mathcal{A}+\Delta \mathcal{A} \in \sigma_{2}}} \frac{\left\|\left((p \times p) \circ \Phi_{a}^{-1}\right)(\mathcal{A})-\left((p \times p) \circ \Phi_{a}^{-1}\right)(\mathcal{A}+\Delta \mathcal{A})\right\|}{\|\Delta \mathcal{A}\|},
$$

where $p: \mathbb{R}^{n_{1} \times \cdots \times n_{d}} \rightarrow \mathbb{S}\left(\mathbb{R}^{n_{1} \times \cdots \times n_{d}}\right)$ is the canonical projection onto the sphere and where $\Phi_{a}^{-1}$ is a local inverse of $\Phi: \mathcal{S} \times \mathcal{S} \rightarrow \sigma_{2}$ at $a \in \mathcal{S}^{\times 2}$ with $\mathcal{A}=\Phi(a)$. As before, the value of $\kappa_{\text {ang }}$ on $\sigma_{2} \backslash \mathcal{N}_{2}$ is not relevant for our analysis, so we do not specify it.

Proposition 2 Under Assumption 1 , let $\mathcal{A}=\lambda \mathbf{u}^{1} \otimes \cdots \otimes \mathbf{u}^{d}+\mu \mathbf{v}^{1} \otimes \cdots \otimes \mathbf{v}^{d} \in \mathcal{N}_{2}$, where for $1 \leq k \leq d$ we have $\mathbf{u}^{k}, \mathbf{v}^{k} \in \mathbb{S}\left(\mathbb{R}^{n_{k}}\right)$. Recall from Eq. (20) the definitions of the matrices $M$ and $L$, associated to $\mathcal{A}$. The following equality holds:

$$
\kappa_{\mathrm{ang}}(\mathcal{A})=\frac{1}{\varsigma_{\min }\left(\left(\mathrm{I}-M M^{\dagger}\right) L\right)},
$$

as far as the right-hand term is finite.

Proof By Proposition 1, any local inverse $\Phi_{a}^{-1}$ is differentiable at $\mathcal{A}=\Phi(a) \in \mathcal{N}_{2}$. The projection $p$ is also differentiable, so that

$$
\kappa_{\mathrm{ang}}(\mathcal{A})=\left\|\mathrm{d}_{\mathcal{A}}\left(p^{\times 2} \circ \Phi_{a}^{-1}\right)\right\|_{2},
$$

$\overline{4}$ This is different from $\left.\phi\right|_{\phi^{-1}(\phi(\mathcal{U}))}$ being a diffeomorphism. Indeed, that mapping is in general finite-to-one

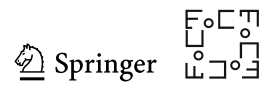


where $\|\cdot\|_{2}$ is the spectral norm from Eq. (7). We compute this norm.

Let $\dot{\mathcal{A}} \in \mathrm{T}_{\mathcal{A}} \mathcal{N}_{2}$ and $\left(\dot{\mathcal{A}}_{1}, \dot{\mathcal{A}}_{2}\right)=\mathrm{d}_{\mathcal{A}} \Phi_{a}^{-1}(\dot{\mathcal{A}})$. Then, by linearity of the derivative, we have $\dot{\mathcal{A}}=\dot{\mathcal{A}}_{1}+\dot{\mathcal{A}}_{2}$. Furthermore, for $i=1,2$, the derivative $\mathrm{d}_{\mathcal{A}_{i}} p$ is the orthogonal projection onto the orthogonal complement of $\mathcal{A}_{i}$ in $\mathbb{R}^{\Pi}$. According to this, we decompose $\dot{\mathcal{A}}_{1}$ and $\dot{\mathcal{A}}_{2}$ as

$$
\begin{aligned}
& \dot{\mathcal{A}}_{1}=\dot{\mathcal{A}}_{1}^{\perp}+\dot{\lambda} \mathcal{U}, \text { where } \mathcal{U}=\frac{\mathcal{A}_{1}}{\left\|\mathcal{A}_{1}\right\|} \text { and } \dot{\mathcal{A}}_{1}^{\perp} \in\left(\mathcal{A}_{1}\right)^{\perp}, \\
& \dot{\mathcal{A}}_{2}=\dot{\mathcal{A}}_{2}^{\perp}+\dot{\mu} \mathcal{V}, \text { where } \mathcal{V}=\frac{\mathcal{A}_{2}}{\left\|\mathcal{A}_{2}\right\|} \text { and } \dot{\mathcal{A}}_{2}^{\perp} \in\left(\mathcal{A}_{2}\right)^{\perp} .
\end{aligned}
$$

Then, we have $\mathrm{d}_{\mathcal{A}}\left(p^{\times 2} \circ \Phi_{a}^{-1}\right)(\dot{\mathcal{A}})=\left(\dot{\mathcal{A}}_{1}^{\perp} /\left\|\mathcal{A}_{1}\right\|, \dot{\mathcal{A}}_{2}^{\perp} /\left\|\mathcal{A}_{2}\right\|\right)$ and, consequently,

$$
\left\|\mathrm{d}_{\mathcal{A}}\left(p^{\times 2} \circ \Phi_{a}^{-1}\right)(\dot{\mathcal{A}})\right\|=\sqrt{\frac{\left\|\dot{\mathcal{A}}_{1}^{\perp}\right\|^{2}}{\left\|\mathcal{A}_{1}\right\|^{2}}+\frac{\left\|\dot{\mathcal{A}}_{2}^{\perp}\right\|^{2}}{\left\|\mathcal{A}_{2}\right\|^{2}}} .
$$

Recall from Eq. (20) the matrices $L=\left[\begin{array}{ll}\lambda L_{1} & \mu L_{2}\end{array}\right]$ and $M=[\mathcal{U} \mathcal{V}]$. We can find vectors $\mathbf{x}_{1}, \mathbf{x}_{2} \in \mathbb{R}^{\Sigma-1}$ with $\dot{\mathcal{A}}_{1}^{\perp}=\lambda L_{1} \mathbf{x}_{1}$ and $\dot{\mathcal{A}}_{2}^{\perp}=\mu L_{2} \mathbf{x}_{2}$, and such that $\left\|\dot{\mathcal{A}}_{1}^{\perp}\right\|=$ $\lambda\left\|\mathbf{x}_{1}\right\|$ and $\left\|\dot{\mathscr{A}}_{2}^{\perp}\right\|=\mu\left\|\mathbf{x}_{2}\right\|$. Observe that $\lambda=\left\|\mathscr{A}_{1}\right\|$ and $\mu=\left\|\mathcal{A}_{2}\right\|$. This yields

$$
\left\|\mathrm{d}_{\mathcal{A}}\left(p^{\times 2} \circ \Phi_{a}^{-1}\right)(\dot{\mathcal{A}})\right\|=\sqrt{\left\|\mathbf{x}_{1}\right\|^{2}+\left\|\mathbf{x}_{2}\right\|^{2}} .
$$

Writing

$$
\dot{\mathcal{A}}=\dot{\mathcal{A}}_{1}+\dot{\mathcal{A}}_{2}=L\left[\begin{array}{l}
\mathbf{x}_{1} \\
\mathbf{x}_{2}
\end{array}\right]+M\left[\begin{array}{l}
\dot{\lambda} \\
\dot{\mu}
\end{array}\right] \text {, we get }\left(\mathrm{I}-M M^{\dagger}\right) \dot{\mathcal{A}}=\left(\mathrm{I}-M M^{\dagger}\right) L\left[\begin{array}{l}
\mathbf{x}_{1} \\
\mathbf{x}_{2}
\end{array}\right] \text {. }
$$

Since we are assuming that $\left(\mathrm{I}-M M^{\dagger}\right) L$ is injective (for $\varsigma_{\min }\left(\left(\mathrm{I}-M M^{\dagger}\right) L\right) \neq 0$ ), it has a left inverse and we can write

$$
\left(\left(\mathrm{I}-M M^{\dagger}\right) L\right)^{\dagger}\left(\mathrm{I}-M M^{\dagger}\right) \dot{\mathcal{A}}=\left[\begin{array}{l}
\mathbf{x}_{1} \\
\mathbf{x}_{2}
\end{array}\right] .
$$

Combining Eqs. (30) and (31), we see that

$$
\begin{aligned}
\kappa_{\text {ang }}(\mathcal{A}) & =\left\|\left(\left(\mathrm{I}-M M^{\dagger}\right) L\right)^{\dagger}\left(\mathrm{I}-M M^{\dagger}\right)\right\|_{2} \\
& =\left\|\left(\left(\mathrm{I}-M M^{\dagger}\right) L\right)^{\dagger}\right\|_{2} \\
& =\varsigma_{\min }\left(\left(\mathrm{I}-M M^{\dagger}\right) L\right)^{-1},
\end{aligned}
$$

the second equality from $(P L)^{\dagger} P=(P L)^{\dagger}$, which is a basic property of the MoorePenrose pseudo-inverse holding for any orthogonal projector $P$. This finishes the proof.

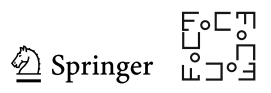




\subsection{Proof of Theorem 3}

Now comes the actual proof of Theorem 3. Proceeding in exactly the same way as in Sect. 3.1 and using Proposition 2, we get

$$
\mathbb{E} \kappa_{\text {ang }}(\mathcal{A})=\frac{1}{2^{2 d-1} C_{2}} \int_{((0, \infty) \times \mathcal{P})^{\times 2}} \frac{\operatorname{vol}(Q) e^{-\frac{\|\lambda \mathcal{U}+\mu \mathcal{V}\|^{2}}{2}}}{\zeta_{\min }\left(\left(\mathrm{I}-M M^{\dagger}\right) L\right)} \mathrm{d} \lambda \mathrm{d} u \mathrm{~d} \mu \mathrm{d} v
$$

where $C_{2}=C_{2 ; n_{1}, \ldots, n_{d}}$ is as in Definition $1, \mathcal{P}$ is as in Eq. (16), $Q=[L M]$ is as in Eq. (19), the volume vol is as in Definition 3, and

$u=\left(\mathbf{u}^{1}, \ldots, \mathbf{u}^{d}\right), v=\left(\mathbf{v}^{1}, \ldots, \mathbf{v}^{d}\right), \quad \mathcal{U}=\mathbf{u}^{1} \otimes \cdots \otimes \mathbf{u}^{d}, \quad$ and $\mathcal{V}=\mathbf{v}^{1} \otimes \cdots \otimes \mathbf{v}^{d}$,

is as in Eq. (27), so that $\mathcal{A}=\lambda \mathcal{U}+\mu \mathcal{V}$. Next, we relate $\operatorname{vol}(Q)$ to the volume of $\left(\mathrm{I}-M M^{\dagger}\right) L$.

Lemma 10 We have $\operatorname{vol}(Q)=\operatorname{vol}(M) \operatorname{vol}\left(\left(\mathrm{I}-M M^{\dagger}\right) L\right)$.

Proof Let $Q^{\perp}$ be a matrix whose columns contain an orthonormal basis for the orthogonal complement of the column span of $Q$. Then, from the definition,

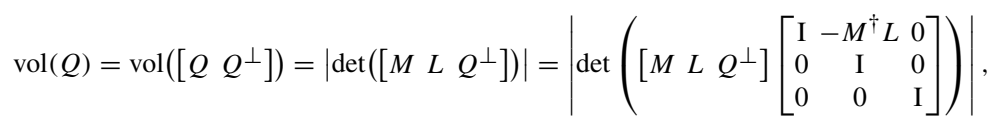

where in the last step we just multiplied by a matrix whose determinant is 1 . Performing the inner multiplication, we then get

$$
\operatorname{vol}(Q)=\left|\operatorname{det}\left(\left[M\left(\mathrm{I}-M M^{\dagger}\right) L Q^{\perp}\right]\right)\right|=\operatorname{vol}\left(\left[M\left(\mathrm{I}-M M^{\dagger}\right) L\right]\right) .
$$

These two blocks are mutually orthogonal, since $\left(\mathrm{I}-M M^{\dagger}\right)$ is the projection on the orthogonal complement of the span of $M$, and hence, the volume is the product of the volumes corresponding to each block. The assertion follows.

We use Lemma 10 to rewrite Eq. (32) as

$$
\begin{aligned}
& \mathbb{E} \kappa_{\text {ang }}(\mathcal{A})=\frac{1}{2^{2 d-1} C_{2}} \int_{((0, \infty) \times \mathcal{P})^{\times 2}} \frac{\operatorname{vol}(M) \operatorname{vol}\left(\left(\mathrm{I}-M M^{\dagger}\right) L\right)}{S_{\min }\left(\left(\mathrm{I}-M M^{\dagger}\right) L\right)} e^{-\frac{\|\lambda \mathcal{U}+\mu \mathcal{V}\|^{2}}{2}} \mathrm{~d} \lambda \mathrm{d} u \mathrm{~d} \mu \mathrm{d} v \\
& =\frac{1}{2^{2 d-1} C_{2}} \int_{((0, \infty) \times \mathcal{P})^{\times 2}} \operatorname{vol}(M) q\left(\left(\mathrm{I}-M M^{\dagger}\right) L\right) e^{-\frac{\|\lambda u+\mu \mathcal{V}\|^{2}}{2}} \mathrm{~d} \lambda \mathrm{d} u \mathrm{~d} \mu \mathrm{d} v,
\end{aligned}
$$

where $q$ is as in Eq. (8). Recall from Eq. (22) that $M$ is independent of $\lambda$ and $\mu$. We first compute the integral over $\lambda, \mu$ using the next lemma. We prove the lemma in Appendix C.1.

Lemma 11 Let $L_{1}, L_{2}$ be the matrices defined as in Eq. (23), such that $L=$ $\left[\lambda L_{1} \mu L_{2}\right]$. Let

$$
J_{\text {inner }}=\int_{(0, \infty)^{2}} q\left(\left(\mathrm{I}-M M^{\dagger}\right) L\right) e^{-\frac{\|\lambda U+\mu \mathcal{V}\|^{2}}{2}} \mathrm{~d} \lambda \mathrm{d} \mu
$$

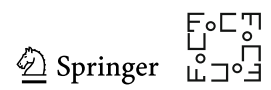


Then,

$$
J_{\text {inner }}=2^{\frac{2 \Sigma-3}{2}} \Gamma\left(\frac{2 \Sigma-1}{2}\right) \int_{0}^{\frac{\pi}{2}} \frac{q\left(\left(\mathrm{I}-M M^{\dagger}\right)\left[\cos (\theta) L_{1} \sin (\theta) L_{2}\right]\right)}{\|\cos (\theta) \mathcal{U}+\sin (\theta) \mathcal{V}\|^{2 \Sigma-1}} \mathrm{~d} \theta
$$

Inserting the results from this lemma into Eq. (33), we get

$$
\mathbb{E} \kappa_{\mathrm{ang}}(\mathcal{A})=\frac{2^{\frac{2 \Sigma-1}{2}-2 d} \Gamma\left(\frac{2 \Sigma-1}{2}\right)}{C_{2}} J_{\text {outer }}
$$

where

$$
J_{\text {outer }}:=\int_{\mathcal{P}^{\times 2}} \int_{0}^{\frac{\pi}{2}} \frac{\operatorname{vol}(M) q\left(\left(\mathrm{I}-M M^{\dagger}\right)\left[\cos (\theta) L_{1} \sin (\theta) L_{2}\right]\right)}{\|\cos (\theta) \mathcal{U}+\sin (\theta) \mathcal{V}\|^{2 \Sigma-1}} \mathrm{~d} \theta \mathrm{d} u \mathrm{~d} v .
$$

In the remaining part of this section, we show that $J_{\text {outer }}$ is bounded by a constant, which would conclude the proof. We do this by giving a sequence of upper bounds. We have no hope of providing sharp bounds, so rather than keeping track of all the constants, we will exploit the following definition for streamlining the proof.

Definition 4 For $A, B \in[0, \infty]$, we will write $A \preceq B$ if $B \in \mathbb{R}$ implies $A \in \mathbb{R}$. That is, $A \preceq B$ is an equivalent statement to " $B<\infty \Rightarrow A<\infty$."

First, note that $\operatorname{vol}(M)=\sqrt{1-\langle\mathcal{U}, \mathcal{V}\rangle^{2}}$, so that

$$
J_{\text {outer }}=\int_{\mathcal{P}^{\times 2}} \int_{0}^{\frac{\pi}{2}} \frac{\sqrt{1-\langle\mathcal{U}, \mathcal{V}\rangle^{2}} q\left(\left(\mathrm{I}-M M^{\dagger}\right)\left[\cos (\theta) L_{1} \sin (\theta) L_{2}\right]\right)}{\|\cos (\theta) \mathcal{U}+\sin (\theta) \mathcal{V}\|^{2 \Sigma-1}} \mathrm{~d} \theta \mathrm{d} u \mathrm{~d} v
$$

Next, we exploit the symmetry of $\mathbb{S}\left(\mathbb{R}^{n_{1}}\right)$ and transform $\mathbf{v}^{1} \mapsto-\mathbf{v}^{1}$. This transformation flips the sign of $\mathcal{V}$, but the value of $q$ is not affected. Indeed, the matrix I $-M M^{\dagger}$ still projects onto $\operatorname{span}(\mathcal{U}, \mathcal{V})^{\perp}=\operatorname{span}(\mathcal{U},-\mathcal{V})^{\perp}$, and $L_{2}$ is transformed into $L_{2} D$, where $D$ is a diagonal matrix with some pattern of \pm 1 on the diagonal. Since $\left[{ }^{I}{ }_{D}\right]$ is an orthogonal transformation, the singular values do not change. Thus, we obtain

$$
J_{\text {outer }}=\int_{\mathcal{P}^{\times 2}} \int_{0}^{\frac{\pi}{2}} \frac{\sqrt{1-\langle\mathcal{U}, \mathcal{V}\rangle^{2}} q\left(\left(\mathrm{I}-M M^{\dagger}\right)\left[\cos (\theta) L_{1} \sin (\theta) L_{2}\right]\right)}{\|\cos (\theta) \mathcal{U}-\sin (\theta) \mathcal{V}\|^{2 \Sigma-1}} \mathrm{~d} \theta \mathrm{d} u \mathrm{~d} v .
$$

The next lemma is proved in Appendix C.2.

Lemma 12 Let $\theta \in\left[0, \frac{\pi}{2}\right]$ and fix $\theta$, u and $v$. There is a constant $K>0$, depending only on $n_{1}, \ldots, n_{d}$ and $d$, such that

$$
q\left(\left(\mathrm{I}-M M^{\dagger}\right)\left[\cos (\theta) L_{1} \sin (\theta) L_{2}\right]\right) \leq K\|\mathcal{U}-\mathcal{V}\|^{\Sigma-1}
$$

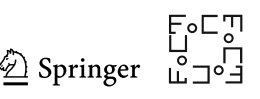


The lemma implies

$$
J_{\text {outer }} \preceq \int_{\mathcal{P}^{\times 2}} \int_{0}^{\frac{\pi}{2}} \frac{\sqrt{1-\langle\mathcal{U}, \mathcal{V}\rangle^{2}}\|\mathcal{U}-\mathcal{V}\|^{\Sigma-1}}{\|\cos (\theta) \mathcal{U}-\sin (\theta) \mathcal{V}\|^{2 \Sigma-1}} \mathrm{~d} \theta \mathrm{d} u \mathrm{~d} v .
$$

For bounding the integral over $\theta$, we need the next lemma, which we prove in Appendix C.3.

Lemma 13 Let $a>1, p \geq 1$. There exists a constant $K>0$, depending only on $a$, such that for any unit vectors $\mathbf{x}, \mathbf{y} \in \mathbb{S}\left(\mathbb{R}^{p}\right), \mathbf{x} \neq \mathbf{y}$, we have

$$
\int_{0}^{\frac{\pi}{2}} \frac{1}{\|\cos (\theta) \mathbf{x}-\sin (\theta) \mathbf{y}\|^{a}} \mathrm{~d} \theta \leq \frac{K}{\|\mathbf{x}-\mathbf{y}\|^{a-1}} .
$$

Applying this lemma to Eq. (35), we obtain

$$
J_{\text {outer }} \preceq \int_{\mathcal{P}^{\times 2}} \frac{\sqrt{1-\langle\mathcal{U}, \mathcal{V}\rangle^{2}}}{\|\mathcal{U}-\mathcal{V}\|^{\Sigma-1}} \mathrm{~d} u \mathrm{~d} v=\int_{\mathcal{P}^{\times 2}} \frac{\sqrt{1-\langle\mathcal{U}, \mathcal{V}\rangle} \sqrt{1+\langle\mathcal{U}, \mathcal{V}\rangle}}{\|\mathcal{U}-\mathcal{V}\|^{\Sigma-1}} \mathrm{~d} u \mathrm{~d} v .
$$

Writing $\|\mathcal{U}-\mathcal{V}\|=\sqrt{2} \sqrt{1-\langle\mathcal{U}, \mathcal{V}\rangle}$, we arrive at

$$
J_{\text {outer }} \preceq \int_{\mathcal{P}^{\times 2}} \frac{\sqrt{1+\langle\mathcal{U}, \mathcal{V}\rangle}}{\sqrt{1-\langle\mathcal{U}, \mathcal{V}\rangle}^{\Sigma-2}} \mathrm{~d} u \mathrm{~d} v \preceq \int_{\mathcal{P}^{\times 2}} \frac{1}{\sqrt{1-\langle\mathcal{U}, \mathcal{V}\rangle}^{\Sigma-2}} \mathrm{~d} u \mathrm{~d} v .
$$

By orthogonal invariance, we may fix $\mathbf{u}^{k} \in \mathbb{S}\left(\mathbb{R}^{n_{k}}\right)$ to be $\mathbf{u}^{k}=(1,0, \ldots, 0)$, and integrate the constant function 1 over one copy of $\mathbb{S}\left(\mathbb{R}^{n_{1}}\right) \times \cdots \times \mathbb{S}\left(\mathbb{R}^{n_{d}}\right)$. Ignoring the product of volumes $\prod_{k=1}^{d} \operatorname{vol}\left(\mathbb{S}\left(\mathbb{R}^{n_{k}}\right)\right)$ we have

$$
J_{\text {outer }} \preceq \int_{\mathbb{S}_{\left(\mathbb{R}^{n_{1}}\right) \times \cdots \times \mathbb{S}\left(\mathbb{R}^{n} d\right)}} \frac{1}{{\sqrt{1-\left(\mathbf{v}^{1}\right)_{1} \cdots\left(\mathbf{v}^{d}\right)_{1}}}^{\Sigma-2}} \mathrm{~d} v .
$$

Now, this spherical integral is particularly simple because the integrand depends uniquely on one of the components of each vector. One can thus transform each integral in a sphere into an integral in an interval (see, for example, [10, Lemma 1]) getting:

$$
J_{\text {outer }} \preceq \int_{t_{1}, \ldots, t_{d} \in[-1,1]} \frac{\left(1-t_{1}^{2}\right)^{\frac{n_{1}-1}{2}-1} \cdots\left(1-t_{d}^{2}\right)^{\frac{n_{d}-1}{2}-1}}{{\sqrt{1-t_{1} \cdots t_{d}}}^{\Sigma-2}} \mathrm{~d} t_{1} \cdots \mathrm{d} t_{d} .
$$

For this last integral, we consider the partition of the cube $[-1,1]^{d}$ into $2^{d}$ pieces corresponding to the different signs of the coordinates. In the pieces where the number of negative coordinates is odd, the denominator of the integrand is bounded below by 1 and thus the whole integrand is also bounded above by 1 . Hence, it suffices to check that the integral in the rest of the pieces is bounded. Assume now that $t_{i_{1}}, \ldots, t_{i_{k}}$ with 
$k \geq 2$ even are the negative coordinates in some particular piece of the partition. The mapping that leaves all coordinates fixed but maps $t_{i_{k-1}} \mapsto-t_{i_{k-1}}$ and $t_{i_{k}} \mapsto-t_{i_{k}}$ preserves the integrand and moves the domain to another piece of the partition with $k-2$ negative coordinates. This process can then be repeated until none of the coordinates is negative. All in one, we have

$$
J_{\text {outer }} \preceq \int_{t_{1}, \ldots, t_{d} \in[0,1]} \frac{\left(1-t_{1}^{2}\right)^{\frac{n_{1}-1}{2}-1} \cdots\left(1-t_{d}^{2}\right)^{\frac{n_{d}-1}{2}-1}}{{\sqrt{1-t_{1} \cdots t_{d}}}^{\Sigma-2}} \mathrm{~d} t_{1} \cdots \mathrm{d} t_{d} .
$$

The change of variables $t_{k}=\cos \left(\theta_{k}\right)$ for $1 \leq k \leq d$ converts this last integral into

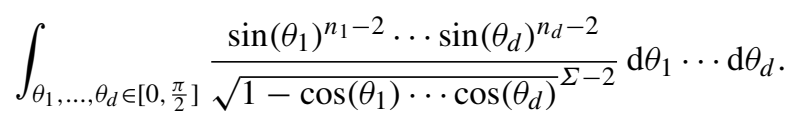

The next lemma is proved in Appendix C.4.

Lemma 14 Let $d \geq 1$ and $\theta_{1}, \ldots, \theta_{d} \in\left[0, \frac{\pi}{2}\right]$. Then, $\cos \left(\theta_{1}\right) \cdots \cos \left(\theta_{d}\right) \leq 1-$ $\frac{\theta_{1}^{2}+\cdots+\theta_{d}^{2}}{7 d}$.

Using the lemma and the inequality $\sin (\theta)<\theta$ on $0 \leq \theta \leq \frac{\pi}{2}$, we find that the integral in Eq. (36) is bounded by a constant times the following integral:

$$
\int_{\theta_{1}, \ldots, \theta_{d} \in\left[0, \frac{\pi}{2}\right]} \frac{\theta_{1}^{n_{1}-2} \cdots \theta_{d}^{n_{d}-2}}{{\sqrt{\theta_{1}^{2}+\cdots+\theta_{d}^{2}}}^{\Sigma-2}} \mathrm{~d} \theta_{1} \cdots \mathrm{d} \theta_{d} .
$$

Changing the name of the variables to $x_{1}, \ldots, x_{d}$ and integrating over the $d$ dimensional ball of radius $\frac{\pi}{2} \sqrt{d}$, which contains the domain $\left[0, \frac{\pi}{2}\right]^{d}$, we get a new upper bound for the last integral, which implies

$$
J_{\text {outer }} \preceq \int_{x_{1}^{2}+\cdots+x_{d}^{2} \leq \frac{\pi}{4} d^{4}} \frac{x_{1}^{n_{1}-2} \cdots x_{d}^{n_{d}-2}}{{\sqrt{x_{1}^{2}+\cdots+x_{d}^{2}}}^{\Sigma-2}} \mathrm{~d} x_{1} \cdots \mathrm{d} x_{d} .
$$

Recall that $\Sigma=1+\sum_{j=1}^{d}\left(n_{j}-1\right)$. By passing to polar coordinates, we get

$$
\begin{aligned}
J_{\text {outer }} & \preceq \int_{x \in \mathbb{S}\left(\mathbb{R}^{d}\right)} x_{1}^{n_{1}-2} \cdots x_{d}^{n_{d}-2} \int_{0}^{\frac{\pi \sqrt{d}}{2}} \frac{\rho^{d-1+\sum_{j=1}^{d}\left(n_{j}-2\right)}}{\rho^{-1+\sum_{j=1}^{d}\left(n_{j}-1\right)} \mathrm{d} \rho \mathrm{d} x_{1} \cdots \mathrm{d} x_{d}} \\
& \leq \operatorname{vol}\left(\mathbb{S}_{\left.\left(\mathbb{R}^{d}\right)\right)} \int_{0}^{\frac{\pi \sqrt{d}}{2}} 1=\operatorname{vol}\left(\mathbb{S}^{d}\left(\mathbb{R}^{d}\right)\right) \frac{\pi \sqrt{d}}{2}<\infty .\right.
\end{aligned}
$$

This shows $J_{\text {outer }}<\infty$ implying $\mathbb{E} \kappa_{\text {ang }}(\mathcal{A})<\infty$, finishing the proof of Theorem 3 .

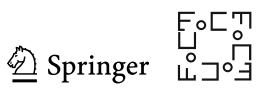




\section{Other Random Tensors: Proof of Theorem 4}

We demonstrate how our main results can be extended to many other distributions as well.

Consider the first item of Theorem 4 . We assume that $\mathcal{A} \in \sigma_{r ; n_{1}, \ldots, n_{d}}$ has the density $\hat{\rho}$ and that there exists positive constants $c_{1}, c_{2}$ such that $c_{1} \leq \frac{\hat{\rho}}{\rho} \leq c_{2}$, where $\rho$ is the density of a GIT. Then, for any measurable function $f(\mathcal{A})$ we have

$$
\mathbb{E}_{\mathcal{A} \sim \hat{\rho}} f(\mathcal{A})=\int_{\sigma_{r ; n_{1}, \ldots, n_{d}}} f(\mathcal{A}) \hat{\rho}(\mathcal{A}) \mathrm{d} \mathcal{A} \geq c_{1} \int_{\sigma_{r ; n_{1}, \ldots, n_{d}}} f(\mathcal{A}) \rho(\mathcal{A}) \mathrm{d} \mathcal{A}=c_{1} \mathbb{E}_{\mathscr{A} \sim \rho} \quad f(\mathcal{A})
$$

and

$$
\mathbb{E}_{\mathcal{A} \sim \hat{\rho}} f(\mathcal{A})=\int_{\sigma_{r ; n_{1}, \ldots, n_{d}}} f(\mathcal{A}) \hat{\rho}(\mathcal{A}) \mathrm{d} \mathcal{A} \leq c_{2} \int_{\sigma_{r ; n_{1}, \ldots, n_{d}}} f(\mathcal{A}) \rho(\mathcal{A}) \mathrm{d} \mathcal{A}=c_{2} \mathbb{E}_{\mathcal{A} \sim \rho} \quad f(\mathcal{A})
$$

Thus, $\mathbb{E}_{\mathcal{A} \sim \hat{\rho}} f(\mathcal{A})=\infty$ if and only if $\mathbb{E}_{\mathcal{A} \sim \rho} f(\mathcal{A})=\infty$. Replacing $f$ by $\kappa$ and $\kappa_{\text {ang }}$ proves the first part of Theorem 4.

By Breiding and Vannieuwenhoven [20, Proposition 4.4], $\kappa$ is invariant under multiplication of $\mathcal{A}$ by a scalar. Therefore, the expected value of $\kappa$ for the Gaussian is equal to the expected value when $\mathcal{A}$ is chosen uniformly in the unit ball, and also when $\mathcal{A}$ is chosen uniformly in the unit sphere of the space of tensors. Namely, we have (see, e.g., [28, Section 2.2.4])

$$
\mathbb{E}_{\mathcal{A} \in \sigma_{r ; n_{1}, \ldots, n_{d}}:\|\mathcal{A}\| \leq 1} \kappa(\mathcal{A})=\mathbb{E}_{\mathcal{A} \in \sigma_{r ; n_{1}, \ldots, n_{d}}:\|\mathcal{A}\|=1} \kappa(\mathcal{A})=\mathbb{E}_{\mathcal{A} \text { a GIT in } \sigma_{r ; n_{1}, \ldots, n_{d}}} \kappa(\mathcal{A})
$$

This proves the second and third item of Theorem 4 for $\kappa$.

For $\kappa_{\text {ang }}$, we need the following lemma.

Lemma 15 If $\mathcal{A} \in \sigma_{r ; n_{1}, \ldots, n_{d}}$ is an $r$-nice tensor, then $\kappa_{\mathrm{ang}}(t \mathcal{A})=\kappa_{\mathrm{ang}}(\mathcal{A}) / t$ for all $t>0$.

Proof Since $\mathcal{A}$ is $r$-nice, we have $\kappa_{\text {ang }}(\mathcal{A})=\left\|\mathrm{d}_{\mathcal{A}}\left(p^{\times r} \circ \Phi_{a}^{-1}\right)\right\|$. Similar as for Eq. (29), we can show $\left\|\mathrm{d}_{\mathcal{A}}\left(p^{\times r} \circ \Phi_{a}^{-1}\right)(\dot{\mathcal{A}})\right\|=\sqrt{\sum_{i=1}^{r}\left\|\mathcal{A}_{i}\right\|^{-2}\left\|\mathrm{~d}_{\mathcal{A}_{i}} p \dot{\mathcal{A}}_{i}\right\|^{2}}$, where $\mathcal{A}=\mathcal{A}_{1}+\cdots+\mathcal{A}_{r}$ is the CPD of $\mathcal{A}$ and $\dot{\mathcal{A}}=\dot{\mathcal{A}}_{1}+\cdots+\dot{\mathcal{A}}_{r}$ is the corresponding decomposition the tangent vector. The derivative $\mathrm{d}_{\mathcal{A}_{i}} p$ is the orthogonal projection onto $\mathcal{A}_{i}^{\perp}$ and independent of scaling. Moreover, $\sigma_{r ; n_{1}, \ldots, n_{d}}$ is a cone and so $\mathrm{T}_{\mathcal{A}} \sigma_{r ; n_{1}, \ldots, n_{d}}$ can be identified with $\mathrm{T}_{t \mathcal{A}} \sigma_{r ; n_{1}, \ldots, n_{d}}$. This shows that after scaling the tensor $\mathcal{A}$ we get $\left\|\mathrm{d}_{t \mathcal{A}}\left(p^{\times r} \circ \Phi_{a}^{-1}\right)(\dot{\mathcal{A}})\right\|=t^{-1}\left\|\mathrm{~d}_{\mathcal{A}}\left(p^{\times r} \circ \Phi_{a}^{-1}\right)(\dot{\mathcal{A}})\right\|$ and hence $\kappa_{\text {ang }}(t \mathcal{A})=\kappa_{\text {ang }}(\mathcal{A}) / t$.

Now, we can prove the rest of Theorem 4. Recall from Definition 1 that the density of a GIT on $\sigma_{r ; n_{1}, \ldots, n_{d}}$ is $\rho(\mathcal{A}):=\left(C_{r ; n_{1}, \ldots, n_{d}}\right)^{-1} e^{-\frac{\|\mathscr{A}\|^{2}}{2}}$, where $C_{r ; n_{1}, \ldots, n_{d}}=$ $\int_{\sigma_{r ; n_{1}, \ldots, n_{d}}} e^{-\frac{\|\mathcal{A}\|^{2}}{2}} \mathrm{~d} \mathcal{A}$. Since our results for $\kappa_{\text {ang }}$ are for rank-2 tensors, we put $r=2$ in the following. We also abbreviate $\sigma_{2}:=\sigma_{2 ; n_{1}, \ldots, n_{d}}$ and $C_{2}:=C_{2 ; n_{1}, \ldots, n_{d}}$. Then, using Lemma 15 we can integrate in polar coordinates to obtain 


$$
\begin{aligned}
\mathbb{E}_{\mathcal{A} \text { a GIT in } \sigma_{2}} \kappa_{\mathrm{ang}}(\mathcal{A}) & =\frac{1}{C_{2}} \int_{\mathcal{A} \in \sigma_{2}} \kappa_{\mathrm{ang}}(\mathcal{A}) e^{-\frac{\|\mathscr{A}\|^{2}}{2}} \mathrm{~d} \mathcal{A} \\
& =\frac{1}{C_{2}} \int_{0}^{\infty} e^{-\frac{t^{2}}{2}} \int_{\mathcal{A} \in \sigma_{2}:\|\mathcal{A}\|=t} \kappa_{\mathrm{ang}}(\mathcal{A}) \mathrm{d} \mathcal{A} \mathrm{d} t \\
& =\frac{1}{C_{2}} \int_{0}^{\infty} e^{-\frac{t^{2}}{2}} \int_{\mathcal{A} \in \mathbb{S}\left(\sigma_{2}\right)} t^{2 \Sigma-1} \kappa_{\mathrm{ang}}(t \mathcal{A}) \mathrm{d} \mathcal{A} \mathrm{d} t \\
& =\frac{1}{C_{r}} \int_{0}^{\infty} t^{2 \Sigma-2} e^{-\frac{t^{2}}{2}} \mathrm{~d} t \int_{\mathcal{A} \in \mathbb{S}\left(\sigma_{2}\right)} \kappa_{\mathrm{ang}}(\mathcal{A}) \mathrm{d} \mathcal{A} .
\end{aligned}
$$

It follows immediately that the last integral is finite, proving that a randomly chosen $\mathcal{A} \in \mathbb{S}\left(\sigma_{2}\right)$ has finite expected $\kappa_{\text {ang. }}$. Finally, if $\mathcal{A}$ is chosen randomly in the unit ball in $\sigma_{2}$, the same argument shows that the expected value is again finite:

$$
\int_{\mathcal{A} \in \sigma_{r ; n_{1}, \ldots, n_{d}}:\|\mathcal{A}\| \leq 1} \kappa_{\text {ang }}(\mathcal{A}) \mathrm{d} \mathcal{A}=\int_{0}^{1} t^{2 \Sigma-2} \int_{\mathcal{A} \in \mathbb{S}\left(\sigma_{2}\right)} \kappa_{\text {ang }}(\mathcal{A}) \mathrm{d} \mathcal{A} \mathrm{d} t<\infty .
$$

This finishes the proof of Theorem 4.

\section{Numerical Experiments}

Having proved that the expected value of the condition number is infinite in most cases, we provide further computational evidence in support of Conjecture 1. To this end, a natural idea is to perform Monte Carlo experiments in a few of the unknown cases as in [23].

Sampling GITs is hard in practice, as the defining polynomial equalities and inequalities of the semialgebraic set $\sigma_{r}=\sigma_{r ; n_{1}, \ldots, n_{d}}$ of tensors of rank bounded by $r$ are not known in the literature. ${ }^{5}$ Nevertheless, there are a few cases that we can treat numerically. If $r=\frac{\Pi}{\Sigma}$ and the algebraic closure $\overline{\sigma_{r}}(\mathbb{C})$ has $\operatorname{dim} \overline{\sigma_{r}}(\mathbb{C})=\Pi$, a so-called perfect tensor space, then $\sigma_{r}$ is an open subset of the ambient $\mathbb{R}^{n_{1} \times \cdots \times n_{d}}$; see, e.g., [15,57].

From Remark 2, we can sample from the density $\rho$ on $\sigma_{r}$ via an acceptance-rejection method: Randomly sample tensors $\mathcal{A}$ from the density $e^{-\frac{\|\mathcal{A}\|^{2}}{2}}$ on $\mathbb{R}^{n_{1} \times \cdots \times n_{d}}$ until we find one that belongs to $\sigma_{r}$. While this scheme will yield tensors distributed according to the density $\rho$ on $\sigma_{r}$, it does not yield Gaussian identifiable tensors in general. The reason is that most perfect tensor spaces are not (expected to be) generically $r$-identifiable [47]. Fortunately, there are a few known exceptions: matrix pencils $\left(\mathbb{R}^{n \times n \times 2}\right.$ for all $n \geq 2$ ), $\mathbb{R}^{5 \times 4 \times 3}$ and $\mathbb{R}^{3 \times 2 \times 2 \times 2}$ are proved to be generically complex $r$-identifiable for $r=\frac{\Pi}{\Sigma}$. By applying the acceptance-rejection method to these spaces, every sampled tensor is a GIT with probability 1.

For numerically checking, if a random tensor $\mathcal{A} \in \mathbb{R}^{n_{1} \times \cdots \times n_{d}}$ in a perfect tensor space lies in $\sigma_{r}$ with $r=\frac{\Pi}{\Sigma}$, we apply a homotopy continuation method to the square

\footnotetext{
${ }^{5}$ See [57, Chapter 7] and the references therein for some results on equations of the algebraic closure of $\sigma_{r}$.

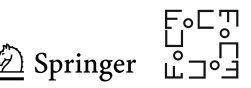


system of $\Pi$ equations

$$
\mathcal{A}-\sum_{i=1}^{r} \mathbf{a}_{i}^{1} \otimes\left[\begin{array}{c}
1 \\
\mathbf{a}_{i}^{2}
\end{array}\right] \otimes \cdots \otimes\left[\begin{array}{c}
1 \\
\mathbf{a}_{i}^{d}
\end{array}\right]=0
$$

where the $\Pi=r \Sigma$ entries of the $\mathbf{a}_{i}^{k}$, s are treated as variables, and the $n_{1} \times \cdots \times n_{d}$ tensor $\mathcal{A}$ is the tensor to decompose. We generate a start system with one solution to track by randomly sampling the entries of the vectors $\mathbf{a}_{i}^{k}$ i.i.d. from a real standard Gaussian distribution and then constructing the corresponding tensor $\mathcal{A}_{0}$. Since $r=\frac{\Pi}{\Sigma}$ is the so-called generic rank of tensors in perfect tensor spaces $\mathbb{C}^{n_{1} \times \cdots \times n_{d}}$, the above system has at least one complex solution with probability 1 as well. If we consider complex $r$-identifiable perfect tensor spaces at the generic rank, we can thus determine if $\mathcal{A} \in \sigma_{r}$ by solving the square system and checking whether the unique solution is real. Assuming that we use a certified homotopy method such as alphaCertified [48], this approach will correctly classify $\mathcal{A}$ with probability 1 , thus not impacting the overall distribution produced by the acceptance-rejection scheme.

We implemented the above scheme in Julia 1.0.3 using version 0.4.3 of the package HomotopyContinuation.j1 [19], employing the solve function with default parameter settings. We deem a solution real if the norm of the imaginary part is less than $10^{-8}$. Note that this package does not offer certified tracking; however, the failure rate observed in our experiments was very low, namely $0.0512498 \%$ - see Table 1 . For this reason, we are convinced that the distribution produced by the acceptance-rejection scheme is very close to the true distribution.

We performed the following experiment for estimating the distribution of the condition numbers of GITs of generically complex $r$-identifiable tensors in perfect tensor spaces with $r=\frac{\Pi}{\Sigma}$, the complex generic rank. As explained above, we randomly sampled an element $\mathcal{A}$ of $\mathbb{R}^{n_{1} \times \cdots \times n_{d}}$ from the density $e^{-\frac{\|\mathcal{A}\|^{2}}{2}}$ by choosing its entries i.i.d. standard normally distributed. Then, we generated one random starting system and applied the solve function from HomotopyContinuation.jl for tracking the starting solution $\mathcal{A}_{0}$ to the target $\mathcal{A}$. If the final solution of the square system was real, we recorded both the regular and angular condition numbers at the CPD of $\mathcal{A}$ computed via homotopy continuation. These computations were performed in parallel using 20 computational threads until 100,000 finite, nonsingular, real solutions and corresponding condition numbers were obtained. This experiment was performed on a computer system consisting of 2 Intel Xeon E5-2697 v3 CPUs with 12 cores clocked at $2.6 \mathrm{GHz}$ and $128 \mathrm{~GB}$ main memory. Information about the sampling process via the acceptancerejection method is summarized in Table 1, and Fig. 1 visualizes the complementary cumulative distribution functions of the regular and angular condition numbers.

In Table 1, the total fractions of solutions that are real when sampling random Gaussian tensors (with i.i.d. standard normally distributed entries) seem to agree very well with the known theoretical results by Bergqvist and Forrester [13]; they showed that for random Gaussian $n \times n \times 2$ tensors the rank is $n=\frac{\Pi}{\Sigma}$ with probability $p_{n}:=$ $\Gamma\left(\frac{n+1}{2}\right)^{n}(G(n+1))^{-1}$, where $\Gamma$ is the gamma function and $G$ the Barnes $G$-function 
Table 1 Results of sampling GITs in $\sigma_{r ; n_{1}, n_{2}, n_{3}} \subset \mathbb{R}^{n_{1} \times n_{2} \times n_{3}}$ via an acceptance-rejection method

\begin{tabular}{|c|c|c|c|c|c|c|}
\hline \multirow[t]{2}{*}{$n_{1} \times n_{2} \times n_{3}$} & \multirow[t]{2}{*}{$r$} & \multicolumn{3}{|l|}{ Samples } & \multirow[t]{2}{*}{ Fraction in $\mathbb{R}$} & \multirow[t]{2}{*}{ Time (min) } \\
\hline & & $\overline{\mathbb{R}}$ & $\mathbb{C}$ & failed & & \\
\hline $2 \times 2 \times 2$ & 2 & 100,000 & 27,335 & 41 & $\underline{0.7853} \ldots \approx \frac{\pi}{4}$ & 1.3 \\
\hline $3 \times 3 \times 2$ & 3 & 100,000 & 101,345 & 185 & $\underline{0.4966 \ldots \approx \frac{1}{2}}$ & 2.8 \\
\hline $4 \times 4 \times 2$ & 4 & 100,000 & 288,770 & 325 & $\underline{0.2572 \ldots \approx \frac{27 \pi^{2}}{1024}}$ & 14.9 \\
\hline $5 \times 4 \times 3$ & 6 & 100,000 & $1,237,912$ & 643 & $0.0747 \ldots$ & 420.6 \\
\hline $5 \times 5 \times 2$ & 5 & 100,000 & 810,254 & 509 & $\underline{0.1098 \ldots \approx \frac{1}{9}}$ & 99.3 \\
\hline
\end{tabular}

Columns three to five list the number of samples where the final tracked solution of the homotopy was real, complex or failed, respectively. The next column shows the fraction of successful samples that were real; in the case of $n \times n \times 2$ the analytical solution from Bergqvist and Forrester [13] is also stated and the correct digits from the empirical estimate are underlined. The final column indicates the total wall-clock time required to perform the Monte Carlo experiments
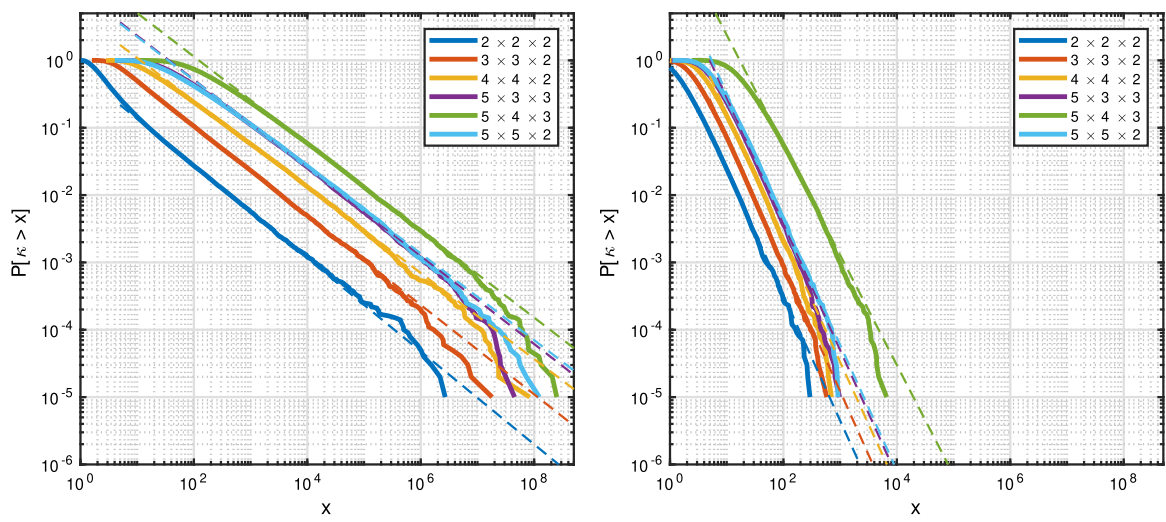

Fig. 1 Empirical complementary cumulative distribution function of the regular and angular condition numbers for the tensor spaces from Table 1. Both plots are on the same scale

(or double gamma function). The correct digits in the numerical approximation are underlined in the penultimate column of Table 1.

The empirical complementary cumulative distribution functions of the regular and angular condition numbers are shown in Fig. 1. The full lines correspond to the empirical data and the thinner dashed lines correspond to an exponential model fitted to the data. From the figure, it is namely reasonable to postulate that the complementary cumulative distribution function $c(x)$ for large $x$ has the form

$$
c(x)=1-\int_{0}^{x} p(t) \mathrm{d} t=a x^{-b}
$$

which corresponds to a straight line in the log-log plot in Fig. 1. We fitted the parameters $a$ and $b$ of the postulated model to the data restricted to the range $10^{-1} \leq c(x) \leq 10^{-3}$. The reason for restricting the data set is that for small condition numbers it is visually evident in Fig. 1 that the model is incorrect and for large condi-

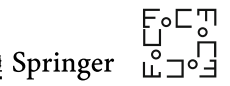


Table 2 Estimated parameters of the exponential model Eq. (37) fitted to the complementary cumulative distribution functions from Fig. 1

\begin{tabular}{lllllll}
\hline$n_{1} \times n_{2} \times n_{3}$ & Regular & \multicolumn{5}{c}{ Angular } \\
\cline { 2 - 7 } & $a$ & $b$ & $R^{2}$ & $a$ & $b$ & $R^{2}$ \\
\hline $2 \times 2 \times 2$ & 0.6624 & 0.6904 & 0.9999 & 1.7288 & 1.8624 & 0.9995 \\
$3 \times 3 \times 2$ & 2.2348 & 0.6636 & 0.9999 & 5.5496 & 1.8856 & 0.9998 \\
$4 \times 4 \times 2$ & 4.7318 & 0.6388 & 0.9997 & 11.4165 & 1.8455 & 0.9994 \\
$5 \times 4 \times 3$ & 22.3141 & 0.6461 & 0.9998 & 102.4887 & 1.6337 & 0.9992 \\
$5 \times 5 \times 2$ & 9.8634 & 0.6436 & 0.9997 & 23.6951 & 1.8662 & 0.9996 \\
\hline
\end{tabular}

The coefficient of determination $R^{2}$ between the log-transformed data and log-transformed model predictions is also indicated

tion numbers the data contain few samples, which negatively impacts the robustness of the fit. The parameters were fitted using fminsearch from MATLAB R2017b with starting point $(1,1)$ and default settings. In all cases, the algorithm terminated because the relative change of the parameters fell below $10^{-4}$. The obtained parameters are shown in Table 2, along with the coefficient of determination $R^{2}$ between the log-transformed data and log-transformed model predictions; 1 indicates perfect correlation.

We may estimate the expected values of the regular and angular condition numbers based on their empirical distributions. If $p(x)$ denotes the probability density function of the regular condition number, then

$$
\mathbb{E} \kappa(\mathcal{A})=\int_{0}^{\infty} x p(x) \mathrm{d} x
$$

From the postulated model of $c(x)$, we find that $p(x)=a b x^{-b-1}$ for large $x$, so that we postulate that the expected value will be well approximated by

$$
\mathbb{E} \kappa(\mathcal{A}) \approx \int_{0}^{\kappa_{0}} x p(x) \mathrm{d} x+a b \int_{\kappa_{0}}^{\infty} x^{-b} \mathrm{~d} x
$$

for some finite $\kappa_{0}$. The expression on the right is finite only if $b>1$. The same discussion applies to the angular condition number as well. Regarding the estimated parameters in Table 2, our empirical data strongly suggest that the expected value of the condition number is infinite for $r=\frac{\Pi}{\Sigma}$ in the tested cases, as $b \approx 0.6<1$. On the other hand, the expected angular condition number seems finite in all cases, as $b \approx 1.8>1$. This suggests that both Theorems 2 and 3 might hold for higher ranks as well.

Acknowledgements We thank the reviewers for helpful suggestions. Part of this work was made while the second and third author were visiting the Universidad de Cantabria, supported by the funds of Grant 21.SI01.64658 (Banco Santander and Universidad de Cantabria), Grant MTM2017-83816-P from the Spanish Ministry of Science. The third author was additionally supported by the FWO Grant for a long stay abroad V401518N. We thank these institutions for their support. 
Funding Open Access funding provided thanks to the CRUE-CSIC agreement with Springer Nature.

Open Access This article is licensed under a Creative Commons Attribution 4.0 International License, which permits use, sharing, adaptation, distribution and reproduction in any medium or format, as long as you give appropriate credit to the original author(s) and the source, provide a link to the Creative Commons licence, and indicate if changes were made. The images or other third party material in this article are included in the article's Creative Commons licence, unless indicated otherwise in a credit line to the material. If material is not included in the article's Creative Commons licence and your intended use is not permitted by statutory regulation or exceeds the permitted use, you will need to obtain permission directly from the copyright holder. To view a copy of this licence, visit http://creativecommons.org/licenses/by/4.0/.

\section{A Proof of the lemmata from Section 3}

\section{A.1 Proof of Lemma 3}

Integrating in polar coordinates, we have

$$
\begin{aligned}
& \int_{(0, \infty)} \int_{(0, \infty)} \lambda^{\Sigma-1} \mu^{\Sigma-1} e^{-\frac{\|\lambda \mathcal{U}+\mu \mathcal{V}\|^{2}}{2}} \mathrm{~d} \lambda \mathrm{d} \mu \\
& =\int_{0}^{\frac{\pi}{2}} \int_{0}^{\infty}(\cos (\theta) \sin (\theta))^{\Sigma-1} \rho^{2 \Sigma-1} e^{-\rho^{2} \frac{\|\cos (\theta) \mathcal{U}+\sin (\theta) \mathcal{V}\|^{2}}{2}} \mathrm{~d} \rho \mathrm{d} \theta .
\end{aligned}
$$

The change of variables $t:=\rho\|\cos (\theta) \mathcal{U}+\sin (\theta) \mathcal{V}\|$ transforms the integral for $\rho$ into

$$
\frac{1}{\|\cos (\theta) \mathcal{U}+\sin (\theta) \mathcal{V}\|^{2 \Sigma}} \int_{0}^{\infty} t^{2 \Sigma-1} e^{-\frac{t^{2}}{2}} \mathrm{~d} t
$$

The last integral is $2^{\Sigma-1} \Gamma(\Sigma)$. Plugging this into the equation above shows the assertion.

\section{A.2 Proof of Lemma 4}

Expanding the denominator and taking a change of variables by $\varphi=2 \theta$, the lemma reduces to proving that

$$
\int_{0}^{\pi / 2} \frac{\cos ^{s-1} \varphi}{(1 \pm a \cos \varphi)^{s}} \mathrm{~d} \varphi=\int_{0}^{\pi / 2} \frac{\sin ^{s-1} \varphi}{(1 \pm a \sin \varphi)^{s}} \mathrm{~d} \varphi \geq \frac{k(s)}{(1 \pm a)^{s-1 / 2}},
$$

(for the first equality, make the change of variables $\varphi \rightarrow \pi / 2-\varphi$ ) where we are denoting $a=|\langle\mathbf{x}, \mathbf{y}\rangle| \in[0,1]$. With the + sign the inequality is clear (choosing an appropriate $k(s)$ ). For the other case, we have, up to a constant $k(s)$, the lower bound

$$
\int_{0}^{\pi / 4} \frac{\mathrm{d} \varphi}{(1-a \cos \varphi)^{s}} \geq \int_{0}^{\pi / 4} \frac{\mathrm{d} \varphi}{\left(1-a\left(1-\varphi^{2} / 2\right)\right)^{s}}=\frac{1}{(1-a)^{s}} \int_{0}^{\pi / 4} \frac{\mathrm{d} \varphi}{\left(1+\frac{a}{1-a} \frac{\varphi^{2}}{2}\right)^{s}} .
$$


Thus, it suffices to check that

$$
\int_{0}^{\pi / 4} \frac{1}{\left(1+\frac{a}{1-a} \frac{\varphi^{2}}{2}\right)^{s}} d \varphi \geq k(s) \sqrt{1-a},
$$

but this is easily checked taking the change of variables $\varphi^{2}=(1-a) t$.

\section{A.3 Proof of Lemma 6}

Recall from the definition of $D(\epsilon)$ that $\left\|\mathbf{u}^{1}-\mathbf{v}^{1}\right\|<\epsilon$, and that $\frac{9}{10}\left\|\mathbf{u}^{1}-\mathbf{v}^{1}\right\|<$ $\left\|\mathbf{u}^{k}-\mathbf{v}^{k}\right\|<\left\|\mathbf{u}^{1}-\mathbf{v}^{1}\right\|$ for $2 \leq k \leq d$. If $\epsilon>0$ is sufficiently small, we can assume

$$
\delta_{k}:=\left\langle\mathbf{u}^{k}, \mathbf{v}^{k}\right\rangle \geq \frac{9}{10}, \quad 1 \leq k \leq d .
$$

To prove the lemma, it suffices to show that

$$
q(U)^{2} \geq 2^{-d}\left(\frac{\left\|\mathbf{u}^{1}-\mathbf{v}^{1}\right\|^{2}}{4}\right)^{\Sigma-1}=2^{-d}\left(\frac{1-\delta_{1}}{2}\right)^{\Sigma-1}
$$

for sufficiently small $\epsilon$.

For convenience, we will first introduce a few auxiliary variables. Consider the next picture:

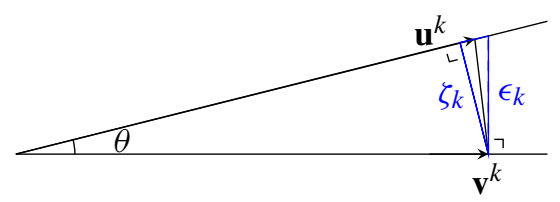

Then, for small $\theta>0$, we have the following elementary trigonometric relations:

$$
\begin{aligned}
& \delta_{k}:=\cos \theta=\left\langle\mathbf{u}^{k}, \mathbf{v}^{k}\right\rangle=\sqrt{\frac{1}{\epsilon_{k}^{2}+1}}, \\
& \zeta_{k}:=\sin \theta=\sqrt{1-\delta_{k}^{2}}=\delta_{k} \epsilon_{k}, \text { and } \\
& \epsilon_{k}:=\tan \theta=\sqrt{\frac{1}{\delta_{k}^{2}}-1 .}
\end{aligned}
$$

It follows from the definition of $D(\epsilon)$ that

$$
\delta_{1}=\min \left\{\delta_{1}, \ldots, \delta_{d}\right\} \quad \text { and } \quad \epsilon_{1}=\max \left\{\epsilon_{1}, \ldots, \epsilon_{d}\right\}
$$


From the previous figure, it is also clear that

$$
\zeta_{k}=\delta_{k} \epsilon_{k} \leq\left\|\mathbf{u}^{k}-\mathbf{v}^{k}\right\| \leq \epsilon_{k}, \quad \text { and so }\left(\frac{9}{10}\right)^{2} \epsilon_{1} \leq \frac{9}{10}\left\|\mathbf{u}^{1}-\mathbf{v}^{1}\right\| \leq\left\|\mathbf{u}^{k}-\mathbf{v}^{k}\right\| \leq \epsilon_{k},
$$

having used in the right sequence of inequalities that Eq. (38) implies $\frac{9}{10} \delta_{1} \epsilon_{1} \geq$ $\left(\frac{9}{10}\right)^{2} \epsilon_{1}$. Then,

$$
1-\frac{\epsilon_{1}^{2}}{2} \leq 1-\frac{\epsilon_{k}^{2}}{2} \leq \delta_{k}=\sqrt{\frac{1}{\epsilon_{k}^{2}+1}} \leq 1-\frac{\epsilon_{k}^{2}}{4} \leq 1-\frac{1}{4}\left(\frac{9}{10}\right)^{4} \epsilon_{1}^{2}
$$

Finally, we will also use

$$
z:=\delta_{1} \cdots \delta_{d}
$$

\section{Transforming $q(U)$}

For computing $q(U)$, we will first make a convenient orthogonal transformation of $U$ 's

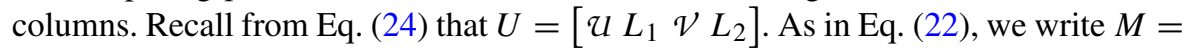
$[\mathcal{U} \mathcal{V}]$. Then, $q(U)=q\left(\left[M L_{1} L_{2}\right]\right)$. The block structure of $L_{1}, L_{2} \in \mathbb{R}^{\Pi \times(\Sigma-1)}$ was given in Eq. (23): $L_{1}$ is made up of the $d$ blocks

$$
L_{1}^{k}=\mathbf{u}^{1} \otimes \cdots \otimes \mathbf{u}^{k-1} \otimes \dot{U}^{k} \otimes \mathbf{u}^{k+1} \otimes \cdots \otimes \mathbf{u}^{d}, \quad 1 \leq k \leq d
$$

and $L_{2}$ is analogously made up of the $d$ blocks

$$
L_{2}^{k}=\mathbf{v}^{1} \otimes \cdots \otimes \mathbf{v}^{k-1} \otimes \dot{V}^{k} \otimes \mathbf{v}^{k+1} \otimes \cdots \otimes \mathbf{v}^{d}, \quad 1 \leq k \leq d,
$$

where $\dot{U}^{k}=\left[\dot{\mathbf{u}}_{2}^{k} \dot{\mathbf{u}}_{3}^{k} \cdots \dot{\mathbf{u}}_{n_{k}}^{k}\right] \in \mathbb{R}^{n_{k} \times n_{k}-1}$ and $\dot{V}^{k}=\left[\dot{\mathbf{v}}_{2}^{k} \dot{\mathbf{v}}_{3}^{k} \cdots \dot{\mathbf{v}}_{n_{k}}^{k}\right] \in \mathbb{R}^{n_{k} \times n_{k}-1}$ are matrices whose columns form an orthonormal basis of $\left(\mathbf{u}^{k}\right)^{\perp}:=\mathrm{T}_{\mathbf{u}^{k}} \mathbb{S}\left(\mathbb{R}^{n_{k}}\right)$ and $\left(\mathbf{v}^{k}\right)^{\perp}:=\mathrm{T}_{\mathbf{v}^{k}} \mathbb{S}\left(\mathbb{R}^{n_{k}}\right)$, respectively.

The columns of $M$ are rotated into

$$
\mathbf{a}_{\uparrow}:=\frac{1}{\sqrt{2}}(\mathcal{U}-\mathcal{V}) \text { and } \mathbf{a}_{\downarrow}:=\frac{1}{\sqrt{2}}(\mathcal{U}+\mathcal{V}),
$$

while the columns of $L_{1}$ and $L_{2}$ are rotated as follows. We define the $\Pi \times(\Sigma-1)$ matrices $R_{\downarrow}:=\left[R_{\downarrow}^{1} \ldots R_{\downarrow}^{d}\right]$ and $R_{\uparrow}:=\left[R_{\uparrow}^{1} \ldots R_{\uparrow}^{d}\right]$, where

$$
R_{\downarrow}^{k}=\frac{1}{\sqrt{2}}\left(L_{1}^{k}-L_{2}^{k}\right) \quad \text { and } \quad R_{\uparrow}^{k}=\frac{1}{\sqrt{2}}\left(L_{1}^{k}+L_{2}^{k}\right) .
$$

The reason for using $\downarrow$ and $\uparrow$ will become clear from the computations below: Inner products of two quantities with arrows pointing in opposite directions are zero, and

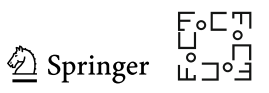


swapping the directions of both arrows flips a sign in the expression of the inner product.

Now, instead of considering the matrix $U$ we work with the matrix

$$
N:=\left[\begin{array}{llll}
\mathbf{a}_{\downarrow} & R_{\downarrow} & \mathbf{a}_{\uparrow} & R_{\uparrow}
\end{array}\right] .
$$

By construction, $\left[\begin{array}{lll}M & L_{1} & L_{2}\end{array}\right]=N Q$ for some orthogonal matrix $Q$, so that

$$
q(U)=q\left(\left[\mathbf{a}_{\downarrow} R_{\downarrow} \mathbf{a}_{\uparrow} R_{\uparrow}\right]\right)=q(N) \quad \text { and } \quad q(U)^{2}=q\left(N^{T} N\right) .
$$

Note that the choice of $\dot{U}^{k}$ and $\dot{V}^{k}$ does not affect the value of $q$. In particular, we may choose the matrices as follows. Let $H$ be the plane in $\mathbb{R}^{n_{k}}$ spanned by $\mathbf{u}^{k}$ and $\mathbf{v}^{k}$. By definition of $D(\epsilon), \mathbf{u}^{k} \neq \pm \mathbf{v}^{k}$. Let $O$ be the rotation that sends $\mathbf{u}^{k}$ to $\mathbf{v}^{k}$, but leaves the orthogonal complement $H^{\perp}$ of $H$ fixed. Take $\dot{\mathbf{u}}_{2}^{k} \in H$ with $\dot{\mathbf{u}}_{2}^{k} \perp \mathbf{u}^{k}$ as the unit norm vector making the smallest angle with $\mathbf{v}^{k}$, as follows:

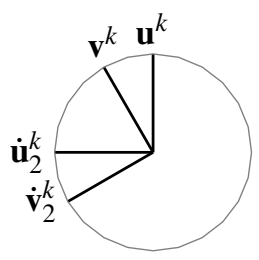

We also take $\dot{\mathbf{v}}_{2}^{k}:=O \dot{\mathbf{u}}_{2}^{k}$, as in the illustration above. If $\mathbf{h}_{3}, \ldots, \mathbf{h}_{n_{k}}$ is any orthogonal basis of $H^{\perp}$, then our choice of bases is

$$
\dot{\mathbf{u}}_{2}^{k} \text { and } \dot{\mathbf{v}}_{2}^{k}=O \dot{\mathbf{u}}_{2}^{k} \text { as above, and for } 3 \leq j \leq n_{k}: \dot{\mathbf{u}}_{j}^{k}=\dot{\mathbf{v}}_{j}^{k}=\mathbf{h}_{j}
$$

In other words, we can assume that all but the first columns of $\dot{U}^{k}$ and $\dot{V}^{k}$ are equal. Moreover, as can be seen from the foregoing figure, the following properties hold:

$$
\begin{aligned}
& \left\langle\dot{\mathbf{u}}_{2}^{k}, \dot{\mathbf{v}}_{2}^{k}\right\rangle=\left\langle Q \mathbf{u}^{k}, Q \mathbf{v}^{k}\right\rangle=\left\langle\mathbf{u}^{k}, \mathbf{v}^{k}\right\rangle=\delta_{k}, \\
& \left\langle\dot{\mathbf{u}}_{2}^{k}, \mathbf{v}^{k}\right\rangle=\cos \left(\frac{\pi}{2}-\arccos \left(\delta_{k}\right)\right)=\sqrt{1-\delta_{k}^{2}}=\delta_{k} \epsilon_{k}, \\
& \left\langle\dot{\mathbf{v}}_{2}^{k}, \mathbf{u}^{k}\right\rangle=\cos \left(\frac{\pi}{2}+\arccos \left(\delta_{k}\right)\right)=-\sqrt{1-\delta_{k}^{2}}=-\delta_{k} \epsilon_{k},
\end{aligned}
$$

where $Q$ is a rotation by $\frac{\pi}{2}$ radians in same direction as the rotation $O$. In particular, we have

$\left(\dot{U}^{k}\right)^{T} \mathbf{v}^{k}=\delta_{k} \epsilon_{k} \mathbf{e}^{k}$ and $\left(\dot{V}^{k}\right)^{T} \mathbf{u}^{k}=-\delta_{k} \epsilon_{k} \mathbf{e}^{k}$, where $\mathbf{e}^{k}:=(1,0, \ldots, 0)^{T} \in \mathbb{R}^{n_{k}-1}$. 
Consider then the Gram matrix $G=N^{T} N$ for this particular choice of tangent vectors:

$$
G=\left[\begin{array}{cccc}
\mathbf{a}_{\downarrow}^{T} \mathbf{a}_{\downarrow} & \mathbf{a}_{\downarrow}^{T} R_{\downarrow} & \mathbf{a}_{\downarrow}^{T} \mathbf{a}_{\uparrow} & \mathbf{a}_{\downarrow}^{T} R_{\uparrow} \\
R_{\downarrow}^{T} \mathbf{a}_{\downarrow} & R_{\downarrow}^{T} R_{\downarrow} & R_{\downarrow}^{T} \mathbf{a}_{\uparrow} & R_{\downarrow}^{T} R_{\uparrow} \\
\mathbf{a}_{\uparrow}^{T} \mathbf{a}_{\downarrow} & \mathbf{a}_{\uparrow}^{T} R_{\downarrow} & \mathbf{a}_{\uparrow}^{T} \mathbf{a}_{\uparrow} & \mathbf{a}_{\uparrow}^{T} R_{\uparrow} \\
R_{\uparrow}^{T} \mathbf{a}_{\downarrow} & R_{\uparrow}^{T} R_{\downarrow} & R_{\uparrow}^{T} \mathbf{a}_{\uparrow} & R_{\uparrow}^{T} R_{\uparrow} .
\end{array}\right] .
$$

We continue by computing its entries.

\section{Inner Products Involving Only a}

Using Eq. (10) for computing inner products of rank-1 tensors, we see that

$$
\mathbf{a}_{\downarrow}^{T} \mathbf{a}_{\downarrow}=1+\langle\mathcal{U}, \mathcal{V}\rangle=1+z, \quad \mathbf{a}_{\downarrow}^{T} \mathbf{a}_{\uparrow}=\mathbf{a}_{\uparrow}^{T} \mathbf{a}_{\downarrow}=0, \text { and } \quad \mathbf{a}_{\uparrow}^{T} \mathbf{a}_{\uparrow}=1-\langle\mathcal{U}, \mathcal{V}\rangle=1-z .
$$

\section{Inner Products Involving both a and $R$}

For each $k$, we have $\left(L_{1}^{k}\right)^{T} \mathcal{U}=\left(L_{2}^{k}\right)^{T} \mathcal{V}=0$,

$$
\left(L_{1}^{k}\right)^{T} \mathcal{V}=\left(\prod_{i \neq k} \delta_{i}\right)\left(\dot{U}^{k}\right)^{T} \mathbf{v}^{k}=z \epsilon_{k} \mathbf{e}^{k} \text { and }\left(L_{2}^{k}\right)^{T} \mathcal{U}=\left(\prod_{i \neq k} \delta_{i}\right)\left(\dot{V}^{k}\right)^{T} \mathbf{u}^{k}=-z \epsilon_{k} \mathbf{e}^{k} .
$$

This implies

$$
\begin{aligned}
& \left(R_{\downarrow}^{k}\right)^{T} \mathbf{a}_{\downarrow}=\frac{1}{2}\left(L_{1}^{k}-L_{2}^{k}\right)^{T}(\mathcal{U}+\mathcal{V})=z \epsilon_{k} \mathbf{e}^{k} . \\
& \left(R_{\downarrow}^{k}\right)^{T} \mathbf{a}_{\uparrow}=\frac{1}{2}\left(L_{1}^{k}-L_{2}^{k}\right)^{T}(\mathcal{U}-\mathcal{V})=0, \\
& \left(R_{\uparrow}^{k}\right)^{T} \mathbf{a}_{\downarrow}=\frac{1}{2}\left(L_{1}^{k}+L_{2}^{k}\right)^{T}(\mathcal{U}+\mathcal{V})=0, \\
& \left(R_{\uparrow}^{k}\right)^{T} \mathbf{a}_{\uparrow}=\frac{1}{2}\left(L_{1}^{k}+L_{2}^{k}\right)^{T}(\mathcal{U}-\mathcal{V})=-z \epsilon_{k} \mathbf{e}^{k},
\end{aligned}
$$

having used Eqs. (46) and (10). Combining the above, we obtain

$$
R_{\uparrow}^{T} \mathbf{a}_{\downarrow}=R_{\downarrow}^{T} \mathbf{a}_{\uparrow}=0, \quad R_{\downarrow}^{T} \mathbf{a}_{\downarrow}=z \mathbf{f}, \quad \text { and } \quad R_{\uparrow}^{T} \mathbf{a}_{\uparrow}=-z \mathbf{f}, \text { where } \mathbf{f}=\left[\begin{array}{c}
\epsilon_{1} \mathbf{e}^{1} \\
\vdots \\
\epsilon_{d} \mathbf{e}^{d}
\end{array}\right]
$$

\section{Inner Products Involving only $R$}

By construction, we have $\left(\dot{U}^{k}\right)^{T} \dot{U}^{k}=\left(\dot{V}^{k}\right)^{T} \dot{V}^{k}=\mathrm{I}_{n_{k}-1}$, where $\mathrm{I}_{n_{k}-1}$ is the $\left(n_{k}-\right.$ $1) \times\left(n_{k}-1\right)$ identity matrix. Furthermore, by our choice of tangent vectors from Eqs.

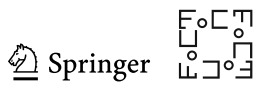


(45) and (46), we have $\left(\dot{U}^{k}\right)^{T} \dot{V}^{k}=\operatorname{diag}\left(\left\langle\dot{\mathbf{u}}_{j}^{k}, \dot{\mathbf{v}}_{j}^{k}\right\rangle\right)_{j=2}^{n_{k}}=\operatorname{diag}\left(\delta_{k}, 1, \ldots, 1\right)$. This implies

$$
\left(L_{1}^{k}\right)^{T} L_{1}^{k}=\left(L_{2}^{k}\right)^{T} L_{2}^{k}=\mathrm{I}_{n_{k}-1}, \quad\left(L_{1}^{k}\right)^{T} L_{2}^{k}=\left(\prod_{i \neq k} \delta_{i}\right)\left(\dot{U}^{k}\right)^{T} \dot{V}^{k}=z \cdot \operatorname{diag}\left(1, \delta_{k}^{-1}, \ldots, \delta_{k}^{-1}\right) .
$$

Moreover, for $j \neq k$ we have

$$
\begin{aligned}
& \left(L_{1}^{j}\right)^{T} L_{1}^{k}=\left(L_{2}^{j}\right)^{T} L_{2}^{k}=0, \\
& \left(L_{1}^{j}\right)^{T} L_{2}^{k}=\left(\prod_{i \notin\{k, j\}} \delta_{i}\right) \cdot\left\{\begin{array}{ll}
\left(\left(\dot{U}^{j}\right)^{T} \mathbf{v}^{j}\right) \otimes\left(\left(\mathbf{u}^{k}\right)^{T} \dot{V}^{k}\right), & j<k, \\
\left(\left(\mathbf{u}^{k}\right)^{T} \dot{V}^{k}\right) \otimes\left(\left(\dot{U}^{j}\right)^{T} \mathbf{v}^{j}\right), & j>k
\end{array}=-z \epsilon_{j} \epsilon_{k} \mathbf{e}^{j}\left(\mathbf{e}^{k}\right)^{T} .\right.
\end{aligned}
$$

From this, we get

$$
\begin{aligned}
& \left(R_{\downarrow}^{j}\right)^{T} R_{\downarrow}^{k}=\frac{1}{2}\left(L_{1}^{j}-L_{2}^{j}\right)^{T}\left(L_{1}^{k}-L_{2}^{k}\right)=\left\{\begin{array}{ll}
\mathrm{I}_{n_{k}-1}-z \cdot \operatorname{diag}\left(1, \delta_{k}^{-1}, \ldots, \delta_{k}^{-1}\right), & j=k \\
z \epsilon_{j} \epsilon_{k} \mathbf{e}^{j}\left(\mathbf{e}^{k}\right)^{T}, & j \neq k
\end{array},\right. \\
& \left(R_{\downarrow}^{j}\right)^{T} R_{\uparrow}^{k}=\frac{1}{2}\left(L_{1}^{j}-L_{2}^{j}\right)^{T}\left(L_{1}^{k}+L_{2}^{k}\right)=0, \\
& \left(R_{\uparrow}^{j}\right)^{T} R_{\uparrow}^{k}=\frac{1}{2}\left(L_{1}^{j}+L_{2}^{j}\right)^{T}\left(L_{1}^{k}+L_{2}^{k}\right)=\left\{\begin{array}{ll}
\mathrm{I}_{n_{k}-1}+z \cdot \operatorname{diag}\left(1, \delta_{k}^{-1}, \ldots, \delta_{k}^{-1}\right), & j=k \\
-z \epsilon_{j} \epsilon_{k} \mathbf{e}^{j}\left(\mathbf{e}^{k}\right)^{T}, & j \neq k
\end{array} .\right.
\end{aligned}
$$

Note that

$$
\mathrm{I}_{n_{k}-1}-z \cdot \operatorname{diag}\left(1, \delta_{k}^{-1}, \ldots, \delta_{k}^{-1}\right)=\mathrm{I}_{n_{k}-1}-z \cdot \operatorname{diag}\left(1+\epsilon_{k}^{2}, \delta_{k}^{-1}, \ldots, \delta_{k}^{-1}\right)+z \epsilon_{k}^{2} \mathbf{e}^{k}\left(\mathbf{e}^{k}\right)^{T},
$$

and

$$
\mathrm{I}_{n_{k}-1}+z \cdot \operatorname{diag}\left(1, \delta_{k}^{-1}, \ldots, \delta_{k}^{-1}\right)=\mathrm{I}_{n_{k}-1}+z \cdot \operatorname{diag}\left(1+\epsilon_{k}^{2}, \delta_{k}^{-1}, \ldots, \delta_{k}^{-1}\right)-z \epsilon_{k}^{2} \mathbf{e}^{k}\left(\mathbf{e}^{k}\right)^{T} .
$$

Exploiting the definition of the vector $\mathbf{f}$ in Eq. (48), the foregoing can be expressed concisely as

$$
R_{\downarrow}^{T} R_{\downarrow}=D_{\downarrow}+z \mathbf{f f}^{T}, \quad R_{\downarrow}^{T} R_{\uparrow}=0, \quad \text { and } \quad R_{\uparrow}^{T} R_{\uparrow}=D_{\uparrow}-z \mathbf{f f}^{T},
$$

where we introduced

$$
\begin{aligned}
& D_{\downarrow}=\mathrm{I}_{\Sigma-1}-z \cdot \operatorname{diag}(1+\epsilon_{1}^{2}, \underbrace{\delta_{1}^{-1}, \ldots, \delta_{1}^{-1}}_{\left(n_{1}-2\right) \text {-times }}, \ldots, 1+\epsilon_{d}^{2}, \underbrace{\delta_{d}^{-1}, \ldots, \delta_{d}^{-1}}_{\left(n_{d}-2\right) \text {-times }}), \text { and } \\
& D_{\uparrow}=\mathrm{I}_{\Sigma-1}+z \cdot \operatorname{diag}(1+\epsilon_{1}^{2}, \underbrace{\delta_{1}^{-1}, \ldots, \delta_{1}^{-1}}_{\left(n_{1}-2\right) \text {-times }}, \ldots, 1+\epsilon_{d}^{2}, \underbrace{\delta_{d}^{-1}, \ldots, \delta_{d}^{-1}}_{\left(n_{d}-2\right) \text {-times }}) .
\end{aligned}
$$




\section{Putting Everything Together}

From the definition of the vector $\mathbf{f}$ in Eq. (48), it is clear that we can construct a permutation matrix $P$ that moves the nonzero elements of $\mathbf{f}$ to the first $d$ positions:

$$
P \mathbf{f}=\left[\begin{array}{l}
\mathbf{g} \\
0
\end{array}\right], \text { where } \mathbf{g}=\left[\epsilon_{1} \cdots \epsilon_{d}\right]^{T}
$$

and 0 is a vector of zeros of length $\Sigma-1-d$. Applying $P^{T}$ on the right of the $R$ 's yields

$$
R_{\downarrow} P^{T}:=\left[T_{\downarrow} S_{\downarrow}\right] \quad \text { and } \quad R_{\uparrow} P^{T}:=\left[T_{\uparrow} S_{\uparrow}\right]
$$

where the $T$ matrices are, respectively, given by

$$
\begin{aligned}
& \sqrt{2} T_{\downarrow}=\left[\mathbf{u}^{1} \otimes \cdots \otimes \mathbf{u}^{k-1} \otimes \dot{\mathbf{u}}_{2}^{k} \otimes \mathbf{u}^{k+1} \otimes \cdots\right. \otimes \mathbf{u}^{d}-\mathbf{v}^{1} \otimes \\
&\left.\cdots \otimes \mathbf{v}^{k-1} \otimes \dot{\mathbf{v}}_{2}^{k} \otimes \mathbf{v}^{k+1} \otimes \cdots \otimes \mathbf{v}^{d}\right]_{k=1}^{d},
\end{aligned}
$$

and analogously for $T_{\uparrow}$ replacing the subtraction by an addition; the matrices $S_{\downarrow}$ and $S_{\uparrow}$ contain the remainder of the columns of $R_{\downarrow}$ and $R_{\uparrow}$, respectively. Then, we have

$$
\begin{aligned}
& P\left(D_{\downarrow}+z \mathbf{f f}^{T}\right) P^{T}=\left[\begin{array}{cc}
E_{\downarrow} & 0 \\
0 & F_{\downarrow}
\end{array}\right]+z\left[\begin{array}{l}
\mathbf{g} \\
0
\end{array}\right]\left[\begin{array}{ll}
\mathbf{g}^{T} & 0^{T}
\end{array}\right]=\left[\begin{array}{cc}
E_{\downarrow}+z \mathbf{g g}^{T} & 0 \\
0 & F_{\downarrow}
\end{array}\right]=\left[\begin{array}{cc}
T_{\downarrow}^{T} T_{\downarrow} & T_{\downarrow}^{T} S_{\downarrow} \\
S_{\downarrow}^{T} T_{\downarrow} & S_{\downarrow}^{T} S_{\downarrow}
\end{array}\right], \text { and } \\
& P\left(D_{\uparrow}-z \mathbf{f f}^{T}\right) P^{T}=\left[\begin{array}{cc}
E_{\uparrow} & 0 \\
0 & F_{\uparrow}
\end{array}\right]-z\left[\begin{array}{l}
\mathbf{g} \\
0
\end{array}\right]\left[\begin{array}{ll}
\mathbf{g}^{T} & 0^{T}
\end{array}\right]=\left[\begin{array}{cc}
E_{\uparrow}-z \mathbf{g g}^{T} & 0 \\
0 & F_{\uparrow}
\end{array}\right]=\left[\begin{array}{cc}
T_{\uparrow}^{T} T_{\uparrow} & T_{\uparrow}^{T} S_{\uparrow} \\
S_{\uparrow}^{T} T_{\uparrow} & S_{\uparrow}^{T} S_{\uparrow}
\end{array}\right],
\end{aligned}
$$

where

$$
\begin{aligned}
& E_{\downarrow}:=\mathrm{I}_{d}-z \cdot \operatorname{diag}\left(1+\epsilon_{1}^{2}, 1+\epsilon_{2}^{2}, \ldots, 1+\epsilon_{d}^{2}\right), \\
& E_{\uparrow}:=\mathrm{I}_{d}+z \cdot \operatorname{diag}(1+\epsilon_{1}^{2}, 1+\epsilon_{2}^{2}, \ldots, 1+\underbrace{\left.\epsilon_{d}^{2}\right),}_{\left(n_{1}-2\right)-\text { times }} \\
& F_{\downarrow}:=\mathrm{I}_{\Sigma-1-d}-z \cdot \operatorname{diag}(\underbrace{\delta_{1}^{-1}, \ldots, \delta_{1}^{-1}}_{\left(n_{d}-2\right) \text {-times }}, \ldots, \underbrace{\delta_{d}^{-1}, \ldots, \delta_{d}^{-1}}_{\left(n_{1}-2\right) \text {-times }}), \text { and } \\
& F_{\uparrow}:=\mathrm{I}_{\Sigma-1-d}+z \cdot \operatorname{diag}(\underbrace{\delta_{1}^{-1}, \ldots, \delta_{1}^{-1}}_{\left(n_{d}-2\right) \text {-times }}, \ldots, \delta_{d}^{-1}, \ldots, \delta_{d}^{-1})
\end{aligned}
$$


Hence, by swapping rows and columns of $G$, which leaves the value of $q$ unchanged because they are orthogonal operations, we find that $q(G)=q\left(G^{\prime}\right)$ with

$$
\begin{aligned}
G^{\prime}:= & {\left[\begin{array}{cccccc}
\mathbf{a}_{\downarrow}^{T} \mathbf{a}_{\downarrow} & \mathbf{a}_{\downarrow}^{T} T_{\downarrow} & \mathbf{a}_{\downarrow}^{T} S_{\downarrow} & \mathbf{a}_{\downarrow}^{T} \mathbf{a}_{\uparrow} & \mathbf{a}_{\downarrow}^{T} T_{\uparrow} & \mathbf{a}_{\downarrow}^{T} S_{\uparrow} \\
T_{\downarrow}^{T} \mathbf{a}_{\downarrow} & T_{\downarrow}^{T} T_{\downarrow} & T_{\downarrow}^{T} S_{\downarrow} & T_{\downarrow}^{T} \mathbf{a}_{\uparrow} & T_{\downarrow}^{T} T_{\uparrow} & T_{\downarrow}^{T} S_{\uparrow} \\
S_{\downarrow}^{T} \mathbf{a}_{\downarrow} & S_{\downarrow}^{T} T_{\downarrow} & S_{\downarrow}^{T} S_{\downarrow} & S_{\downarrow}^{T} \mathbf{a}_{\uparrow} & S_{\downarrow}^{T} T_{\uparrow} & S_{\downarrow}^{T} S_{\uparrow} \\
\mathbf{a}_{\uparrow}^{T} \mathbf{a}_{\downarrow} & \mathbf{a}_{\uparrow}^{T} T_{\downarrow} & \mathbf{a}_{\uparrow}^{T} S_{\downarrow} & \mathbf{a}_{\uparrow}^{T} \mathbf{a}_{\uparrow} & \mathbf{a}_{\uparrow}^{T} T_{\uparrow} & \mathbf{a}_{\uparrow}^{T} S_{\uparrow} \\
T_{\uparrow}^{T} \mathbf{a}_{\downarrow} & T_{\uparrow}^{T} T_{\downarrow} & T_{\uparrow}^{T} S_{\downarrow} & T_{\uparrow}^{T} \mathbf{a}_{\uparrow} & T_{\uparrow}^{T} T_{\uparrow} & T_{\uparrow}^{T} S_{\uparrow} \\
S_{\uparrow}^{T} \mathbf{a}_{\downarrow} & S_{\uparrow}^{T} T_{\downarrow} & S_{\uparrow}^{T} S_{\downarrow} & S_{\uparrow}^{T} \mathbf{a}_{\uparrow} & S_{\uparrow}^{T} T_{\uparrow} & S_{\uparrow}^{T} S_{\uparrow}
\end{array}\right] } \\
= & {\left[\begin{array}{cccccc}
1+z & z \mathbf{g}^{T} & 0 & 0 & 0 & 0 \\
z \mathbf{g} & E_{\downarrow}+z \mathbf{g g}^{T} & 0 & 0 & 0 & 0 \\
0 & 0 & F_{\downarrow} & 0 & 0 & 0 \\
0 & 0 & 0 & 1-z & -z \mathbf{g}^{T} & 0 \\
0 & 0 & 0 & -z \mathbf{g} & E_{\uparrow}-z \mathbf{g g}^{T} & 0 \\
0 & 0 & 0 & 0 & 0 & F_{\uparrow}
\end{array}\right] . }
\end{aligned}
$$

\section{Bounding $q(G)$}

To simplify more, we write

$$
G_{\downarrow}:=\left[\begin{array}{cc}
1 & 0 \\
0 & E_{\downarrow}
\end{array}\right], \quad G_{\uparrow}:=\left[\begin{array}{cc}
1 & 0 \\
0 & E_{\uparrow}
\end{array}\right], \quad \text { and } \quad \mathbf{h}:=\left[\begin{array}{l}
1 \\
\mathbf{g}
\end{array}\right],
$$

so that

$$
q(G)=q\left(G^{\prime}\right)=q\left(\left[\begin{array}{cccc}
G_{\downarrow}+z \mathbf{h h}^{T} & 0 & 0 & 0 \\
0 & G_{\uparrow}-z \mathbf{h h}^{T} & 0 & 0 \\
0 & 0 & F_{\downarrow} & 0 \\
0 & 0 & 0 & F_{\uparrow}
\end{array}\right]\right),
$$

where we swapped some rows and columns again. Because the smallest singular value of the matrix $G_{\uparrow}-z \mathbf{h h}^{T}$ is larger than or equal to the smallest singular value of the positive semidefinite matrix $G^{\prime}$, we obtain the bound

$$
q(G) \geq q\left(G_{\uparrow}-z \mathbf{h} \mathbf{h}^{T}\right) \operatorname{det}\left(G_{\downarrow}+z \mathbf{h} \mathbf{h}^{T}\right) \operatorname{det}\left(F_{\downarrow}\right) \operatorname{det}\left(F_{\uparrow}\right)
$$

We now obtain bounds on the individual factors on the right-hand side of Eq. (52). First, we compute the determinants of the diagonal matrices:

$$
\operatorname{det}\left(F_{\downarrow}\right) \operatorname{det}\left(F_{\uparrow}\right)=\prod_{i=1}^{d}\left(\left(1-z \delta_{i}^{-1}\right)\left(1+z \delta_{i}^{-1}\right)\right)^{n_{i}-2} \geq \prod_{i=1}^{d}\left(1-z \delta_{i}^{-1}\right)^{n_{i}-2},
$$


having used $0<z=\delta_{1} \cdots \delta_{d} \leq 1$. Next, we see that

$$
1-z \delta_{i}^{-1} \geq 1-\delta_{\max }^{d-1} \geq 1-\delta_{\max }>\left(\frac{9}{10}\right)^{2}\left(1-\delta_{1}\right), \text { where } \delta_{\max }:=\max \left\{\delta_{1}, \ldots, \delta_{d}\right\} .
$$

Note that we used $2\left(\frac{9}{10}\right)^{2}\left(1-\delta_{1}\right)=\left(\frac{9}{10}\right)^{2}\left\|\mathbf{u}^{1}-\mathbf{v}^{1}\right\|^{2}<\left\|\mathbf{u}^{k}-\mathbf{v}^{k}\right\|^{2}=2\left(1-\delta_{k}\right)$ in the last inequality. As a result, we obtain

$$
\operatorname{det}\left(F_{\downarrow}\right) \operatorname{det}\left(F_{\uparrow}\right)>\left(\frac{81}{100}\right)^{\Sigma-d-1}\left(1-\delta_{1}\right)^{\Sigma-d-1} \geq\left(\frac{1-\delta_{1}}{2}\right)^{\Sigma-d-1} .
$$

The final determinant in Eq. (52) can be computed as follows. Note that $z \mathbf{h h}^{T}$ is a symmetric matrix with one positive eigenvalue and all others zero. Hence, it follows from Weyl's inequalities [51, Theorem 4.3.7] that the eigenvalues of $G_{\downarrow}$ cannot decrease by adding $z \mathbf{h h}^{T}$. Hence,

$$
\operatorname{det}\left(G_{\downarrow}+z \mathbf{h h}^{T}\right) \geq \operatorname{det}\left(G_{\downarrow}\right)=\operatorname{det}\left(E_{\downarrow}\right)=\prod_{i=1}^{d}\left(1-z\left(1+\epsilon_{i}^{2}\right)\right) .
$$

Next, we bound $1-z\left(1+\epsilon_{i}^{2}\right)$ from below and above. From (40) and (41), we have for some universal constant $C>0$ :

$$
\begin{aligned}
1-z\left(1+\epsilon_{i}^{2}\right) & \leq 1-\left(1-C \epsilon_{1}^{2}\right)^{d+1} \\
& =\left(1-\left(1-C \epsilon_{1}^{2}\right)\right)\left(1+\left(1-C \epsilon_{1}^{2}\right)+\cdots+\left(1-C \epsilon_{1}^{2}\right)^{d}\right) \\
& \leq C(d+1) \epsilon_{1}^{2} .
\end{aligned}
$$

For obtaining the lower bound, note that

$$
\begin{aligned}
z & =\delta_{1} \cdots \delta_{d} \leq \sqrt{\frac{1}{\left(1+\epsilon_{1}^{2}\right)\left(1+\epsilon_{2}^{2}\right)\left(1+\epsilon_{3}^{2}\right)}} \leq \sqrt{\frac{1}{1+\epsilon_{1}^{2}} \frac{1}{\left(1+(9 / 10)^{4} \epsilon_{1}^{2}\right)^{2}}} \\
& =\frac{1}{1+(9 / 10)^{4} \epsilon_{1}^{2}} \sqrt{\frac{1}{1+\epsilon_{1}^{2}}},
\end{aligned}
$$

so that

$$
1-z\left(1+\epsilon_{i}^{2}\right) \geq 1-\frac{\sqrt{1+\epsilon_{1}^{2}}}{1+(9 / 10)^{4} \epsilon_{1}^{2}} \geq \frac{\epsilon_{1}^{2}}{8}
$$

By Eq. (40), $\epsilon_{1}^{2} \geq 2\left(1-\delta_{1}\right)$, so that

$$
\operatorname{det}\left(G_{\downarrow}+z \mathbf{h h}^{T}\right) \geq\left(\frac{2}{8}\right)^{d}\left(1-\delta_{1}\right)^{d}=2^{-d}\left(\frac{1-\delta_{1}}{2}\right)^{d} .
$$

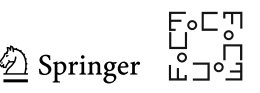


The proof can be completed by a fortunate application of Geršgorin's theorem [43, Satz III]. According to this theorem, the eigenvalues of

$$
G^{\uparrow}-z \mathbf{h h}^{T}=\left[\begin{array}{ccccc}
1-z & -z \epsilon_{1} & -z \epsilon_{2} & \cdots & -z \epsilon_{d} \\
-z \epsilon_{1} & 1+z & -z \epsilon_{1} \epsilon_{2} & \cdots & -z \epsilon_{1} \epsilon_{d} \\
-z \epsilon_{2} & -z \epsilon_{1} \epsilon_{2} & \ddots & & \vdots \\
\vdots & \vdots & & 1+z & -z \epsilon_{d-1} \epsilon_{d} \\
-z \epsilon_{d}-z \epsilon_{1} \epsilon_{d} & \cdots & -z \epsilon_{d-1} \epsilon_{d} & 1+z
\end{array}\right]
$$

are contained in the following Geršgorin disks

$$
\begin{aligned}
\operatorname{disk}_{0} & :=\left\{x \in \mathbb{C}||(1-z)-x \mid \leq z \sum_{k=1}^{d} \epsilon_{k}\right\}, \text { and } \\
\operatorname{disk}_{i} & :=\left\{x \in \mathbb{C}||(1+z)-x \mid \leq z \epsilon_{k}+z \epsilon_{k} \sum_{j \neq k} \epsilon_{j}\right\}, \quad i=1, \ldots, d .
\end{aligned}
$$

Recall from Eq. (42) that $z=\delta_{1} \cdots \delta_{d}$ and from Eq. (39) that $\delta_{k}=\sqrt{\frac{1}{1+\epsilon_{k}^{2}}}$. By choosing $\epsilon=\epsilon_{1}$ small enough, we can get $z$ as close to 1 and $\epsilon_{k} \leq \epsilon_{1}$ as close to 0 as we want. Therefore, if we choose $\epsilon$ small enough, disk $_{0}$ is a small disk near zero, and disk $k_{k}$ are small, pairwise overlapping disks near two. When $\epsilon$ is sufficiently small, disk ${ }_{0}$ is disjoint from the other disks, so that disk $\mathrm{k}_{0}$ contains exactly 1 eigenvalue close to 0 , and $\bigcup_{i=1}^{d} \operatorname{disk}_{i}$ contains $d$ eigenvalues close to 2 . Furthermore, since we are dealing with symmetric matrices, all the eigenvalues are real. Therefore, for sufficiently small $\epsilon$,

$$
q\left(G_{\uparrow}-z \mathbf{h h}^{T}\right) \geq\left(1+z-d \epsilon_{1}\right)^{d} \geq 1 .
$$

Finally, plugging Eqs. (53)-(57) into Eq. (52), we find

$$
q(G) \geq 2^{-d}\left(\frac{1-\delta_{1}}{2}\right)^{\Sigma-1}
$$

This finishes the proof.

\section{B Proof of Lemma 9}

For the proof of Lemma 9, we need the following two simple results. The first one is a well-known result.

Lemma 16 For $1 \leq k \leq d$, let $\mathcal{A}_{k} \subset \mathbb{R}^{n_{k}}$ be a linear subspace of dimension $m_{k}$ and let $\mathcal{A}_{k}^{\perp}$ be its orthogonal complement. Put $\mathcal{V}:=\mathcal{A}_{1} \otimes \cdots \otimes \mathcal{A}_{d}$ and $\mathcal{W}:=\mathcal{A}_{1}^{\perp} \otimes \cdots \otimes \mathcal{A}_{d}^{\perp}$ and let $\mathcal{A} \in \mathcal{S}_{n_{1}, \ldots, n_{d}} \cap \mathcal{V}$ and $\mathcal{B} \in \mathcal{S}_{n_{1}, \ldots, n_{d}} \cap \mathcal{W}$. Then, the tangent spaces $\mathrm{T}_{\mathcal{A}} \mathcal{S}_{n_{1}, \ldots, n_{d}}$ and $\mathrm{T}_{\mathcal{B}} \mathcal{S}_{n_{1}, \ldots, n_{d}}$ are orthogonal to each other. 
Proof Let us write $\mathcal{A}=\mathbf{a}^{1} \otimes \cdots \otimes \mathbf{a}^{d}$ and $\mathcal{B}=\mathbf{b}^{1} \otimes \cdots \otimes \mathbf{b}^{d}$. By Eq. (9), all vectors of the form $\mathbf{a}^{1} \otimes \cdots \otimes \mathbf{a}^{k-1} \otimes \mathbf{v} \otimes \mathbf{a}^{k+1} \otimes \cdots \otimes \mathbf{a}^{d}$ for $1 \leq k \leq d$ and where $\mathbf{v} \in \mathbb{R}^{n_{k}}$ constitute a generating set of $\mathrm{T}_{\mathcal{A}} \mathcal{S}_{n_{1}, \ldots, n_{d}}$. A similar statement holds for $\mathrm{T}_{\mathcal{B}} \mathcal{S}_{n_{1}, \ldots, n_{d}}$. Then, by Eq. (10), the inner product between two such generators $\mathbf{t}:=\mathbf{a}^{1} \otimes \cdots \otimes \mathbf{a}^{k-1} \otimes \mathbf{v} \otimes \mathbf{a}^{k+1} \otimes \cdots \otimes \mathbf{a}^{d}$ and $\mathbf{s}:=\mathbf{b}^{1} \otimes \cdots \otimes \mathbf{b}^{\ell-1} \otimes \mathbf{w} \otimes \mathbf{b}^{\ell+1} \otimes \cdots \otimes \mathbf{b}^{d}$ is

$$
\langle\mathbf{t}, \mathbf{s}\rangle=\left\{\begin{array}{ll}
\langle\mathbf{v}, \mathbf{w}\rangle \prod_{j \neq k}\left\langle\mathbf{a}^{j}, \mathbf{b}^{j}\right\rangle, & \text { if } k=\ell \\
\left\langle\mathbf{a}^{\ell}, \mathbf{w}\right\rangle\left\langle\mathbf{v}, \mathbf{b}^{k}\right\rangle \prod_{j \neq k, \ell}\left\langle\mathbf{a}^{j}, \mathbf{b}^{j}\right\rangle, & \text { if } k \neq \ell
\end{array}=0\right.
$$

because $d \geq 3$ and $\left\langle\mathbf{a}^{j}, \mathbf{b}^{j}\right\rangle=0$ for all $1 \leq j \leq d$. This completes the proof.

Lemma 17 For all $1 \leq k \leq d$, let $\mathcal{A}_{k} \subset \mathbb{R}^{n_{k}}$ be a linear subspace of dimension $m_{i}$, and define the tensor space $\mathcal{V}:=\mathcal{A}_{1} \otimes \cdots \otimes \mathcal{A}_{d}$. Then, the following holds.

1. $\mathcal{S}_{n_{1}, \ldots, n_{d}} \cap \mathcal{V}$ is a manifold.

2. For $1 \leq k \leq d$, let $U_{k} \in \mathbb{R}^{n_{k} \times m_{k}}$ be a matrix whose columns form an orthonormal basis for $\mathcal{A}_{k}$. The map

$$
\mathcal{S}_{m_{1}, \ldots, m_{d}} \rightarrow \mathcal{S}_{n_{1}, \ldots, n_{d}} \cap \mathcal{V}, \mathbf{a}^{1} \otimes \cdots \otimes \mathbf{a}^{d} \mapsto\left(U_{1} \mathbf{a}^{1}\right) \otimes \cdots \otimes\left(U_{d} \mathbf{a}^{d}\right)
$$

is an isometry.

Proof Let us write $U_{1} \otimes \cdots \otimes U_{d}$ for the map from (2). It extends to a linear map $\mathbb{R}^{m_{1}} \otimes \cdots \otimes \mathbb{R}^{m_{d}} \rightarrow \mathbb{R}^{n_{1}} \otimes \cdots \otimes \mathbb{R}^{n_{d}}$ because of the universal property [44, Chapter 1]. By definition, we have $\left(U_{1} \otimes \cdots \otimes U_{d}\right)\left(\mathcal{S}_{m_{1}, \ldots, m_{d}}\right)=\mathcal{S}_{n_{1}, \ldots, n_{d}} \cap \mathcal{V}$ and $\left(U_{1}^{T} \otimes \cdots \otimes\right.$ $\left.U_{d}^{T}\right)\left(\mathcal{S}_{n_{1}, \ldots, n_{d}} \cap \mathcal{V}\right)=\mathcal{S}_{m_{1}, \ldots, m_{d}}$. Hence, $\mathcal{S}_{n_{1}, \ldots, n_{d}} \cap \mathcal{V}$ is the image of a manifold under an invertible linear map. This implies that $\mathcal{S}_{n_{1}, \ldots, n_{d}} \cap \mathcal{V}$ itself is a manifold. Furthermore, $U$ preserves the Euclidean inner product, and so the manifolds $\mathcal{S}_{n_{1}, \ldots, n_{d}} \cap \mathcal{V}$ and $\mathcal{S}_{m_{1}, \ldots, m_{d}}$ are isometric.

We are now ready to prove Lemma 9.

Proof of Lemma 9 We assumed that $\sigma_{r-2 ; n_{1}-2, \ldots, n_{d}-2} \subset \mathbb{R}^{n_{1}-2} \otimes \cdots \otimes \mathbb{R}^{n_{d}-2}$ is generically complex identifiable. Then, Proposition 1(2) tells us that $\mathcal{M}_{r-2 ; n_{1}-2, \ldots, n_{d}-2}$ is Zariski-open in $\left(\mathcal{S}_{n_{1}-2, \ldots, n_{d}-2}\right)^{\times(r-2)}$. Fix any tuple $\left(x_{1}, \ldots, x_{r-2}\right) \in \mathcal{M}_{r-2 ; n_{1}-2, \ldots, n_{d}-2}$. By definition of $\mathcal{M}_{r-2 ; n_{1}-2, \ldots, n_{d}-2}$, the least singular value of the derivative

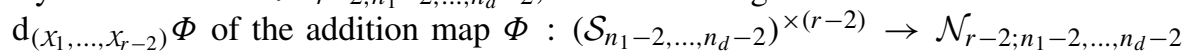
is positive. This implies that $\left|\operatorname{det} \mathrm{d}_{\left(x_{1}, \ldots, x_{r-2}\right)} \Phi\right|>0$. Hence, if $X_{i}$ is a matrix whose columns form an orthonormal basis for $\mathrm{T}_{\mathcal{S}_{n_{1}-2, \ldots, n_{d}-2}} X_{i}$, we have

$$
\left|\operatorname{det} \mathrm{d}_{\left(x_{1}, \ldots, x_{r-2}\right)} \Phi\right|=\operatorname{vol}\left(\left[X_{1} \ldots X_{r-2}\right]\right)>0 \text {. }
$$

Moreover, if we write $x_{i}=v_{i} \mathbf{x}_{i}^{1} \otimes \cdots \otimes \mathbf{x}_{i}^{d}$ with $\left\|\mathbf{x}_{i}^{k}\right\|=1$ and $v_{i}=\left\|X_{i}\right\|$, then we have

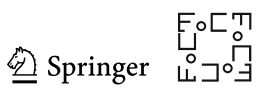

$$
\operatorname{vol}\left[\mathbf{x}_{1}^{h_{1}} \otimes \cdots \otimes \mathbf{x}_{1}^{h_{d-1}} \cdots \mathbf{x}_{r-2}^{h_{1}} \otimes \cdots \otimes \mathbf{x}_{r-2}^{h_{d-1}}\right]>0
$$


for all subsets $\left\{h_{1}, \ldots, h_{d-1}\right\} \subset\{1, \ldots, d\}$ of cardinality $d-1$. Indeed, suppose to the contrary that the foregoing matrix would have linearly dependent columns for some subset; w.l.o.g., we can assume $h_{i}=i$ for $i=1, \ldots, d-1$. Then, there are nonzero $\lambda_{i}$ such that $\sum_{i=1}^{r-2} \lambda_{i} \mathbf{x}_{i}^{1} \otimes \cdots \otimes \mathbf{x}_{i}^{d-1}=0$. Tensoring with an arbitrary vector $\mathbf{v} \in \mathbb{R}^{n_{d}-2}$ yields $\sum_{i=1}^{r-2} \lambda_{i} \mathbf{x}_{i}^{1} \otimes \cdots \otimes \mathbf{x}_{i}^{d-1} \otimes \mathbf{v}=0 \otimes \mathbf{v}=0$, having exploited multilinearity. Note that the $i$ th term in the last sum lives in $\mathrm{T}_{X_{i}} \mathcal{S}_{n_{1}-2, \ldots, n_{d}-2}$ and can be obtained as a linear combination of the columns of the $i$-th block of Terracini's matrix, so that Eq. (12) implies that $\kappa\left(X_{1}, \ldots, X_{r-2}\right)=\infty$, which contradicts $\left(x_{1}, \ldots, x_{r-2}\right) \in$ $\mathcal{M}_{r-2 ; n_{1}-2, \ldots, n_{d}-2}$. We define the following constant, which will play an important role in this proof:

$$
\mu:=\frac{\operatorname{vol}\left(\left[X_{1} \cdots X_{r-2}\right]\right)}{2} \prod_{1 \leq h_{1}<\cdots<h_{d-1} \leq d} \operatorname{vol}\left[\mathbf{x}_{1}^{h_{1}} \otimes \cdots \otimes \mathbf{x}_{1}^{h_{d-1}} \cdots \mathbf{x}_{r-2}^{h_{1}} \otimes \cdots \otimes \mathbf{x}_{r-2}^{h_{d-1}}\right] .
$$

It is important to note that $\mu>0$ is only defined through the choice of $\left(x_{1}, \ldots, x_{r-2}\right)$. The key observation is that we can choose $\mu$ independently of what follows.

Recall that the restriction of $\phi$ to $\mathcal{N}_{2 ; n_{1}, \ldots, n_{d}} \times\left(\mathcal{S}_{n_{1}, \ldots, n_{d}}\right)^{\times(r-2)}$ is

$$
\phi: \mathcal{N}_{2 ; n_{1}, \ldots, n_{d}} \times\left(\mathcal{S}_{n_{1}, \ldots, n_{d}}\right)^{\times r-2} \rightarrow \mathcal{N}_{r}^{*},\left(\mathcal{B}, \mathcal{A}_{1}, \ldots, \mathcal{A}_{r-2}\right) \mapsto \mathcal{B}+\sum_{i=1}^{r-2} \mathcal{A}_{i}
$$

In the terminology of Breiding and Vannieuwenhoven [20], $\mathcal{N}_{r}^{*}$ is the join of $\mathcal{N}_{2 ; n_{1}, \ldots, n_{d}}$ and $r-2$ copies of $\mathcal{S}_{n_{1}, \ldots, n_{d}}$.

Let $\mathcal{B} \in \mathcal{N}_{2 ; n_{1}, \ldots, n_{d}}$. By construction, the tensor $\mathcal{B}$ has multilinear rank bounded by $(2, \ldots, 2)$, meaning that there exists a tensor subspace $\mathcal{V}=\mathcal{A}_{1} \otimes \cdots \otimes \mathcal{A}_{d} \subset$ $\mathbb{R}^{n_{1}} \otimes \cdots \otimes \mathbb{R}^{n_{d}}$ where the linear subspace $\mathcal{A}_{k} \subset \mathbb{R}^{n_{k}}$ has $\operatorname{dim} \mathcal{A}_{k}=2$ and such that $\mathcal{A} \in \mathcal{V}$. For each $1 \leq k \leq d$, we denote the orthogonal complement of $\mathcal{A}_{k}$ in $\mathbb{R}^{n_{k}}$ by $\mathcal{A}_{k}^{\perp}$. Let us define $\mathcal{W}:=\mathcal{A}_{1}^{\perp} \otimes \cdots \otimes \mathcal{A}_{d}^{\perp}$.

Now, we make the following choice of rank-1 tensors $\mathcal{A}_{1}, \ldots, \mathcal{A}_{r-2} \in \mathcal{S}_{n_{1}, \ldots, n_{d}} \cap \mathcal{W}$ : for $1 \leq k \leq d$ let $U_{k} \in \mathbb{R}^{\Pi \times n_{k}-2}$ be a matrix whose columns form an orthonormal basis of $\mathcal{A}_{k}^{\perp}$. Then, by Lemma 17 , the map

$$
U: \mathcal{S}_{n_{1}-2, \ldots, n_{d}-2} \rightarrow \mathcal{S}_{n_{1}, \ldots, n_{d}} \cap \mathcal{W}, \mathbf{a}^{1} \otimes \cdots \otimes \mathbf{a}^{d} \mapsto\left(U_{1} \mathbf{a}^{1}\right) \otimes \cdots \otimes\left(U_{d} \mathbf{a}^{d}\right)
$$

is an isometry of manifolds. For $1 \leq i \leq r-2$, we define $\mathcal{A}_{i}:=U\left(X_{i}\right)$, where $X_{i}$ are the rank-1 tensors from above. We write $\mathcal{A}_{i}=v_{i} \mathbf{a}_{i}^{1} \otimes \cdots \otimes \mathbf{a}_{i}^{d}$. Because $U$ is an isometry, $\left\|X_{i}\right\|=\left\|\mathcal{A}_{i}\right\|=v_{i}$, so we can choose $\left\|\mathbf{a}_{i}^{1}\right\|=\cdots=\left\|\mathbf{a}_{i}^{d}\right\|=1$. The plan for the rest of the proof is to show that $\operatorname{Jac}(\phi)\left(\mathcal{B}, \mathcal{A}_{1}, \ldots, \mathcal{A}_{r-2}\right)$ is a finite value which does not depend on $\mathcal{B}$, and that there is a neighborhood around $\left(\mathcal{A}_{1}, \ldots, \mathcal{A}_{r-2}\right)$, whose size is also independent of $\mathcal{B}$, on which the Jacobian does not deviate too much.

For showing this, we first compute the derivative of $\phi$ at the point $\left(\mathcal{B}, \mathcal{A}_{1}, \ldots, \mathcal{A}_{r-2}\right)$ :

$$
\mathrm{d}_{\left(\mathcal{B}, \mathcal{A}_{1}, \ldots, \mathcal{A}_{r-2}\right)} \phi: \mathrm{T}_{\mathcal{B}} \mathcal{N}_{2 ; n_{1}, \ldots, n_{d}} \times \mathrm{T}_{\mathcal{A}_{1}} \mathcal{S}_{n_{1}, \ldots, n_{d}} \times \cdots \times \mathrm{T}_{\mathcal{A}_{r-2}} \mathcal{S}_{n_{1}, \ldots, n_{d}} \rightarrow \mathrm{T}_{\mathcal{A}} \mathcal{N}_{r}^{*}
$$




$$
\left(\dot{\mathcal{B}}, \dot{\mathcal{A}}_{1}, \ldots, \dot{\mathcal{A}}_{r-2}\right) \mapsto \dot{\mathcal{B}}+\sum_{i=1}^{r-2} \dot{\mathcal{A}}_{i}
$$

where $\mathcal{A}=\mathcal{B}+\sum_{i=1}^{r-2} \mathcal{A}_{i}$. Let $V \in \mathbb{R}^{\Pi \times 2 \Sigma}$ be a matrix whose columns form an orthonormal basis of $\mathrm{T}_{\mathcal{B}} \mathcal{N}_{2 ; n_{1}, \ldots, n_{d}}$, and for $1 \leq i \leq r-2$ let $W_{i} \in \mathbb{R}^{\Pi \times \Sigma}$ be a matrix whose columns form an orthonormal basis of $\mathrm{T}_{\mathcal{A}_{i}} \mathcal{S}_{n_{1}, \ldots, n_{d}}$. Then, we have

$$
\operatorname{Jac}(\phi)\left(\mathcal{B}, \mathcal{A}_{1}, \ldots, \mathcal{A}_{r-2}\right)=\operatorname{vol}\left(\left[V W_{1} \cdots W_{r-2}\right]\right)
$$

Let us write $\mathcal{B}=\mathcal{B}_{1}+\mathcal{B}_{2}$ with $\mathcal{B}_{1}, \mathcal{B}_{2} \in \mathcal{S}_{n_{1}, \ldots, n_{d}}$. From [25, Corollary 2.2], we know that $\mathcal{B}_{1}, \mathcal{B}_{2} \in \mathcal{V}$. Moreover, by Eq. (11), $\mathrm{T}_{\mathcal{B}} \mathcal{N}_{2}=\mathrm{T}_{\mathcal{B}_{1}} \mathcal{S}_{n_{1}, \ldots, n_{d}}+\mathrm{T}_{\mathcal{B}_{2}} \mathcal{S}_{n_{1}, \ldots, n_{d}}$. Since $\mathcal{A}_{1}, \ldots, \mathcal{A}_{r-2}$ are, by assumption, elements of $\mathcal{W}$, Lemma 16 implies that $\mathrm{T}_{\mathcal{B}} \mathcal{N}_{2}$ is orthogonal to $\mathrm{T}_{\mathcal{A}} \mathcal{S}_{n_{1}, \ldots, n_{d}}$ for all $1 \leq i \leq r-2$. Therefore, we get the following equation for Eq. (58):

$$
\operatorname{vol}\left(\left[V W_{1} \cdots W_{r-2}\right]\right)=\operatorname{vol}(V) \operatorname{vol}\left(\left[W_{1} \cdots W_{r-2}\right]\right)=\operatorname{vol}\left(\left[W_{1} \cdots W_{r-2}\right]\right),
$$

the last equality because $V$ has orthonormal columns. Let us further investigate the $W_{i}$.

By Eq. (9), the tangent space of $\mathcal{S}_{n_{1}, \ldots, n_{d}}$ at $\mathcal{A}_{i}=\mathbf{a}_{i}^{1} \otimes \cdots \otimes \mathbf{a}_{i}^{d}$ is

$$
\mathrm{T}_{\mathscr{A}_{i}} \mathcal{S}_{n_{1}, \ldots, n_{d}}=\mathbb{R}^{n_{1}} \otimes \mathbf{a}_{i}^{2} \otimes \cdots \otimes \mathbf{a}_{i}^{d}+\cdots+\mathbf{a}_{i}^{1} \otimes \cdots \otimes \mathbf{a}_{i}^{d-1} \otimes \mathbb{R}^{n_{d}} .
$$

Moreover, by Lemma $17, \mathcal{S}_{n_{1}, \ldots, n_{d}} \cap \mathcal{W}$ is a manifold, and its tangent space at $\mathcal{A}_{i}$ is

$$
\mathrm{T}_{\mathcal{A}_{i}}\left(\mathcal{S}_{n_{1}, \ldots, n_{d}} \cap \mathcal{W}\right)=\mathcal{A}_{1}^{\perp} \otimes \mathbf{a}_{i}^{2} \otimes \cdots \otimes \mathbf{a}_{i}^{d}+\cdots+\mathbf{a}_{i}^{1} \otimes \cdots \otimes \mathbf{a}_{i}^{d-1} \otimes \mathcal{A}_{d}^{\perp} .
$$

For all $1 \leq k \leq d$, let $\left\{\mathbf{t}^{k}, \mathbf{s}^{k}\right\}$ be an orthonormal basis of $\mathcal{A}_{k}$, and let us write

$$
\begin{aligned}
\mathcal{K}_{i} & :=\operatorname{span}\left\{\mathbf{t}^{1} \otimes \mathbf{a}_{i}^{2} \otimes \cdots \otimes \mathbf{a}_{i}^{d}, \ldots, \mathbf{a}_{i}^{1} \otimes \cdots \otimes \mathbf{a}_{i}^{d-1} \otimes \mathbf{t}^{d}\right\}, \text { and } \\
\mathcal{L}_{i} & :=\operatorname{span}\left\{\mathbf{s}^{1} \otimes \mathbf{a}_{i}^{2} \otimes \cdots \otimes \mathbf{a}_{i}^{d}, \ldots, \mathbf{a}_{i}^{1} \otimes \cdots \otimes \mathbf{a}_{i}^{d-1} \otimes \mathbf{s}^{d}\right\} .
\end{aligned}
$$

Because $\mathbf{a}_{i}^{k} \in \mathcal{A}_{k}^{\perp}$ for $1 \leq i \leq r-2$, and because $\left\|\mathbf{a}_{i}^{j}\right\|=1$, the tensors listed even form orthogonal bases for $\mathcal{K}_{i}$ and $\mathcal{L}_{i}$, respectively. Furthermore, we have for all pairs of indices $i, j$ that

$$
\mathrm{T}_{\mathcal{A}_{i}}\left(\mathcal{S}_{n_{1}, \ldots, n_{d}} \cap \mathcal{W}\right) \perp \mathcal{K}_{j}, \quad \mathrm{~T}_{\mathcal{A}_{i}}\left(\mathcal{S}_{n_{1}, \ldots, n_{d}} \cap \mathcal{W}\right) \perp \mathcal{L}_{j}, \quad \text { and } \quad \mathcal{K}_{i} \perp \mathcal{L}_{j}
$$

Therefore, we have the following orthogonal decomposition:

$$
\mathrm{T}_{\mathcal{A}_{i}} \mathcal{S}_{n_{1}, \ldots, n_{d}}=\mathrm{T}_{\mathcal{A}_{i}}\left(\mathcal{S}_{n_{1}, \ldots, n_{d}} \cap \mathcal{W}\right) \oplus \mathcal{K}_{i} \oplus \mathcal{L}_{i}, \text { for } 1 \leq i \leq r-2
$$

The columns of $U X_{i}$ form an orthonormal basis of $\mathrm{T}_{\mathcal{A}_{i}}\left(\mathcal{S}_{n_{1}, \ldots, n_{d}} \cap \mathcal{W}\right)$. Altogether, we have

$$
\operatorname{vol}\left(\left[W_{1} \cdots W_{r-2}\right]\right)
$$

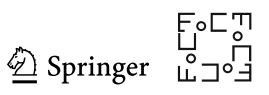




$$
\begin{aligned}
& =\operatorname{vol}\left(\left[U X_{1} \cdots U X_{r-2}\right]\right) \prod \operatorname{vol}\left(\left[\mathbf{a}_{1}^{h_{1}} \otimes \cdots \otimes \mathbf{a}_{1}^{h_{d-1}} \cdots \mathbf{a}_{r-2}^{h_{1}} \otimes \cdots \otimes \mathbf{a}_{r-2}^{h_{d-1}}\right]\right)^{2} \\
& =\operatorname{vol}\left(\left[X_{1} \cdots X_{r-2}\right]\right) \prod \operatorname{vol}\left(\left[\mathbf{x}_{1}^{h_{1}} \otimes \cdots \otimes \mathbf{x}_{1}^{h_{d-1}} \cdots \mathbf{x}_{r-2}^{h_{1}} \otimes \cdots \otimes \mathbf{x}_{r-2}^{h_{d-1}}\right]\right)^{2},
\end{aligned}
$$

where both products range for $1 \leq h_{1}<\cdots<h_{d-1} \leq d$. This implies that

$$
\operatorname{vol}\left(\left[W_{1} \cdots W_{r-2}\right]\right)=2 \mu
$$

is independent of $\mathcal{B}$.

The rest of the proof is a variational argument: Let us denote by $\operatorname{Gr}(\Sigma, \Pi)$ the Grassmann manifold of $\Sigma$-dimensional linear spaces in $\mathbb{R}^{\Pi}$. We endow $\operatorname{Gr}(\Sigma, \Pi)$ with the standard Riemannian metric, such that the distance between two spaces is the Euclidean length of the vector of principal angles [14]. Let us denote this distance by $d(\cdot, \cdot)$. Furthermore, let $G: \mathcal{S}_{n_{1}, \ldots, n_{d}} \rightarrow \operatorname{Gr}(\Sigma, \Pi), \mathcal{A} \mapsto \mathrm{T}_{\mathcal{A}} \mathcal{S}_{n_{1}, \ldots, n_{d}}$ be the Gauss map.

From Breiding and Vannieuwenhoven [23, Proposition 4.3], we get for each $1 \leq i \leq r-2$ that $\left\|\mathrm{d}_{\mathcal{A}_{i}} G\right\| \leq \sqrt{\Sigma}$. This means, that for $\epsilon>0$ and any tuple $\left(\mathcal{A}_{1}^{\prime}, \ldots, \mathcal{A}_{r-2}^{\prime}\right) \in \mathcal{S}_{n_{1}, \ldots, n_{d}}$ satisfying $\left\|\mathcal{A}_{i}-\mathcal{A}_{i}^{\prime}\right\|<\epsilon$ for $1 \leq i \leq r-2$, we have $d\left(\mathrm{~T}_{\mathcal{A}_{i}} \mathcal{S}_{n_{1}, \ldots, n_{d}}, \mathrm{~T}_{\mathcal{A}_{i}^{\prime}} \mathcal{S}_{n_{1}, \ldots, n_{d}}\right)<2 \epsilon \sqrt{\Sigma}$ for sufficiently small $\epsilon$. Let $W_{i}^{\prime} \in \mathbb{R}^{\Pi \times \Sigma}$ be a matrix with orthonormal columns that span $\mathrm{T}_{\mathcal{A}_{i}^{\prime}} \mathcal{S}_{n_{1}, \ldots, n_{d}}$. Consequently, there is a constant $K>0$, which depends only on $n_{1}, \ldots, n_{d}$, such that $\left\|W_{i}-W_{i}^{\prime}\right\|<\epsilon K \sqrt{\Sigma}$.

Recall that the columns of $\left[W_{1} \cdots W_{r-2}\right]$ are orthogonal to the columns of $V$. Moreover, $r \Sigma \leq \Pi$, since we have assumed that $\sigma_{r ; n_{1}, \ldots, n_{d}}$ is generically complex identifiable. Hence, there is a matrix $M \in \mathbb{R}^{\Pi \times \Pi}$ with $M V=V$ and $M W_{i}=W_{i}^{\prime}$ for $1 \leq i \leq r-2$ that leaves the orthogonal complement of $V+W_{1}+\cdots+W_{r-2}$ fixed. This implies

$$
\left\|I_{\Pi}-M\right\|_{2}=\left\|\left(I_{\Pi}-M\right)\left[W_{1} \cdots W_{r-2}\right]\right\|_{2} \leq \sum_{i=1}^{r-2}\left\|W_{i}-W_{i}^{\prime}\right\|<\epsilon r K \sqrt{\Sigma},
$$

where $I_{\Pi}$ is the $\Pi \times \Pi$ identity matrix. Therefore, if we choose $\epsilon$ small enough, we may assume $\operatorname{det} M>\frac{1}{2}$. Note that such a choice of $\epsilon$ is independent of $\mathcal{B}$.

Altogether, we have shown that for all tuples $\left(\mathcal{A}_{1}^{\prime}, \ldots, \mathcal{A}_{r-2}^{\prime}\right) \in \mathcal{S}_{n_{1}, \ldots, n_{d}}$ satisfying $\left\|\mathcal{A}_{i}-\mathcal{A}_{i}^{\prime}\right\|<\epsilon$ we have that

$$
\begin{aligned}
\operatorname{Jac}(\phi)\left(\mathcal{B}, \mathcal{A}_{1}^{\prime}, \ldots, \mathcal{A}_{r-2}^{\prime}\right) & =\operatorname{vol}\left(\left[\begin{array}{ll}
V & W_{1}^{\prime} \cdots W_{r-2}^{\prime}
\end{array}\right]\right) \\
& =\operatorname{vol}\left(\left[M V M W_{1} \cdots M W_{r-2}\right]\right) \\
& =|\operatorname{det}(M)| \operatorname{vol}\left(\left[\begin{array}{lll}
V & W_{1} \cdots W_{r-2}
\end{array}\right]\right) \\
& >\frac{1}{2} \operatorname{vol}\left(\left[\begin{array}{ll}
V & W_{1} \cdots W_{r-2}
\end{array}\right]\right) \\
& =\mu,
\end{aligned}
$$

and both $\epsilon$ and $\mu$ have been chosen independent of $\mathcal{B}$. This concludes the proof.

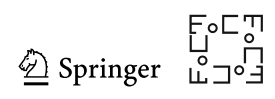




\section{Proofs of the lemmata in Section 5}

\section{C.1 Proof of Lemma 11}

The proof is similar to the proof of Lemma 3. Integrating in polar coordinates, we have

$$
J_{\text {inner }}=\int_{0}^{\frac{\pi}{2}} \int_{0}^{\infty} \rho q\left(\left(\mathrm{I}-M M^{\dagger}\right)\left[\rho \cos (\theta) L_{1} \rho \sin (\theta) L_{2}\right]\right) e^{-\frac{\rho^{2}\|\cos (\theta) u+\sin (\theta) \mathcal{V}\|^{2}}{2}} \mathrm{~d} \rho \mathrm{d} \theta .
$$

Note that the argument of $q$ is a $\Pi \times(2 \Sigma-2)$ matrix. Since $q(A)=\varsigma_{1}(A) \cdots \varsigma_{n-1}(A)$ for $A \in \mathbb{R}^{m \times n}$ with $n \leq m$, we have

$$
q\left(\left(\mathrm{I}-M M^{\dagger}\right)\left[\rho \cos (\theta) L_{1} \rho \sin (\theta) L_{2}\right]\right)=\rho^{2 \Sigma-3} q\left(\left(\mathrm{I}-M M^{\dagger}\right)\left[\cos (\theta) L_{1} \sin (\theta) L_{2}\right]\right)
$$

This yields

$$
J_{\text {inner }}=\int_{0}^{\frac{\pi}{2}} q\left(\left(\mathrm{I}-M M^{\dagger}\right)\left[\cos (\theta) L_{1} \sin (\theta) L_{2}\right]\right) \int_{0}^{\infty} \rho^{2 \Sigma-2} e^{-\frac{\rho^{2}\|\cos (\theta) \mathcal{U}+\sin (\theta) \mathcal{V}\|^{2}}{2}} \mathrm{~d} \rho \mathrm{d} \theta
$$

The change of variables $t=\rho\|\cos (\theta) \mathcal{U}+\sin (\theta) \mathcal{V}\|$ transforms the integral for $\rho$ into

$$
\frac{1}{\|\cos (\theta) \mathcal{U}+\sin (\theta) \mathcal{V}\|^{2 \Sigma-1}} \int_{0}^{\infty} t^{2 \Sigma-2} e^{-\frac{t^{2}}{2}} \mathrm{~d} t=\frac{2^{\frac{2 \Sigma-3}{2}} \Gamma\left(\frac{2 \Sigma-1}{2}\right)}{\|\cos (\theta) \mathcal{U}+\sin (\theta) \mathcal{V}\|^{2 \Sigma-1}}
$$

Plugging the foregoing into the expression for $J_{\text {inner }}$ concludes the proof.

\section{C.2 Proof of Lemma 12}

First, note that the left-hand term in (34) is bounded above by a constant depending on $n_{1}, \ldots, n_{d}, d$. Thus, by choosing an appropriate constant $K$ in (34) we can assume that $\|\mathcal{U}-\mathcal{V}\|$ is smaller than any predefined quantity. We thus assume from now on that $\|\mathcal{U}-\mathcal{V}\| \leq \epsilon$ for some $\epsilon>0$ that can be chosen as small as desired. Furthermore, as in Eq. (39), we write

$$
\delta_{k}:=\left\langle\mathbf{u}^{k}, \mathbf{v}^{k}\right\rangle=\sqrt{\frac{1}{\epsilon_{k}^{2}+1}}, \quad \epsilon_{k}:=\left\|\mathbf{u}^{k}-\mathbf{v}^{k}\right\|=\sqrt{\frac{1}{\delta_{k}^{2}}-1}, \quad \text { and } \quad z:=\delta_{1} \cdots \delta_{d}
$$

We also assume that $\delta_{1}=\min \left\{\delta_{1}, \ldots, \delta_{d}\right\}$, or, equivalently, $\epsilon_{1}=\max \left\{\epsilon_{1}, \ldots, \epsilon_{d}\right\}$. Note that if $\epsilon \approx 0$, then one can assume $\epsilon_{k} \approx 0$ and $\delta_{k} \approx 1$ for all $1 \leq k \leq d$. In particular, all inequalities from the proof of Lemma 6 in Appendix A.3 are still valid here.

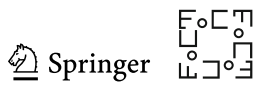




\section{Dropping the Scaling}

For simplifying notation, we abbreviate

$$
A=\left(\mathrm{I}-M M^{\dagger}\right)\left[\begin{array}{ll}
L_{1} & L_{2}
\end{array}\right] \text { and } B=\operatorname{diag}(\underbrace{\cos (\theta), \ldots, \cos (\theta)}_{(\Sigma-1) \text { times }}, \underbrace{\sin (\theta), \ldots, \sin (\theta)}_{(\Sigma-1) \text { times }}) \text {, }
$$

so that $A B=\left(\mathrm{I}-M M^{\dagger}\right)\left[\cos (\theta) L_{1} \sin (\theta) L_{2}\right]$. Observe that, by definition, $A$ ultimately depends on $u$ and $v$; that is, $A=A(u, v)$.

Recall from Eq. (8) that $q(\cdot)$ is the product of all but the smallest singular value of its argument. We first show that $q(A B) \leq q(A)$. To see this, let $\varsigma_{1} \geq \cdots \geq \varsigma_{2}(\Sigma-1) \geq 0$ be the singular values of $A$. Then,

$\operatorname{det}\left((A B)^{T}(A B)\right)=\operatorname{det}\left(B B^{T}\right) \operatorname{det}\left(A^{T} A\right)=\left(\varsigma_{1} \cdots \varsigma_{2}(\Sigma-1)\right)^{2}(\cos (\theta) \sin (\theta))^{2(\Sigma-1)}$.

This shows

$$
q(A B)=\frac{\varsigma_{1} \cdots \varsigma_{2(\Sigma-1)} \cdot(\cos (\theta) \sin (\theta))^{\Sigma-1}}{\varsigma_{\min }(A B)}
$$

where $\varsigma_{\min }(\cdot)$ denotes the smallest singular value; see Eq. (7). Next, we have

$$
\begin{aligned}
\varsigma_{\min }(A B)=\min _{\mathbf{x} \in \mathbb{S}\left(\mathbb{R}^{2(\Sigma-1)}\right)}\|A B \mathbf{x}\| & \geq \min _{\mathbf{x} \in \mathbb{S}\left(\mathbb{R}^{2(\Sigma-1)}\right)}\|A \mathbf{x}\| \cdot \min _{\mathbf{x} \in \mathbb{S}\left(\mathbb{R}^{2(\Sigma-1)}\right)}\|B \mathbf{x}\| \\
& =\varsigma 2(\Sigma-1) \cdot \min \{|\cos (\theta)|,|\sin (\theta)|\} .
\end{aligned}
$$

Moreover, for $0 \leq \theta \leq \frac{\pi}{2}$ we have $0 \leq \cos (\theta) \leq 1$ and $0 \leq \sin (\theta) \leq 1$. Altogether, this implies $q(A \bar{B}) \leq \varsigma_{1} \cdots \varsigma_{2}(\Sigma-1)-1=q(A)$. In the rest of the proof, we bound the $\varsigma_{i}$ 's.

\section{Simplifying the Matrix by Orthogonal Transformations}

Recall from the proof of Lemma 6 that applying the orthogonal transformation in Eq. (44) on the right, we have

$\varsigma_{i}=\varsigma_{i}\left(\left(I-M M^{\dagger}\right)\left[L_{1} L_{2}\right]\right)=\varsigma_{i}\left(\left(I-M M^{\dagger}\right)\left[R_{\uparrow} R_{\downarrow}\right]\right), \quad i=1, \ldots, 2(\Sigma-1)$,

where $\varsigma_{i}(A)$ denotes the $i$ th largest singular value of the matrix $A$. Recalling Eq. (49), we have

$$
\varsigma_{i}=\varsigma_{i}\left((I-P)\left[S_{\uparrow} S_{\downarrow} T_{\uparrow} T_{\downarrow}\right]\right)
$$

where $P=M M^{\dagger}$. Let $\mathbf{a}_{\uparrow}$ and $\mathbf{a}_{\downarrow}$ be as in Eq. (43). The matrix $M M^{\dagger}$ projects orthogonally onto the span of $\mathcal{U}$ and $\mathcal{V}$, which coincides with the span of the orthonormal 
vectors $\frac{\mathbf{a}_{\uparrow}}{\left\|\mathbf{a}_{\uparrow}\right\|}$ and $\frac{\mathbf{a}_{\downarrow}}{\left\|\mathbf{a}_{\downarrow}\right\|}$. Moreover, by Eq. (47) we have $\mathbf{a}_{\downarrow}^{T} \mathbf{a}_{\downarrow}=1+z$ and $\mathbf{a}_{\uparrow}^{T} \mathbf{a}_{\uparrow}=1-z$. This shows that

$$
P=\frac{1}{1+z} \mathbf{a}_{\downarrow} \mathbf{a}_{\downarrow}^{T}+\frac{1}{1-z} \mathbf{a}_{\uparrow} \mathbf{a}_{\uparrow}^{T} .
$$

Next, it follows from Eq. (51) that $P S_{\uparrow}=0$ and $P S_{\downarrow}=0$, so that $\varsigma_{i}=\varsigma_{i}(\tilde{N})$, where

$$
\widetilde{N}:=\left[S_{\uparrow} S_{\downarrow}(I-P) T_{\uparrow}(I-P) T_{\downarrow}\right] .
$$

\section{Computing the Gram Matrix}

Next, we compute the Gram matrix of $\tilde{N}$. Consider again Eq. (51), from which all of the following computations follow. We have

$$
\left[\begin{array}{ll}
S_{\uparrow}^{T} S_{\uparrow} & S_{\uparrow}^{T} S_{\downarrow} \\
S_{\downarrow}^{T} S_{\uparrow} & S_{\downarrow}^{T} S_{\downarrow}
\end{array}\right]=\left[\begin{array}{cc}
F_{\uparrow} & 0 \\
0 & F_{\downarrow}
\end{array}\right],
$$

where the $F$ 's are the diagonal matrices from Eq. (50). From the symmetry of $P$, we obtain $S_{\downarrow}^{T} P=0$ and $S_{\uparrow}^{T} P=0$, so that

$$
\begin{array}{ll}
S_{\uparrow}^{T}(I-P) T_{\uparrow}=S_{\uparrow}^{T} T_{\uparrow}-0=0, & S_{\downarrow}^{T}(I-P) T_{\uparrow}=S_{\downarrow}^{T} T_{\uparrow}-0=0, \\
S_{\uparrow}^{T}(I-P) T_{\downarrow}=S_{\uparrow}^{T} T_{\downarrow}-0=0, & S_{\downarrow}^{T}(I-P) T_{\downarrow}=S_{\downarrow}^{T} T_{\downarrow}-0=0 .
\end{array}
$$

We also find

$$
\begin{aligned}
T_{\uparrow}^{T}(I-P) T_{\downarrow} & =T_{\uparrow}^{T} T_{\downarrow}-T_{\uparrow}^{T}\left(\frac{1}{1+z} \mathbf{a}_{\downarrow} \mathbf{a}_{\downarrow}^{T}+\frac{1}{1-z} \mathbf{a}_{\uparrow} \mathbf{a}_{\uparrow}^{T}\right) T_{\downarrow} \\
& =0-\left(0+\frac{1}{1-z} T_{\uparrow}^{T} \mathbf{a}_{\uparrow} \mathbf{a}_{\uparrow}^{T}\right) T_{\downarrow} \\
& =0 .
\end{aligned}
$$

We observe

$$
\begin{aligned}
T_{\uparrow}^{T}(I-P) T_{\uparrow} & =T_{\uparrow}^{T} T_{\uparrow}-T_{\uparrow}^{T}\left(\frac{1}{1+z} \mathbf{a}_{\downarrow} \mathbf{a}_{\downarrow}^{T}+\frac{1}{1-z} \mathbf{a}_{\uparrow} \mathbf{a}_{\uparrow}^{T}\right) T_{\uparrow} \\
& =\left(E_{\uparrow}-z \mathbf{g g}^{T}\right)-0-\frac{1}{1-z}(-z \mathbf{g})(-z \mathbf{g})^{T} \\
& =E_{\uparrow}-\frac{z}{1-z} \mathbf{g g}^{T} .
\end{aligned}
$$

Analogously, we find

$$
T_{\downarrow}^{T}(I-P) T_{\downarrow}=\left(E_{\downarrow}+z \mathbf{g g}^{T}\right)-\frac{1}{1+z}(z \mathbf{g})(z \mathbf{g})^{T}=E_{\downarrow}+\frac{z}{1+z} \mathbf{g g}^{T} .
$$

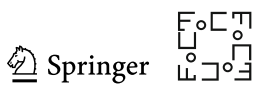


Combining all of the foregoing observations results in

$$
\widetilde{N}^{T} \tilde{N}=\left[\begin{array}{cccc}
F_{\uparrow} & 0 & 0 & 0 \\
0 & F_{\downarrow} & 0 & 0 \\
0 & 0 & E_{\uparrow}-\frac{z}{1-z} \mathbf{g g}^{T} & 0 \\
0 & 0 & 0 & E_{\downarrow}+\frac{z}{1+z} \mathbf{g g}^{T}
\end{array}\right],
$$

so that the singular values of $\widetilde{N}$ are given by the square roots of the eigenvalues of the matrices on the block diagonal.

\section{Bounding the Singular Values}

In the remainder, let $\lambda_{i}(A)$ denote the $i$ th largest eigenvalue of the positive semidefinite matrix $A$. Since $0<\delta_{k} \leq 1$ for all $1 \leq k \leq d$, the eigenvalues $1+z \delta_{k}^{-1}=1+\prod_{j \neq k} \delta_{j}$ for $1 \leq k \leq d$, of the diagonal matrix $F_{\uparrow}$ satisfy

$$
\lambda_{i}\left(F_{\uparrow}\right) \leq 2, \quad i=1, \ldots, \Sigma-d-1
$$

An upper bound for the eigenvalues of $F_{\downarrow}$ is given by

$$
1-z \delta_{k}^{-1}=1-\prod_{1 \leq j \neq k \leq d} \delta_{j} \leq 1-\delta_{1}^{d-1}=\left(1-\delta_{1}\right)\left(1+\delta_{1}+\cdots+\delta_{1}^{d-2}\right) \leq d\left(1-\delta_{1}\right),
$$

since $0<\delta_{1} \leq 1$ for sufficiently small $\epsilon$. Hence, we obtain the bound

$$
\lambda_{i}\left(F_{\downarrow}\right) \leq d\left(1-\delta_{1}\right), \quad i=1, \ldots, \Sigma-d-1 .
$$

The eigenvalues of $E_{\downarrow}+\frac{z}{1+z} \mathbf{g g}^{T}$ can be bounded by using that the spectral norm of the rank-1 term is bounded by $\|\mathbf{g}\|^{2}=\sum_{k=1}^{d} \epsilon_{k}^{2} \leq d \epsilon_{1}^{2}$. It follows from Weyl's perturbation inequality; see, e.g., [51, Corollary 7.3.8], and the positive semidefiniteness of $E_{\downarrow}+\frac{z}{1+z} \mathbf{g g}^{T}$ that

$$
\lambda_{i}\left(E_{\downarrow}+\frac{z}{1+z} \mathbf{g g}^{T}\right) \leq \lambda_{i}\left(E_{\downarrow}\right)+d \epsilon_{1}^{2} .
$$

From the definition of $\delta_{1}$ and $\epsilon_{1}$, we have

$$
\epsilon_{1}^{2} \leq \frac{3}{2}\left\|\mathbf{u}^{1}-\mathbf{v}^{1}\right\|^{2} \leq 3\left(1-\delta_{1}\right)
$$

provided that $\epsilon$ is sufficiently small. The eigenvalues of the diagonal matrix $E_{\downarrow}$ are bounded from above by $C(d+1) \epsilon_{1}^{2}$ due to Eq. (54). Putting all of these together and using $d \geq 1$, we find

$$
\lambda_{i}\left(E_{\downarrow}+\frac{z}{1+z} \mathbf{g g}^{T}\right) \leq C^{\prime} d\left(1-\delta_{1}\right), \quad i=1, \ldots, d,
$$


for some universal constant $C^{\prime}>0$.

For computing an upper bound on the eigenvalues of $E_{\uparrow}-\frac{z}{1-z} \mathbf{g g}^{T}$, we start by noting that

$$
E_{\uparrow}-\frac{z}{1-z} \mathbf{g g}^{T}=-\frac{z}{1-z} \mathbf{g g}^{T}+2 \mathrm{I}_{d}+\operatorname{diag}\left(z\left(1+\epsilon_{1}^{2}\right)-1, \ldots, z\left(1+\epsilon_{d}^{2}\right)-1\right) ;
$$

the spectral norm of the last diagonal matrix is bounded by $C(d+1) \epsilon_{1}^{2}$ because of Eq. (54). Adding the matrix $2 \mathrm{I}_{d}$ causes all eigenvalues to be shifted by 2 ; hence, it suffices to compute the nonzero eigenvalue of the rank-1 matrix. Its eigenvalues are

$$
\underbrace{0, \ldots, 0}_{d-1 \text { times }}, \frac{-z}{1-z}\|\mathbf{g}\|^{2}
$$

the eigenvector corresponding to the last eigenvalue is $\frac{\mathbf{g}}{\|\mathbf{g}\|}$. In order to bound that eigenvalue, note that from (55) and (54) we have for some constant $c=c(d)>0$ :

$$
-z \frac{\|\mathbf{g}\|^{2}}{1-z}=\frac{\sum_{k=1}^{d} \epsilon_{k}^{2}}{1-\prod_{k=1}^{d} \sqrt{\epsilon_{k}^{2}+1}} \leq-2+c \epsilon_{1}^{2}
$$

where the last inequality is proved as follows. Note that $\sqrt{1+t^{2}} \leq 1+\frac{t^{2}}{2}$, which can be verified by squaring the terms and comparing. Then,

$$
\frac{\sum_{k=1}^{d} \epsilon_{k}^{2}}{-1+\prod_{k=1}^{d} \sqrt{\epsilon_{k}^{2}+1}} \geq \frac{\sum_{k=1}^{d} \epsilon_{k}^{2}}{-1+\prod_{k=1}^{d}\left(1+\frac{\epsilon_{k}^{2}}{2}\right)}=\frac{\sum_{k=1}^{d} \epsilon_{k}^{2}}{\frac{1}{2} \sum_{k=1}^{d} \epsilon_{k}^{2}+p},
$$

where $p=p\left(\epsilon_{1}, \ldots, \epsilon_{d}\right)$ is a polynomial expression in the $\epsilon_{i}$ of degree and coefficients bounded by a constant depending only on $d$, with all its monomials of degree at least 4 in $\epsilon_{1}, \ldots, \epsilon_{d}$. Hence, for some constant $c=c(d)$ we have $|p| \leq c(d) \epsilon_{1}^{4}$ and we conclude that

$$
\frac{\sum_{k=1}^{d} \epsilon_{k}^{2}}{-1+\prod_{k=1}^{d} \sqrt{\epsilon_{k}^{2}+1}} \geq \frac{2}{1+\frac{2 c(d) \epsilon_{1}^{4}}{\sum_{k=1}^{d} \epsilon_{k}^{2}}} \geq 2-\frac{4 c(d) \epsilon_{1}^{4}}{\sum_{k=1}^{d} \epsilon_{k}^{2}} \geq 2-\hat{c}(d) \epsilon_{1}^{2},
$$

for some new constant $\hat{c}(d)$; the last step follows from Eq. (40).

Putting all of the foregoing together with Eq. (61), we have thus shown that $E_{\uparrow}-$ $\frac{z}{1-z} \mathbf{g g}^{T}$ has eigenvalues satisfying

$$
\begin{aligned}
& \lambda_{d}\left(E_{\uparrow}-\frac{z}{1-z} \mathbf{g g}^{T}\right) \leq c^{\prime}(d)\left(1-\delta_{1}\right) \text {, and } \\
& \lambda_{i}\left(E_{\uparrow}-\frac{z}{1-z} \mathbf{g g}^{T}\right) \leq 2+c^{\prime}(d)\left(1-\delta_{1}\right), \quad i=1, \ldots, d-1,
\end{aligned}
$$

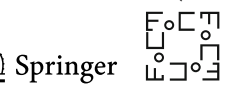


where $c^{\prime}(d)$ is again a constant depending only on $d$.

In Eqs. (59), (60), (62) and (63), we have shown that precisely $\Sigma-d-1+d+1=$ $\Sigma$ eigenvalues are smaller than some constant times $1-\delta_{1}$, while the remaining eigenvalues are clustered near 2 . It follows that there exists a constant $K$, depending only on $n_{1}, \ldots, n_{d}$ and $d$, such that

$$
\begin{aligned}
q\left(\left(I-M M^{\dagger}\right)\left[\cos (\theta) L_{1} \sin (\theta) L_{2}\right]\right) & \leq q\left(\left(I-M M^{\dagger}\right)\left[L_{1} L_{2}\right]\right) \leq K\left(1-\delta_{1}\right)^{\frac{\Sigma-1}{2}} \\
& =K\left(\frac{\left\|\mathbf{u}^{1}-\mathbf{v}^{1}\right\|}{\sqrt{2}}\right)^{\Sigma-1} \leq K\left(\frac{\|\mathcal{U}-\mathcal{V}\|}{\sqrt{2}}\right)^{\Sigma-1}
\end{aligned}
$$

where the last step is by lemma 5. This finishes the proof.

\section{C.3 Proof of Lemma 13}

Let $J$ be the integral in question; i.e.,

$$
J=\int_{0}^{\frac{\pi}{2}} \frac{1}{\|\cos (\theta) \mathbf{x}-\sin (\theta) \mathbf{y}\|^{a}} \mathrm{~d} \theta
$$

Writing $\|\cos (\theta) \mathbf{x}-\sin (\theta) \mathbf{y}\|=\sqrt{1-\sin (2 \theta)\langle\mathbf{x}, \mathbf{y}\rangle}$ and exploiting the symmetry of $\sin (\theta)$ around $\frac{\pi}{4}$, we have

$$
J=2 \int_{0}^{\frac{\pi}{4}} \frac{1}{\sqrt{1-\sin (2 \theta)\langle\mathbf{x}, \mathbf{y}\rangle}^{a}} \mathrm{~d} \theta \leq \int_{0}^{1} \frac{1}{\sqrt{1-(1-t)\langle\mathbf{x}, \mathbf{y}\rangle}^{a} \sqrt{t}} \mathrm{~d} t
$$

the inequality is due to the change of variables $\sin (2 \theta)=1-t$ and $\sqrt{2 t-t^{2}} \geq \sqrt{t}$ for $|t| \leq 1$. Let us write $h:=\langle\mathbf{x}, \mathbf{y}\rangle$. We distinguish between two cases. In the case $h \leq \frac{1}{2}$, we can bound

$$
J \leq \sqrt{2}^{a} \int_{0}^{1} t^{-\frac{1}{2}} \mathrm{~d} t=\sqrt{2}^{a+2} \leq \frac{\sqrt{2}^{3 a}}{\sqrt{2}^{a-1} \sqrt{1-h}^{a-1}}=\frac{\sqrt{2}^{3 a+1}}{\|\mathbf{x}-\mathbf{y}\|^{a-1}}
$$

The second case is $h>\frac{1}{2}$ : A new change of variables $t=\frac{1-h}{h} u$ yields

$$
\begin{aligned}
J & \leq \sqrt{\frac{1-h}{h}} \int_{0}^{\frac{h}{1-h}} \frac{1}{\sqrt{1-h+(1-h) u} a \sqrt{u}} \mathrm{~d} u \\
& =\frac{1}{\sqrt{h}} \frac{1}{\sqrt{1-h}^{a-1}} \int_{0}^{\frac{h}{1-h}} \frac{1}{\sqrt{1+u}^{a} \sqrt{u}} \mathrm{~d} u \\
& \leq \frac{1}{\sqrt{h}} \frac{1}{\sqrt{1-h}^{a-1}}\left(\int_{0}^{1} \frac{1}{\sqrt{1+u} \sqrt{u}} \mathrm{~d} u+\int_{1}^{\infty} \frac{1}{u^{(a+1) / 2}} \mathrm{~d} u\right) .
\end{aligned}
$$


The last integrals add to at most $2+2(a-1)^{-1}$, and we thus have proved for $h>\frac{1}{2}$ :

$$
J \leq \frac{2\left(1+(a-1)^{-1}\right)}{\sqrt{h} \sqrt{1-h}^{a-1}} \leq \frac{\sqrt{2}^{3}\left(1+(a-1)^{-1}\right)}{\sqrt{1-\langle\mathbf{x}, \mathbf{y}\rangle}^{a-1}}=\frac{\sqrt{2}^{a+2}\left(1+(a-1)^{-1}\right)}{\|\mathbf{x}-\mathbf{y}\|^{a-1}} .
$$

The lemma is proved.

\section{C.4 Proof of Lemma 14}

We prove the lemma by induction. The first case $d=1$ reads $\cos \left(\theta_{1}\right) \leq 1-\frac{\theta_{1}^{2}}{7}$. In fact, in this case we have the stronger inequality $\cos (\theta) \leq 1-\frac{\theta^{2}}{4}$ as the following argument shows: Consider the map $f:\left[0, \frac{\pi}{2}\right] \rightarrow \mathbb{R}, \theta \mapsto 1-\frac{\theta^{2}}{4}-\cos (\theta)$. We have $f(0)=0$ and $f\left(\frac{\pi}{2}\right)=1-\frac{\pi^{2}}{16}>0$. Moreover, $f^{\prime}(\theta)=\sin (\theta)-\frac{\theta}{2}>0$ for $0 \leq \theta \leq \frac{\pi}{2}$. This implies that we have $f \geq 0$ proving the case $d=1$. For general $d$, note that by the induction hypothesis

$$
\begin{aligned}
\cos \left(\theta_{1}\right) \cdots \cos \left(\theta_{d}\right) & =\cos \left(\theta_{1}\right) \cdots \cos \left(\theta_{d-1}\right) \cos \left(\theta_{d}\right) \\
& \leq\left(1-\frac{\theta_{1}^{2}+\cdots+\theta_{d-1}^{2}}{7(d-1)}\right) \cos \left(\theta_{d}\right) \\
& \leq\left(1-\frac{\theta_{1}^{2}+\cdots+\theta_{d-1}^{2}}{7 d}\right)\left(1-\frac{\theta_{d}^{2}}{4}\right) \\
& =1-\frac{\theta_{1}^{2}+\cdots+\theta_{d}^{2}}{7 d}+\frac{\theta_{1}^{2}+\cdots+\theta_{d-1}^{2}-7 d+4}{28 d} \theta_{d}^{2} .
\end{aligned}
$$

Since $\theta_{1}^{2}+\cdots+\theta_{d}^{2}-7 d+4 \leq d \frac{\pi^{2}}{4}-7 d+4 \leq 0$ for $d \geq 2$, we conclude the proof of the lemma.

\section{References}

1. Allman ES, Matias C, Rhodes JA (2009) Identifiability of parameters in latent structure models with many observed variables. Ann Statist 37(6A):3099-3132

2. Amelunxen D, Bürgisser P (2015) Probabilistic analysis of the Grassmann condition number. Found Comput Math 15(1):3-51

3. Amelunxen D, Lotz M (2017) Average-case complexity without the black swans. J Complexity 41:82101

4. Anandkumar A, Ge R, Hsu D, Kakade SM, Telgarsky M (2014) Tensor decompositions for learning latent variable models. J Mach Learn Res 15:2773-2832

5. Angelini E, Bocci C, Chiantini L (2017) Real identifiability vs. complex identifiability. Linear Multilinear Algebra 66:1257-1267

6. Armentano D, Beltrán C (2019) The polynomial eigenvalue problem is well conditioned for random inputs. SIAM J Matrix Anal Appl 40(1):175-193

7. Armentano D, Cucker F (2015) A randomized homotopy for the Hermitian eigenpair problem. Found Comput Math 15(1):281-312

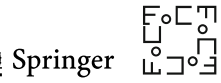


8. Beltrán C, Kozhasov K (2020) The real polynomial eigenvalue problem is well conditioned on the average. Found Comput Math 20(2):291-309

9. Beltrán C, Pardo LM (2011) Fast linear homotopy to find approximate zeros of polynomial systems. Found Comput Math 11(1):95-129

10. Beltrán C, Marzo J, Ortega-Cerdà J (2016) Energy and discrepancy of rotationally invariant determinantal point processes in high dimensional spheres. J Complexity 37:76-109

11. Beltrán C, Breiding P, Vannieuwenhoven N (2019) Pencil-based algorithms for tensor rank decomposition are not stable. SIAM J Matrix Anal Appl 40(2):739-773

12. Benedetti R, Risler JJ (1990) Real algebraic and semi-algebraic sets. Actualités Mathématiques. [Current Mathematical Topics], Hermann, Paris

13. Bergqvist G, Forrester PJ (2011) Rank probabilities for real random $N \times N \times 2$ tensors. Elect Comm in Probab 16:630-637

14. Björck A, Golub GH (1973) Numerical methods for computing angles between linear subspaces. Math Comp 27(123):579-594

15. Blekherman G, Teitler Z (2015) On maximum, typical and generic ranks. Math Ann 362:1021-1031

16. Blum L, Cucker F, Shub M, Smale S (1998) Complexity and Real Computation. Springer-Verlag, New York

17. Bocci C, Chiantini L, Ottaviani G (2014) Refined methods for the identifiability of tensors. Ann Mat Pura Appl 4 193:1691-1702

18. Breiding P, Marigliano O (2020) Random points on an algebraic manifold. SIAM J Math Data Sci 2(3):683-704

19. Breiding P, Timme S (2018) HomotopyContinuation.jl: A package for homotopy continuation in Julia. Mathematical Software - ICMS 2018 Lecture Notes in Computer Science Software available at wwwjuliahomotopycontinuationorg

20. Breiding P, Vannieuwenhoven N (2018) The condition number of join decompositions. SIAM J Matrix Anal Appl 39(1):287-309

21. Breiding P, Vannieuwenhoven N (2018) Convergence analysis of Riemannian Gauss-Newton methods and its connection with the geometric condition number. Appl Math Letters 78:42-50

22. Breiding P, Vannieuwenhoven N (2018) A Riemannian trust region method for the canonical tensor rank approximation problem. SIAM J Optim 28:2435-2465

23. Breiding P, Vannieuwenhoven N (2020) On the average condition number of tensor rank decompositions. IMA J Numer Anal 40(3):1908-1936

24. Breiding P, Vannieuwenhoven N (2021) The condition number of Riemannian approximation problems. SIAM J Optim 31(1):1049-1077

25. Buczynski J J Landsberg (2013) Ranks of tensors and a generalization of secant varieties. Linear Algebra Appl 15:668-689

26. Bürgisser P, Cucker F (2010) Smoothed analysis of Moore-Penrose inversion. SIAM J Matrix Anal Appl 31(5):2769-2783

27. Bürgisser P, Cucker F (2011) On a problem posed by Steve Smale. Ann Math 174:1785-1836

28. Bürgisser P, Cucker F (2013) Condition: The Geometry of Numerical Algorithms, Grundlehren der mathematischen Wissenschaften, vol 349. Springer-Verlag

29. Bürgisser P, Clausen M, Shokrollahi MA (1997) Algebraic Complexity Theory, Grundlehren der mathematischen Wissenshaften, vol 315. Springer, Berlin, Germany

30. Castro D, Montaña JL, Pardo LM, San Martín J (2002) The distribution of condition numbers of rational data of bounded bit length. Found Comput Math 2:1-52

31. Chen Z, Dongarra JJ (2005) Condition numbers of Gaussian random matrices. SIAM J Matrix Anal Appl 27(3):603-620

32. Chiantini L, Ottaviani G (2012) On generic identifiability of 3-tensors of small rank. SIAM J Matrix Anal Appl 33(3):1018-1037

33. Chiantini L, Ottaviani G, Vannieuwenhoven N (2014) An algorithm for generic and low-rank specific identifiability of complex tensors. SIAM J Matrix Anal Appl 35(4):1265-1287

34. Chiantini L, Ottaviani G, Vannieuwenhoven N (2017) Effective criteria for specific identifiability of tensors and forms. SIAM J Matrix Anal Appl 38(2):656-681

35. Comon P (1994) Independent component analysis, a new concept? Signal Proc 36(3):287-314

36. Comon P, Jutten C (2010) Handbook of Blind Source Separation: Independent Component Analysis and Applications. Elsevier 
37. de Silva V, Lim LH (2008) Tensor rank and the ill-posedness of the best low-rank approximation problem. SIAM J Matrix Anal Appl 30(3):1084-1127

38. Demmel J, Diament B, Malajovich G (2001) On the complexity of computing error bounds. Found Comput Math 1:101-125

39. Demmel JW (1988) The probability that a numerical analysis problem is difficult. Math Comp 50:449480

40. Domanov I, De Lathauwer L (2015) Generic uniqueness conditions for the canonical polyadic decomposition and INDSCAL. SIAM J Matrix Anal Appl 36(4):1567-1589

41. Edelman A, Sutton BD (2005) Tails of condition number distributions. SIAM J Matrix Anal Appl 27:547-560

42. Ergür AA, Paouris G, Rojas JM (2019) Probabilistic condition number estimates for real polynomial systems I: A broader family of distributions. Found Comput Math 19(1):131-157

43. Geršgorin S (1931) Über die Abgrenzung der Eigenwerte einer Matrix. Bulletin de l'Académie des Sciences de l'URSS Classe des sciences mathématiques et na (6):749-754

44. Greub WH (1978) Multilinear Algebra. Springer-Verlag

45. Hackbusch W (2012) Tensor Spaces and Numerical Tensor Calculus, Springer Series in Computational Mathematics, vol 42. Springer-Verlag

46. Harris J (1992) Algebraic Geometry, A First Course, Graduate Text in Mathematics, vol 133. SpringerVerlag

47. Hauenstein J, Oeding L, Ottaviani G, Sommese A (2016) Homotopy techniques for tensor decomposition and perfect identifiability. J Reine Angew Math

48. Hauenstein JD, Sottile F (2012) Algorithm 921: alphaCertified: Certifying solutions to polynomial systems. ACM Trans Math Softw 38(28):20

49. Hauser R, Müller T (2009) Conditioning of random conic systems under a general family of input distributions. Found Comput Math 9:335-358

50. Hitchcock FL (1927) The expression of a tensor or a polyadic as a sum of products. J Math Phys 6:164-189

51. Horn R, Johnson C (1990) Matrix Analysis, 2nd edn. Cambridge University Press, New York, NY, USA

52. Howard R (1993) The kinematic formula in Riemannian homogeneous spaces. Mem Amer Math Soc 106(509):vi+69

53. Håstad J (1990) Tensor rank is NP-complete. J Algorithms 11(4):644-654

54. Kroonenberg PM (2008) Applied Multiway Data Analysis. Wiley series in probability and statistics, John Wiley \& Sons, Hoboken, New Jersey

55. Kruskal JB (1977) Three-way arrays: rank and uniqueness of trilinear decompositions, with application to arithmetic complexity and statistics. Linear Algebra Appl 18:95-138

56. Lairez P (2017) A deterministic algorithm to compute approximate roots of polynomial systems in polynomial average time. Found Comput Math 17:1265-1292

57. Landsberg JM (2012) Tensors: Geometry and Applications, Graduate Studies in Mathematics, vol 128. AMS, Providence, Rhode Island

58. Lee JM (2013) Introduction to Smooth Manifolds, Graduate Texts in Mathematics, vol 218, 2nd edn. Springer-Verlag, New York, USA

59. McCullagh P (1987) Tensor Methods in Statistics. Monographs on statistics and applied probability, Chapman and Hall, New York

60. Qi Y, Comon P, Lim LH (2016) Semialgebraic geometry of nonnegative tensor rank. SIAM J Matrix Anal Appl 37:1556-1580

61. Rice JR (1966) A theory of condition. SIAM J Numer Anal 3(2):287-310

62. Shub M, Smale S (1993) Complexity of Bezout's theorem. II. Volumes and probabilities. In: Computational algebraic geometry (Nice, 1992), Progr. Math., vol 109, Birkhäuser Boston, Boston, MA, pp 267-285

63. Shub M, Smale S (1994) Complexity of Bezout's theorem V: polynomial time. Theor Comput Sci 133:141-164

64. Sidiropoulos ND, Giannakis GB, Bro R (2000) Blind PARAFAC receivers for DS-CDMA systems. IEEE Trans Signal Process 48:810-823

65. Sidiropoulos ND, De Lathauwer L, Fu X, Huang K, Papalexakis EE, Faloutsos C (2017) Tensor decomposition for signal processing and machine learning. IEEE Trans Signal Process 65(13):35513582

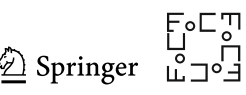


66. Smale S (1981) The fundamental theorem of algebra and complexity theory. Bull Amer Math Soc 4:1-36

67. Smilde A, Bro R, Geladi P (2004) Multi-way Analysis: Applications in the Chemical Sciences. John Wiley \& Sons, Hoboken, New Jersey

68. Spielman DA, Teng SH (2003) Smoothed analysis of termination of linear programming algorithms. vol 97, pp 375-404, iSMP, 2003 (Copenhagen)

69. Trefethen LN, Bau D (1997) Numerical Linear Algebra. SIAM

70. Whitney H (1957) Elementary structure of real algebraic varieties. Ann Math 66(3)

Publisher's Note Springer Nature remains neutral with regard to jurisdictional claims in published maps and institutional affiliations. 